\title{
Nuts and Cardio-Metabolic Disease: A Review of Meta-Analyses
}

\author{
Yoona Kim ${ }^{1}$, Jennifer Keogh ${ }^{2}$ (1) and Peter M. Clifton ${ }^{2, *}$ \\ 1 Department of Food and Nutrition/Institute of Agriculture and Life Science, \\ Gyeongsang National University, Jinju 52828, Korea; yoona.kim@gnu.ac.kr \\ 2 School of Pharmacy and Medical Sciences, University of South Australia, General Post Office Box 2471, \\ Adelaide, SA 5001, Australia; jennifer.keogh@unisa.edu.au \\ * Correspondence: peter.clifton@unisa.edu.au; Tel.: +61-8-8302-1357
}

Received: 13 October 2018; Accepted: 27 November 2018; Published: 6 December 2018

\begin{abstract}
Objectives: Accumulating epidemiological and intervention evidence suggest that nut consumption is associated with reduced incidence of some cardiometabolic diseases. However, to date no review of meta-analyses of epidemiological and intervention studies has evaluated the effects of nut consumption on cardiometabolic disease. Design/Results: Electronic searches for meta-analyses of epidemiological and intervention studies were undertaken in PubMed $^{\circledR} /$ MEDLINE $^{\circledR}$. Meta-analyses of prospective studies show that nut consumption appears to be associated with reduced all-cause mortality by $19-20 \%(n=6)$, cardiovascular disease (CVD) incidence $(19 \% ; n=3)$ and mortality $(25 \% ; n=3)$, coronary heart disease (CHD) incidence (20-34\%; $n=2)$ and mortality $(27-30 \% ; n=2)$ and stroke incidence $(10-11 \% ; n=7)$ and mortality $(18 \%$; $n=2$ ). No association between nut consumption and the risk of type 2 diabetes mellitus (T2DM) was observed in meta-analyses of prospective studies, whereas a decrease in fasting blood glucose ranging from 0.08 to $0.15 \mathrm{mmol} / \mathrm{L}$ was observed in 3 meta-analyses of intervention studies. In the interventions, nut consumption also had favorable effects on total cholesterol $(0.021$ to $0.28 \mathrm{mmol} / \mathrm{L}$ reduction from 8 meta-analyses of interventions) and low-density lipoprotein cholesterol (0.017 to $0.26 \mathrm{mmol} / \mathrm{L}$ reduction from 8 meta-analyses of interventions) and endothelial function ( 0.79 to $1.03 \%$ increase in flow-mediated dilation from 4 meta-analyses of interventions). Nut consumption did not significantly affect body weight. Nut consumption had no effect on inflammatory markers in intervention studies. The effect on blood pressure was inconsistent. A higher nut consumption was associated with a lower incidence of hypertension in prospective studies, while nut consumption did not improve blood pressure in intervention studies. Conclusions: Nut consumption appeared to be associated with lower all-cause mortality and CVD and CHD mortality. There was no association between nut consumption and the incidence of T2DM although fasting blood glucose is decreased in intervention studies. In intervention studies nuts lower total cholesterol and low-density lipoprotein cholesterol (LDL-C).
\end{abstract}

Keywords: nuts; meta-analyses; type 2 diabetes; cardiovascular disease

\section{Introduction}

Cardiometabolic disease is a combination of disorders such as excessive visceral fat, hypertension, atherogenic dyslipidemia and glucose intolesrance which together result in type 2 diabetes mellitus (T2DM) and cardiovascular disease (CVD) [1].

Nuts have been reported to reduce the risk of T2DM and CVD [2-5]. High levels of unsaturated fatty acids and low levels of saturated fatty acids and bioactive compounds (polyphenols, carotenoids, phytosterols, fiber and minerals) may contribute to cardio-protection through improvement of glycemic 
control, lipid profiles, weight, blood pressure, endothelial function, oxidative status, antioxidant and anti-inflammatory action [6,7].

Diabetes, a key cardiometabolic disease is a global health concern with an expected increase in prevalence from $8.4 \%$ in 2017 to $9.9 \%$ in 2045 [8]. People with T2DM have an increased risk of CVD and up to $80 \%$ of individuals with T2DM die from CVD [9]. For this reason, primary prevention of both T2DM and CVD is vital. A healthy diet is important for the prevention and treatment of cardiometabolic disease such as T2DM and CVD [10,11].

This review aims to provide an update on the impact of nut consumption on preventable cardiometabolic diseases by reviewing meta-analyses of epidemiological studies and intervention trials. The evidence for nuts influencing diabetes risk and glycemic control as well as for nuts reducing strokes and weight is very mixed so this review provides a comprehensive review of these controversial areas.

\section{Methods}

We examined meta-analyses of the effects of nut consumption on cardiometabolic diseases in humans. The literature search was performed on the PubMed ${ }^{\circledR} /$ MEDLINE $^{\circledR}$ (https:/ / www.ncbi.nlm. nih.gov / pubmed/) database restricted to full articles in English up to 29 September 2018. The search terms included meta-analysis combined with nut(s) or tree nut(s) or almond(s) or Brazil nut(s) or cashew nut(s) or hazelnut(s) or macadamia(s) or peanut(s) or pistachio(s) or walnut(s). Reference lists of selected papers were also examined. The titles of articles were initially screened and then abstracts and full-texts of literature were reviewed for final study selection. Inclusion criteria were nuts as outlined above, meta-analysis, all-cause mortality, mortality, incidence, CVD, coronary heart disease (CHD), stroke, T2DM, hypertension, metabolic syndrome, obesity, adiposity, glycemic control, glucose, lipids, blood pressure, inflammatory markers, endothelial function, flow-mediated dilation. Meta-analyses of human studies were included and Non-English articles were excluded. Thirty-four meta-analyses that specifically addressed these inclusion criteria were selected and included in this review. A flow chart of the study screening and selection process is reported in Figure 1. 

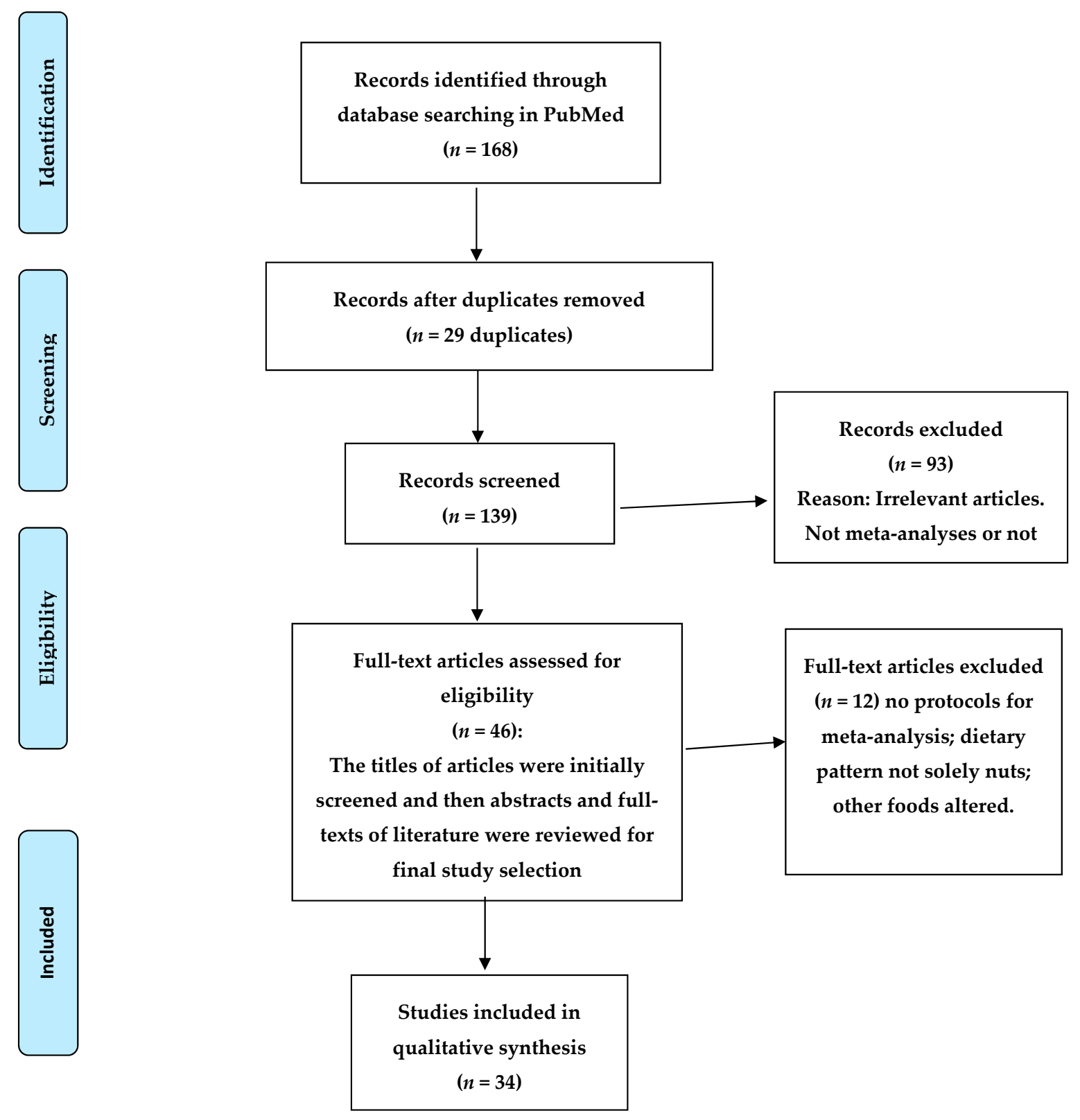

Figure 1. Flow diagram of the literature review.

\section{Results}

\subsection{Prospective Cohort Studies}

All cohort mortality studies are summarized in Table 1 . Forest plots for Table 1 are presented in Supplementary Figures S1-S10.

\subsubsection{All-Cause Mortality}

Meta-analyses [5,12-16] of prospective studies that have examined the effect of nut consumption on all-cause mortality have found a $\sim 19 \%$ reduction in all-cause mortality comparing the highest to the lowest nut intakes. Three meta-analyses $[13,14,16]$ of prospective studies have reported a $\sim 21 \%$ reduction in all-cause mortality per one serving (28 g)/day increase. All meta-analyses show excellent consistency although the number of studies varied in each one.

In the meta-analysis conducted by Chen et al. 2017 [12], nut consumption was related to a reduced risk of all-cause mortality with a relative risk (RR) of 0.81 (95\% confidence interval (CI) $0.78,0.84$; 
$p$-het $=0.20 ; \mathrm{I}^{2}=22 \%$ ) for highest versus lowest consumption in 16 studies (13 publications) [17-29]. Two additional studies $[30,31]$ were included for the dose-response analysis. For risk reduction per serve of nuts non-linear associations ( $p<0.001$ for heterogeneity ( $p$-het)) with all-cause mortality were seen in 18 studies (15 publications) [17-31] (766,470 subjects with 81,034 deaths) with a RR of 0.96 $\left(95 \%\right.$ CI $\left.0.94,0.97 ; \mathrm{I}^{2}=72.3 \%\right)$ per serve of nuts per week. The reduction in mortality levelled off at $\sim 3$ servings/week (12 g/day) of nuts.

Aune et al. 2016 [14] examined 15 studies (15 publications) [17-29,31,32] $(819,448$ subjects with 85,870 deaths)] and showed a RR of 0.81 (95\% CI $0.77,0.85 ; p$-het $=0.05 ; \mathrm{I}^{2}=41 \%$ ) for highest versus lowest nut consumption. Compared with the meta-analysis by Chen et al. 2017 [12], this meta-analysis [14] added 2 more studies [31,32]. A study [32] which appears not to have nuts in the results was included as the authors [14] obtained nut intake information from the first author of the study [32]. Even though the 2 meta-analyses [12,14] had different numbers of studies, subjects and deaths, these showed the same RR of 0.81. Four studies (3 publications) [17-19] (20,2751 subjects with 42,508 deaths) showed a RR of 0.80 (95\% CI 0.74, 0.86; $p$-het $=0.07 ; \mathrm{I}^{2}=58 \%$ ) for tree nuts, while 5 studies ( 3 publications) [17-19] (265,252 subjects with 44,396 deaths) showed a RR of 0.85 $\left(95 \%\right.$ CI $0.82,0.89 ; p$-het $\left.=0.30 ; \mathrm{I}^{2}=18 \%\right)$ for peanuts. In a dose-response analysis, a nonlinear association was observed $(p<0.0001)$ showing no further decrease in risk above $15-20 \mathrm{~g} /$ day of nut consumption. The RR per $28 \mathrm{~g}$ /day in 16 studies (15 publications) [17-29,31,32] was 0.78 (95\% CI 0.72, $0.84 ; p$-het $<0.0001 ; \mathrm{I}^{2}=66 \%$ ).

Other meta-analyses were very similar $[5,12,15,16]$. Schwingshackl et al. 2017 [13] investigated the association between food groups and all-cause mortality in 16 prospective studies [17-19,21-31] with 80,204 deaths. This meta-analysis [13] excluded one study [20] as their analysis [13] excluded populations with chronic disease and studies containing only cause-specific mortality. One serving of nuts $(28 \mathrm{~g})$ / day increase was inversely associated with the risk of all-cause mortality $(\mathrm{RR}=0.76 ; 95 \%$ CI $0.69,0.84 ; \mathrm{I}^{2}=82 \%$; $p$-het $\left.=0.001\right)$ in a nonlinear dose-response manner. The RR was $0.80(95 \% \mathrm{CI}$ $0.74,0.86 ; I^{2}=84 \%$; $p$-het $\left.<0.001\right)$ when comparing the highest with the lowest nut consumption.

Heterogeneity was higher in this analysis [13] but not in Chen et al. 2017 [12]. The higher heterogeneity is explained by between-study differences such as shorter-term follow-up and geographic location (with big differences between European and Asian studies) [13].

\subsubsection{Cause-Specific Mortality}

\section{Diabetes Mortality}

Only one meta-analysis [14] examined the association between nut consumption and diabetes mortality showing a 32\% reduction in diabetes deaths comparing the highest to the lowest nut intakes. It is difficult to know what the nature of a "diabetic death" was the International Classification of Diseases (ICD) code of 250 is very non-specific. The total number of deaths from diabetes were low in each paper. In addition, the study from Luu et al. 2015 was not significant in the original paper whereas it is shown as significant in the meta-analysis casting severe doubt on the conclusions of the meta-analysis.

Aune et al. 2016 [14] found an inverse association between nut consumption and deaths from diabetes in people with T2DM. For highest versus lowest nut consumption, the RR for total nuts from in the meta-analysis containing the studies from Luu et al. 2015 (Southern Community Cohort study and the Shanghai Health study), Bao et al. 2013 (The Nurses' Health Study (NHS)), Bao et al. 2013 (HPFS) and van den Brandt et al. 2015 (Netherlands Cohort study) [17-19] (202,751 subjects with 800 deaths) was $0.68\left(95 \%\right.$ CI $0.52,0.90 ; p$-het $\left.=0.59 ; I^{2}=0 \%\right)$. The RR for peanuts [17-19] $(265,252$ subjects with 901 deaths) was $0.84\left(95 \%\right.$ CI 0.60, 1.19; $p$-het $\left.=0.14 ; I^{2}=42.6 \%\right)$ and the RR for tree $[18,19](130,987$ subjects with 462 deaths) was $1.19\left(95 \%\right.$ CI $0.74,1.89 ; p$-het $\left.=0.43 ; \mathrm{I}^{2}=0 \%\right)$. 


\section{CVD Mortality}

Three meta-analyses $[5,12,15]$ of prospective studies reported a $19-25 \%$ lower rate of CVD mortality comparing highest to lowest consumption.

A recent meta-analysis of prospective studies by Chen et al. 2017 [12] indicated that nut consumption was associated with a lower risk of cardiovascular disease (CVD) mortality. An analysis of highest-versus-lowest consumption showed a $25 \%$ reduction $(R R=0.75 ; 95 \%$ CI $0.71,0.79)$ in CVD mortality from 16 studies (13 publications) [17-23,25-29,33] (524,610 subjects with 19,574 deaths). Tree nuts and peanuts also showed similar reductions with RRs of $0.81(95 \%$ CI $0.74,0.89)$ and 0.78 $(95 \%$ CI $0.73,0.85)$ respectively. Earlier, smaller meta-analyses showed a $26-29 \%$ reduction in CVD in mortality $[5,15]$.

\section{Coronary Heart Disease Mortality}

Chen et al. 2017 [12] found a 27\% reduction ( $R R=0.73 ; 95 \%$ CI 0.67, 0.80; $p$-het $=0.30 ; I^{2}=14.2 \%$ ) in coronary heart disease (CHD) mortality comparing the highest to the lowest intakes of total nuts from 13 studies (10 publications) [17-22,25-28] (429,833 subjects with 10,083 deaths). Tree nuts (21\%) and peanuts $(24 \%)$ also showed similar reductions. Nonlinear inverse associations between nut consumption and cause-specific mortality were found with the greatest decrease at 3 servings / week (12 g/day) $(p<0.001$ for non-linearity), with no further additional reduction in mortality with increasing nut consumption [12]. A similar reduction was found in a smaller study [5].

\section{Stroke Mortality}

A meta-analysis including 12 prospective studies [12] showed an $18 \%$ reduction in stroke mortality while an earlier meta-analysis [5] which included only 3 studies showed a nonsignificant effect.

Chen et al. 2017 [12] showed a lower risk of stroke mortality after nut consumption with a RR of $0.82\left(95 \%\right.$ CI 0.73, 0.91; $p$-het $=0.82 ; \mathrm{I}^{2}=0 \%$ ) from 12 studies (9 publications) $[17-19,21,25,27,28,33,34]$ involving 449,293 subjects with 4398 deaths in a comparison of highest with lowest total nut consumption. On the other hand, a statistically nonsignificant reduction $(R R=0.83 ; 95 \% \mathrm{CI} 0.69,1.00)$ in stroke mortality was observed in analyses of both highest-versus-lowest consumption and dose-response conducted by Mayhew et al. 2016 [5] which only included 4 studies. 
Table 1. Summary of nut consumption and all-cause, CVD, CHD, stroke, diabetes mortality in meta-analyses of prospective cohort studies.

\begin{tabular}{|c|c|c|c|c|c|c|c|c|c|}
\hline & Ref/Studies & Analyses & Nut Types & Sample Size & $\begin{array}{c}\text { SampleAge } \\
\text { (year) }\end{array}$ & $\begin{array}{c}\text { Follow-up } \\
\text { Periods (year) }\end{array}$ & $\begin{array}{l}\text { No. of Studies } \\
\text { Included }\end{array}$ & RRs & Significant? \\
\hline \multirow{11}{*}{$\begin{array}{l}\text { All-cause } \\
\text { mortality }\end{array}$} & \multirow{4}{*}{$\begin{array}{l}\text { Chen et al. } 2017 \\
\text { [12]/18 prospective } \\
\text { studies from } 15 \\
\text { publications [17-31] }\end{array}$} & \multirow{3}{*}{ High- vs. Low } & total nuts & $\begin{array}{c}>498,730 \text { subjects } \\
\text { with } 66,568 \text { deaths }\end{array}$ & $25-85$ & $4.3-30$ & $\begin{array}{l}16 \text { studies from } 13 \\
\text { publications [17-29] }\end{array}$ & $\begin{array}{c}0.81(95 \% \text { CI } 0.78 \\
0.84 ; p \text {-het = 0.20; } \\
\left.I^{2}=22 \%\right)\end{array}$ & $S$ \\
\hline & & & peanuts & $\begin{array}{c}265,252 \text { subjects } \\
\text { with } 44,396 \text { deaths }\end{array}$ & $40-79$ & $1,10 \& 12.2$ & $\begin{array}{c}5 \text { studies from } 3 \\
\text { publications [17-19] }\end{array}$ & $\begin{array}{c}0.85(95 \% \text { CI 0.81; } \\
0.89 ; p \text {-het = 0.19; } \\
\left.\mathrm{I}^{2}=34.1 \%\right)\end{array}$ & $\mathrm{S}$ \\
\hline & & & tree nuts & $\begin{array}{c}130,987 \text { subjects } \\
\text { with } 36,252 \text { deaths }\end{array}$ & $55-69$ & 4,10 & $\begin{array}{c}3 \text { studies from } 2 \\
\text { publications }[17,19]\end{array}$ & $\begin{array}{c}0.83 \text { (95\% CI 0.77, } \\
0.89 ; p \text {-het = 0.95; } \\
\left.\mathrm{I}^{2}=0 \%\right)\end{array}$ & $\mathrm{S}$ \\
\hline & & Dose-response & $\begin{array}{l}\text { per } 1 \text { serving } \\
(28 \mathrm{~g}) \text { of total } \\
\text { nuts per week }\end{array}$ & $\begin{array}{c}>766,470 \text { subjects } \\
\text { with } 81,034 \text { deaths }\end{array}$ & $25-85$ & $4.3-30$ & $\begin{array}{c}18 \text { studies from } 15 \\
\text { publications }[17-31]\end{array}$ & $\begin{array}{c}0.96 \text { (95\% CI 0.94, } \\
0.97 ; p \text {-het < } 0.001 ; \\
\left.I^{2}=72.3 \%\right)\end{array}$ & $\mathrm{S}$ \\
\hline & \multirow{2}{*}{$\begin{array}{c}\text { Schwingshackl et al. } \\
2017[13] / 16 \\
\text { prospective of } 14 \\
\text { publications } \\
{[17-19,21-31]}\end{array}$} & High- vs. Low & total nuts & $\begin{array}{c}902,178 \text { subjects } \\
\text { with } 80,204 \text { deaths }\end{array}$ & $16-87$ & $4.3-30$ & $\begin{array}{c}16 \text { prospective of } 14 \\
\text { publications } \\
{[17-19,21-31]}\end{array}$ & $\begin{array}{c}0.80(95 \% \text { CI } 0.74 \\
0.86 ; I^{2}=84 \% \\
p \text {-het < } 0.001)\end{array}$ & $\mathrm{S}$ \\
\hline & & Dose-response & $\begin{array}{l}\text { per } 1 \text { serving } \\
(28 \mathrm{~g}) / \text { day of } \\
\text { total nuts }\end{array}$ & $\begin{array}{l}902,178 \text { subjects } \\
\text { with } 80,204 \text { deaths }\end{array}$ & $16-87$ & $4.3-30$ & $\begin{array}{c}16 \text { prospective of } 14 \\
\text { publications } \\
{[17-19,21-31]}\end{array}$ & $\begin{array}{c}0.76(95 \% \text { CI } 0.69 \\
0.84 ; I^{2}=82 \% \\
p \text {-het = } 0.001)\end{array}$ & $\mathrm{S}$ \\
\hline & \multirow{5}{*}{$\begin{array}{l}\text { Aune et al. } 2016 \\
{[14] / 20 \text { prospective }} \\
\text { studies from } 9 \\
\text { publications } \\
{[17-29,31-46]}\end{array}$} & \multirow[t]{2}{*}{ High- vs. Low } & peanuts & $\begin{array}{c}265,252 \text { subjects } \\
\text { with } 44,396 \text { deaths }\end{array}$ & $40-79$ & $4-30$ & $\begin{array}{c}5 \text { studies from } 3 \\
\text { publications [17-19] }\end{array}$ & $\begin{array}{c}0.85 \text { (95\% CI 0.82, } \\
0.89 ; p \text {-het = 0.30; } \\
\left.\mathrm{I}^{2}=18 \%\right)\end{array}$ & $\mathrm{S}$ \\
\hline & & & tree nuts & $\begin{array}{c}20,2751 \text { subjects } \\
\text { with } 42,508 \text { deaths }\end{array}$ & $40-79$ & $4-30$ & $\begin{array}{c}4 \text { studies from } 3 \\
\text { publications [17-19] }\end{array}$ & $\begin{array}{c}0.80 \text { (95\% CI 0.74, } \\
0.86 ; p \text {-het = 0.07; } \\
\left.I^{2}=58 \%\right)\end{array}$ & $\mathrm{S}$ \\
\hline & & \multirow{3}{*}{$\begin{array}{l}\text { Dose-response: } \\
28 \mathrm{~g} / \text { day } \\
\text { increase in } \\
\text { total nuts, } \\
10 \mathrm{~g} / \text { day } \\
\text { increase in } \\
\text { peanuts and } \\
\text { tree nuts }\end{array}$} & total nuts & $\begin{array}{c}819,448 \text { subjects } \\
\text { with } 85,870 \text { deaths }\end{array}$ & $25-95$ & $2-30$ & $\begin{array}{c}16 \text { studies from } 15 \\
\text { publications } \\
{[17-29,31,32]}\end{array}$ & $\begin{array}{c}0.78 \text { (95\% CI 0.72, } \\
0.84 ; p \text {-het < } 0.0001 ; \\
\left.\mathrm{I}^{2}=66 \%\right)\end{array}$ & $\mathrm{S}$ \\
\hline & & & peanuts & $\begin{array}{c}265,252 \text { subjects } \\
\text { with } 44,396 \text { deaths }\end{array}$ & $40-79$ & $4-30$ & $\begin{array}{c}5 \text { studies from } 3 \\
\text { publications [17-19] }\end{array}$ & $\begin{array}{c}0.77(95 \% \text { CI } 0.69 \\
0.86 ; p \text {-het = } 0.03 \\
\left.I^{2}=64 \%\right)\end{array}$ & $\mathrm{S}$ \\
\hline & & & tree nuts & $\begin{array}{l}202,751 \text { subjects } \\
\text { with } 42,508 \text { deaths }\end{array}$ & $40-79$ & $4-30$ & $\begin{array}{c}4 \text { studies from } 3 \\
\text { publications [17-19] }\end{array}$ & $\begin{array}{c}0.82(95 \% \text { CI } 0.75 \\
0.90 ; p \text {-het }=0.02 \\
\left.I^{2}=70 \%\right)\end{array}$ & $\mathrm{S}$ \\
\hline
\end{tabular}


Table 1. Cont.

\begin{tabular}{|c|c|c|c|c|c|c|c|c|c|}
\hline & Ref/Studies & Analyses & Nut Types & Sample Size & $\begin{array}{l}\text { SampleAge } \\
\text { (year) }\end{array}$ & $\begin{array}{l}\text { Follow-up } \\
\text { Periods (year) }\end{array}$ & $\begin{array}{l}\text { No. of Studies } \\
\text { Included }\end{array}$ & RRs & Significant? \\
\hline & \multirow{2}{*}{$\begin{array}{c}\text { Mayhew et al. } 2016 \\
\text { [5]/20 Prospective of } \\
20 \text { publications } \\
{[18-20,22-26,33,37-} \\
40,42-44,47-50]\end{array}$} & High- vs. Low & total nuts & $\begin{array}{c}277,432 \text { subjects } \\
\text { with } 49,232 \text { deaths }\end{array}$ & $25-94$ & $4.6-30$ & $\begin{array}{c}10 \text { studies from } 9 \\
\text { publications } \\
{[18,19,22-26,49,50]}\end{array}$ & $\begin{array}{c}0.81(95 \% \text { CI } 0.77 \\
0.85 ; p \text {-het }=0.04 \\
\left.I^{2}=43 \%\right)\end{array}$ & S \\
\hline & & Dose-response & $\begin{array}{l}\text { increase } 4 \\
\text { servings total } \\
\text { nuts/week }\end{array}$ & $\begin{array}{c}277,432 \text { subjects } \\
\text { with } 49,232 \text { deaths }\end{array}$ & $25-94$ & $4.6-30$ & $\begin{array}{c}10 \text { studies from } 9 \\
\text { publications } \\
{[18,19,22-26,49,50]}\end{array}$ & $\begin{array}{c}0.81(95 \% \text { CI } 0.75 \\
0.92)\end{array}$ & S \\
\hline & \multirow{3}{*}{$\begin{array}{c}\text { Grosso et al. } 2015 \\
\text { [15]/9 prospective of } \\
9 \text { publications [19,23- } \\
25,38,39,50-52]\end{array}$} & High- vs. Low & total nuts & $\begin{array}{c}207,608 \text { subjects } \\
\text { with } 34,482 \text { deaths }\end{array}$ & $\geq 18$ & $4.8-30$ & $\begin{array}{c}6 \text { studies from } 6 \\
\text { publications } \\
{[19,23-25,51,52]}\end{array}$ & $\begin{array}{c}0.77(95 \% \text { CI } 0.69 \\
0.87 ; p \text {-het = 0.04; } \\
\left.I^{2}=56 \%\right)\end{array}$ & S \\
\hline & & \multirow{2}{*}{ Dose-response } & $\begin{array}{l}1 \text { serving of } \\
\text { total } \\
\text { nuts/week }\end{array}$ & $\begin{array}{c}263,552 \text { subjects } \\
\text { with } 30,059 \text { deaths }\end{array}$ & $18-80$ & $4.8-30$ & $\begin{array}{c}5 \text { studies from } 5 \\
\text { publications } \\
{[19,23,25,50,51]}\end{array}$ & $\begin{array}{c}0.96(95 \% \text { CI } 0.93 \\
0.98 ; p \text {-het }=0.07 \\
\left.I^{2}=53 \%\right)\end{array}$ & S \\
\hline & & & $\begin{array}{l}1 \text { serving of } \\
\text { total nuts/day }\end{array}$ & $\begin{array}{c}263,552 \text { subjects } \\
\text { with } 30,059 \text { deaths }\end{array}$ & $18-80$ & $4.8-30$ & $\begin{array}{c}5 \text { studies from } 5 \\
\text { publications } \\
{[19,23,25,50,51]}\end{array}$ & $\begin{array}{c}0.73(95 \% \text { CI } 0.60 \\
0.88 ; p \text {-het }=0.07 \\
\left.I^{2}=53 \%\right)\end{array}$ & S \\
\hline & \multirow{2}{*}{$\begin{array}{c}\text { Luo et al. } 2014 \\
\text { [16]/18 prospective } \\
\text { of } 18 \text { publications } \\
{[19,20,22,24-26,38-40} \\
42-44,48,50,53-56]\end{array}$} & $\begin{array}{c}\text { High ( } \geq 2 \text { to } \\
\geq 7 \\
\text { servings/week } \\
\text { vs. Low ( } 0 \text { to } \\
\leq 1 \\
\text { serving/week) }\end{array}$ & total nuts & 48,818 deaths & $25-94$ & $4-30$ & $\begin{array}{c}7 \text { studies from } 7 \\
\text { publications } \\
{[19,20,22,24-26,50]}\end{array}$ & $\begin{array}{c}0.85 \text { (95\% CI 0.79; } \\
0.91 ; p \text {-het = 0.005; } \\
\left.\mathrm{I}^{2}=60.2 \%\right)\end{array}$ & S \\
\hline & & Dose-response & total nuts & $\begin{array}{l}3,112,510 \text { subjects } \\
\text { with } 33,595 \text { deaths }\end{array}$ & $40-80$ & $4-30$ & $\begin{array}{c}4 \text { studies from } 4 \\
\text { publications } \\
{[19,22,25,26]}\end{array}$ & $\begin{array}{c}0.83 \text { (95\% CI 0.76, } \\
0.91 ; p \text {-het }=0.032 ; \\
\left.\mathrm{I}^{2}=62.1 \%\right)\end{array}$ & S \\
\hline \multirow{4}{*}{$\begin{array}{l}\text { CVD } \\
\text { mortality }\end{array}$} & \multirow{4}{*}{$\begin{array}{l}\text { Chen et al. } 2017 \\
\text { [12]/18 prospective } \\
\text { studies from } 15 \\
\text { publications }[17-31]\end{array}$} & \multirow{3}{*}{ High- vs. Low } & total nuts & $\begin{array}{c}>524,610 \text { subjects } \\
\text { with } 19,574 \text { deaths }\end{array}$ & $25-85$ & $4.3-30$ & $\begin{array}{l}16 \text { studies from } 13 \\
\text { publications } \\
{[17-23,25-29,33]}\end{array}$ & $\begin{array}{c}0.75 \text { (95\% CI 0.71, } \\
0.79 ; p \text {-het = 0.50; } \\
\left.I^{2}=0 \%\right)\end{array}$ & S \\
\hline & & & peanuts & $\begin{array}{c}265,252 \text { subjects } \\
\text { with } 12,052 \text { deaths }\end{array}$ & $40-79$ & $1,10 \& 12.2$ & $\begin{array}{c}5 \text { studies from } 3 \\
\text { publications [17-19] }\end{array}$ & $\begin{array}{c}0.78(95 \% \mathrm{CI} \\
0.73-0.85 \\
p \text {-het = 0.30; } \\
\left.\mathrm{I}^{2}=18 \%\right) \\
\end{array}$ & S \\
\hline & & & tree nuts & $\begin{array}{l}130,987 \text { subjects } \\
\text { with } 9456 \text { deaths }\end{array}$ & $55-69$ & 4,10 & $\begin{array}{c}3 \text { studies from } 2 \\
\text { publications }[17,19]\end{array}$ & $\begin{array}{c}0.81(95 \% \text { CI } 0.74 \\
0.89 ; p \text {-het }=0.62 ; \\
\left.I^{2}=0 \%\right)\end{array}$ & S \\
\hline & & Dose-response & $\begin{array}{l}\text { per } 1 \text { serving } \\
\text { of total nuts } \\
\text { per week }\end{array}$ & $\begin{array}{l}>509,871 \text { subjects } \\
\text { with } 20,362 \text { deaths }\end{array}$ & $25-85$ & $4.3-30$ & $\begin{array}{c}16 \text { studies from } 13 \\
\text { publications } \\
{[17-22,25-29,31,33]}\end{array}$ & $\begin{array}{c}0.94 \text { (95\% CI 0.93, } \\
0.96 ; p \text {-het }=0.001 ; \\
\left.\mathrm{I}^{2}=59.9 \%\right)\end{array}$ & S \\
\hline
\end{tabular}


Table 1. Cont

\begin{tabular}{|c|c|c|c|c|c|c|c|c|c|}
\hline & Ref/Studies & Analyses & Nut Types & Sample Size & $\begin{array}{c}\text { SampleAge } \\
\text { (year) }\end{array}$ & $\begin{array}{c}\text { Follow-up } \\
\text { Periods (year) }\end{array}$ & $\begin{array}{l}\text { No. of Studies } \\
\text { Included }\end{array}$ & RRs & Significant? \\
\hline & \multirow{2}{*}{$\begin{array}{c}\text { Mayhew et al. } 2016 \\
\text { [5]/20 Prospective of } \\
20 \text { publications } \\
{[18-20,22-26,33,37-} \\
40,42-44,47-50]\end{array}$} & High- vs. Low & total nuts & $\begin{array}{l}243,795 \text { subjects } \\
\text { with } 13,726 \text { deaths }\end{array}$ & $40-80$ & $4-30$ & $\begin{array}{c}5 \text { studies from } 5 \\
\text { publications } \\
{[17-19,22,25]}\end{array}$ & $\begin{array}{c}0.73(95 \% \text { CI } 0.68 \\
0.78 ; p \text {-het }=0.31 \\
\left.I^{2}=16 \%\right)\end{array}$ & S \\
\hline & & Dose-response & total nuts & $\begin{array}{c}243,795 \text { subjects } \\
\text { with } 13,726 \text { deaths }\end{array}$ & $40-80$ & $4-30$ & $\begin{array}{c}5 \text { studies from } 5 \\
\text { publications } \\
{[17-19,22,25]}\end{array}$ & $\begin{array}{c}0.78(95 \% \text { CI } 0.63 \\
1.00)\end{array}$ & NS \\
\hline & \multirow{3}{*}{$\begin{array}{c}\text { Grosso et al. } 2015 \\
\text { [15]/9 prospective of } \\
9 \text { publications }[19,23- \\
25,38,39,50-52]\end{array}$} & High- vs. Low & total nuts & $\begin{array}{l}354,933 \text { subjects } \\
\text { with } 7775 \text { deaths }\end{array}$ & $40-80$ & $4-30$ & $\begin{array}{c}7 \text { studies from } 6 \\
\text { publications } \\
{[19,25,38,39,51,52]}\end{array}$ & $\begin{array}{c}0.71(95 \% \text { CI } 0.62 \\
0.81 ; p \text {-het }=0.24 \\
\left.I^{2}=25 \%\right)\end{array}$ & S \\
\hline & & \multirow{2}{*}{ Dose-response } & $\begin{array}{c}1 \text { serving of } \\
\text { total } \\
\text { nuts/week }\end{array}$ & $\begin{array}{l}354,933 \text { subjects } \\
\text { with } 7775 \text { deaths }\end{array}$ & $49-80$ & $4-30$ & $\begin{array}{c}4 \text { studies from } 4 \\
\text { publications } \\
{[19,25,38,51]}\end{array}$ & $\begin{array}{c}0.93 \text { (95\% CI 0.88 } \\
0.99 ; p \text {-het = 0.004; } \\
\left.\mathrm{I}^{2}=74 \%\right)\end{array}$ & $\mathrm{S}$ \\
\hline & & & $\begin{array}{l}1 \text { serving of } \\
\text { total nuts/day }\end{array}$ & $\begin{array}{l}354,933 \text { subjects } \\
\text { with } 7775 \text { deaths }\end{array}$ & $49-80$ & $4-30$ & $\begin{array}{c}4 \text { studies from } 4 \\
\text { publications } \\
{[19,25,38,51]}\end{array}$ & $\begin{array}{c}0.61(95 \% \text { CI } 0.42 \\
0.91 ; p \text {-het = 0.003; } \\
\left.\mathrm{I}^{2}=75 \%\right)\end{array}$ & S \\
\hline \multirow{6}{*}{$\begin{array}{c}\text { CHD } \\
\text { mortality }\end{array}$} & \multirow{4}{*}{$\begin{array}{l}\text { Chen et al. } 2017 \\
\text { [12]/18 prospective } \\
\text { studies from } 15 \\
\text { publications }[17-31]\end{array}$} & \multirow{3}{*}{ High- vs. Low } & total nuts & $\begin{array}{l}>429,833 \text { subjects } \\
\text { with } 10,083 \text { deaths }\end{array}$ & $40-80$ & $2-30$ & $\begin{array}{c}13 \text { studies from } 10 \\
\text { publications } \\
{[17-22,25-28]}\end{array}$ & $\begin{array}{c}0.73 \text { (95\% CI 0.67, } \\
0.80 ; p \text {-het = 0.30; } \\
\left.\mathrm{I}^{2}=14.2 \%\right)\end{array}$ & S \\
\hline & & & peanuts & $\begin{array}{l}265,252 \text { subjects } \\
\text { with } 7025 \text { deaths }\end{array}$ & $40-80$ & $2-30$ & $\begin{array}{c}5 \text { studies from } 3 \\
\text { publications [17-19] }\end{array}$ & $\begin{array}{c}0.76(95 \% \text { CI } 0.69 \\
0.82 ; p \text {-het = } 0.65 \\
\left.I^{2}=0 \%\right)\end{array}$ & S \\
\hline & & & tree nuts & $\begin{array}{c}130,987 \text { subjects } \\
\text { with } 6394 \text { deaths }\end{array}$ & $40-80$ & $2-30$ & $\begin{array}{c}3 \text { studies from } 2 \\
\text { publications }[17,19]\end{array}$ & $\begin{array}{c}0.79(95 \% \text { CI } 0.68 \\
0.92 ; p \text {-het = 0.25; } \\
\left.\mathrm{I}^{2}=27.5 \%\right)\end{array}$ & $\mathrm{S}$ \\
\hline & & Dose-response & $\begin{array}{l}\text { per } 1 \text { serving } \\
\text { of total nuts } \\
\text { per week }\end{array}$ & $\begin{array}{l}>412,892 \text { subjects } \\
\text { with } 10,399 \text { events }\end{array}$ & $40-80$ & $2-30$ & $\begin{array}{l}13 \text { studies from10 } \\
\text { publications } \\
{[17-22,25,26,28,31]}\end{array}$ & $\begin{array}{c}0.94(95 \% \text { CI 0.93, } \\
0.96 ; p \text {-het = 0.11; } \\
\left.\mathrm{I}^{2}=34.3 \%\right)\end{array}$ & S \\
\hline & \multirow{2}{*}{$\begin{array}{l}\text { Mayhew et al. } 2016 \\
\text { [5]/20 Prospective of } \\
20 \text { publications } \\
{[18-20,22-26,33,37-} \\
40,42-44,47-50]\end{array}$} & High- vs. Low & total nuts & $\begin{array}{l}278,584 \text { subjects } \\
\text { with } 8454 \text { events }\end{array}$ & $40-80$ & $2-30$ & $\begin{array}{c}7 \text { studies from } 7 \\
\text { publications } \\
{[17-19,22,24,26,39]}\end{array}$ & $\begin{array}{c}0.70 \text { (95\% CI } 0.64 \\
0.76 ; p \text {-het = } 0.65 \\
\left.\quad I^{2}=0 \%\right)\end{array}$ & S \\
\hline & & Dose-response & total nuts & $\begin{array}{l}278,584 \text { subjects } \\
\text { with } 8454 \text { events }\end{array}$ & $40-80$ & $2-30$ & $\begin{array}{c}7 \text { studies from } 7 \\
\text { publications } \\
{[17-19,22,24,26,39]}\end{array}$ & $\begin{array}{c}0.78(95 \% \text { CI } 0.57 \\
1.08)\end{array}$ & NS \\
\hline
\end{tabular}


Table 1. Cont.

\begin{tabular}{|c|c|c|c|c|c|c|c|c|c|}
\hline & Ref/Studies & Analyses & Nut Types & Sample Size & $\begin{array}{c}\text { SampleAge } \\
\text { (year) }\end{array}$ & $\begin{array}{c}\text { Follow-up } \\
\text { Periods (year) }\end{array}$ & $\begin{array}{l}\text { No. of Studies } \\
\text { Included }\end{array}$ & RRs & Significant? \\
\hline \multirow{6}{*}{$\begin{array}{c}\text { Stroke } \\
\text { mortality }\end{array}$} & \multirow{4}{*}{$\begin{array}{l}\text { Chen et al. } 2017 \\
\text { [12]/18 prospective } \\
\text { studies from } 15 \\
\text { publications }[17-31]\end{array}$} & \multirow{3}{*}{ High- vs. Low } & total nuts & $\begin{array}{l}449,293 \text { subjects } \\
\text { with } 4398 \text { deaths }\end{array}$ & $40-80$ & $2-30$ & $\begin{array}{c}12 \text { studies from } 9 \\
\text { publications [17-19, } \\
21,25,27,28,33,34]\end{array}$ & $\begin{array}{c}0.82(95 \% \text { CI } 0.73 \\
0.91 ; p \text {-het }=0.82 \\
\left.I^{2}=0 \%\right)\end{array}$ & S \\
\hline & & & peanuts & $\begin{array}{l}265,252 \text { subjects } \\
\text { with } 3315 \text { deaths }\end{array}$ & $40-80$ & $2-30$ & $\begin{array}{c}5 \text { studies from } 3 \\
\text { publications [17-19] }\end{array}$ & $\begin{array}{c}0.83(95 \% \text { CI } 0.71 \\
0.97 ; p \text {-het }=0.12 \\
\left.\mathrm{I}^{2}=46.2 \%\right)\end{array}$ & $\mathrm{S}$ \\
\hline & & & tree nuts & $\begin{array}{l}130,987 \text { subjects } \\
\text { with } 2130 \text { deaths }\end{array}$ & $40-80$ & $2-30$ & $\begin{array}{c}3 \text { studies from } 2 \\
\text { publications }[17,19]\end{array}$ & $\begin{array}{c}0.93 \text { (95\% CI 0.77, } \\
1.13 ; p \text {-het }=0.44 \\
\left.I^{2}=0 \%\right)\end{array}$ & NS \\
\hline & & Dose-response & $\begin{array}{l}\text { per } 1 \text { serving } \\
\text { of total nuts } \\
\text { per week }\end{array}$ & $\begin{array}{l}>432,352 \text { subjects } \\
\text { with } 4831 \text { deaths }\end{array}$ & $40-80$ & $2-30$ & $\begin{array}{c}12 \text { studies from } 9 \\
\text { publications [17-19, } \\
21,25,28,31,33,34]\end{array}$ & $\begin{array}{c}0.95 \text { (95\% CI 0.91, } \\
0.997 ; p \text {-het = 0.005; } \\
\left.\mathrm{I}^{2}=60.6 \%\right)\end{array}$ & NS \\
\hline & $\begin{array}{l}\text { Mayhew et al. } 2016 \\
\text { [5]/20 Prospective }\end{array}$ & High- vs. Low & total nuts & $\begin{array}{l}159,322 \text { subjects } \\
\text { with } 2166 \text { events }\end{array}$ & $29.2-69$ & $8.3-30$ & $\begin{array}{c}3 \text { studies from } 3 \\
\text { publications } \\
{[17,19,33]}\end{array}$ & $\begin{array}{c}0.83(95 \% \text { CI } 0.69 \\
1.00 ; p \text {-het = 0.54; } \\
\left.I^{2}=0 \%\right)\end{array}$ & NS \\
\hline & $\begin{array}{c}\text { of } 20 \text { publications } \\
\text { [18-20,22-26,33,37- } \\
40,42-44,47-50]\end{array}$ & Dose-response & total nuts & $\begin{array}{l}159,322 \text { subjects } \\
\text { with } 2166 \text { events }\end{array}$ & $29.2-69$ & $8.3-30$ & $\begin{array}{c}3 \text { studies from } 3 \\
\text { publications } \\
{[17,19,33]}\end{array}$ & $\begin{array}{c}0.85 \text { (95\% CI } 0.55 \\
1.31)\end{array}$ & NS \\
\hline \multirow{5}{*}{$\begin{array}{l}\text { Diabetes } \\
\text { Mortality }\end{array}$} & \multirow{5}{*}{$\begin{array}{c}\text { Aune et al. } 2016 \\
\text { [14]/20 prospective } \\
\text { studies from } 9 \\
\text { publications } \\
{[17-29,31-46]}\end{array}$} & \multirow[t]{2}{*}{ High- vs. Low } & peanuts & $\begin{array}{l}265,252 \text { subjects } \\
\text { with } 901 \text { deaths }\end{array}$ & $40-79$ & $4-30$ & $\begin{array}{c}5 \text { studies from } 3 \\
\text { publications [17-19] }\end{array}$ & $\begin{array}{c}0.84(95 \% \text { CI 0.60, } \\
1.19 ; p \text {-het = 0.14; } \\
\left.\mathrm{I}^{2}=42.6 \%\right)\end{array}$ & NS \\
\hline & & & tree nuts & $\begin{array}{l}130,987 \text { subjects } \\
\text { with } 4622 \text { deaths }\end{array}$ & $40-79$ & $4-30$ & $\begin{array}{c}3 \text { studies from } 2 \\
\text { publications }[18,19]\end{array}$ & $\begin{array}{c}1.19(95 \% \text { CI } 0.74 \\
1.89 ; p \text {-het }=0.43 ; \\
\left.\quad I^{2}=0 \%\right)\end{array}$ & NS \\
\hline & & \multirow{3}{*}{ Dose-response } & total nuts & $\begin{array}{l}202,751 \text { subjects } \\
\text { with } 800 \text { deaths. }\end{array}$ & $40-79$ & $4-30$ & $\begin{array}{c}4 \text { studies from } 3 \\
\text { publications [17-19] }\end{array}$ & $\begin{array}{c}0.61(95 \% \text { CI } 0.43 \\
0.88 ; p \text {-het }=0.76 \\
\left.I^{2}=0 \%\right)\end{array}$ & $\mathrm{S}$ \\
\hline & & & peanuts & $\begin{array}{l}265,252 \text { subjects } \\
\text { with } 901 \text { deaths }\end{array}$ & $40-79$ & $4-30$ & $\begin{array}{c}5 \text { studies from } 3 \\
\text { publications [17-19] }\end{array}$ & $\begin{array}{c}0.73(95 \% \text { CI } \\
0.45-1.20 ; p \text {-het }= \\
\left.0.32 ; \mathrm{I}^{2}=15.4 \%\right)\end{array}$ & NS \\
\hline & & & tree nuts & $\begin{array}{l}130,987 \text { subjects } \\
\text { with } 4622 \text { deaths }\end{array}$ & $55-61$ & $10-30$ & $\begin{array}{c}3 \text { studies from } 2 \\
\text { publications }[17,19]\end{array}$ & $\begin{array}{c}1.23(95 \% \text { CI } 0.68 \\
2.25 ; p \text {-het }=0.62 ; \\
\left.\quad I^{2}=0 \%\right)\end{array}$ & NS \\
\hline
\end{tabular}

CHD, coronary heart disease; CI, confidence interval; CVD, cardiovascular disease; het, heterogeneity; RCT, randomized-controlled trial; Ref, references; RR, relative risk. 


\subsubsection{Incidence of Type 2 Diabetes, CVD, Hypertension, CHD and Stroke}

A summary of nut consumption and incidence of T2DM, CVD, hypertension, CHD and stroke is shown in Table 2. Most meta-analyses were analyzed with prospective studies while two meta-analyses by Afshine et al. 2014 [57] and Weng et al. 2016 [58] were analyzed with prospective and intervention studies in Table 2. Forest plots for Table 2 are presented in Supplementary Figures S11-S21.

\section{Type 2 Diabetes}

Most meta-analyses have found no relationship with diabetes incidence but Wu et al. 2015 [59] has shown a $2 \%$ lower incidence. On the other hand, a dose response analysis meta-analysis [57] showed a $13 \%$ reduction in diabetes incidence per 4 servings/week.

Schwingshackl et al. 2017 [60] examined the association between food groups and incidence of T2DM in prospective studies with 27,016 occurrences of T2DM comparing the highest with the lowest nut consumption (average nut consumption from lowest to highest ntile (quartile or quintile) ranging from 0 to $27 \mathrm{~g} /$ day). They found no significant association between nut consumption and risk of T2DM with a RR of $0.95\left(95 \%\right.$ CI $0.85,1.05 ; p$-het $\left.=0.004 ; \mathrm{I}^{2}=67 \%\right)$ in 8 prospective studies (6 publications) $[53-56,61,62]$ while a $28 \mathrm{~g} /$ day increase did not significantly attenuate the risk of T2DM $\left(\mathrm{RR}=0.89 ; 95 \% \mathrm{CI} 0.71,1.12 ; p\right.$-het $\left.=0.0002 ; \mathrm{I}^{2}=77 \%\right)$ in 7 prospective studies ( 5 publications) $[53-56,61]$. However, given the marked heterogeneity this finding is not reliable. This finding was consistent with other meta-analyses $[16,63,64]$. If body mass index (BMI) is not adjusted for a relationship with T2DM can be seen. Luo et al. 2014 [16] showed a one serving/day increase in total nut consumption resulted in a RR of $0.88\left(95 \%\right.$ CI $0.84,0.92 ; p$-het $\left.=0.60 ; \mathrm{I}^{2}=0.0 \%\right)$ after adjustment for age only (4 studies [53-56]). In a meta-analysis of 6 studies (5 publications) $[53,55,56,65,66]$ (5 prospective cohorts and 1 randomized controlled trial (RCT; 230,216 subjects with 13,308 events) conducted by Afshin et al. 2014 [57], nut consumption per 4 servings/week decreased the risk of T2DM by 13\% $\left(\mathrm{RR}=0.87 ; 95 \% \mathrm{CI} 0.81,0.94 ; p\right.$-het $\left.=0.269 ; \mathrm{I}^{2}=21.8 \%\right)$ [57]. Given that nuts are associated with a lower BMI adjustment for BMI may obscure the true relationship between nuts and T2DM which is directly mediated via lower BMI.

\section{Cardiovascular Disease}

Three meta-analyses $[5,14,16]$ of prospective studies that examined high and low consumption of nuts have shown a lower incidence of CVD. Aune et al. 2016 [14] showed a 19\% reduction while Mayhew et al. 2016 [5] and Luo et al. 2014 [16] showed a 44\% and a 30\% reduction, respectively. However, Mayhew et al. 2016 [5] and Luo et al. 2014 [16] just analyzed only one and four studies, respectively.

Aune et al. 2016 [14] found nut consumption was inversely associated with total CVD incidence with a RR of $0.81\left(95 \%\right.$ CI $0.74,0.89 ; p$-het $\left.=0.02 ; \mathrm{I}^{2}=52.3 \%\right)$ from 12 cohort studies (12 publications) $[17-19,21,22,25,27-29,31,35,36]$ comparing the highest with the lowest intakes. Consumption of tree nuts or peanuts showed a similar effect on CVD incidence with RRs of 0.81 . The RR per $28 \mathrm{~g} /$ day increase in nuts from 12 cohort studies (12 publications) [17-19,21,22,25,27-29,31,35,36] was 0.79 ( $95 \%$ CI $0.70,0.88)$. RRs were similar per $28 \mathrm{~g} /$ day increase in peanuts $(R R=0.64)$ or tree nuts $(R R=0.75)[14]$.

\section{Hypertension}

Meta-analyses of prospective studies $[63,64,67]$ have found a $\sim 15 \%$ lower risk of hypertension comparing the highest to the lowest nut intakes.

Schwingshackl et al. 2017 [67] examined the association between 12 food groups (whole grains, refined grains, vegetables, fruits, legumes, nuts, dairy, fish, eggs, red meat, processed meat and sugar-sweetened beverages) and incidence of hypertension in a meta-analysis of prospective studies. This meta-analysis of 4 studies [68-71] showed that $28 \mathrm{~g} /$ day of nuts attenuated the risk of hypertension 
by $30 \%$ (RR: $0.70 ; 95 \%$ CI $0.45,1.08 ; \mathrm{I}^{2}=69 \%$; $p$-het $\left.=0.02\right)$. The RR was $0.85\left(95 \%\right.$ CI $0.78,0.92 ; \mathrm{I}^{2}=0 \%$; $p$-het $=0.92)$ in a comparison of high versus low nut consumption.

Guo et al. 2015 [63] found that nut consumption, especially at $>2$ servings/week was associated with a lower risk of hypertension. Comparing the highest with the lowest categories of nut consumption, a $16 \%$ decrease in the incidence of hypertension was observed (RR $=0.84 ; 95 \% \mathrm{CI}$ : 0.76 , $0.93)$ in 3 studies from 3 publications $[68,70,72]$.

Zhou et al. 2014 [64] also found the highest nut consumption decreased the risk of hypertension by $15 \%\left(\mathrm{RR}=0.85 ; 95 \% \mathrm{CI} 0.79,0.92 ; p\right.$-het $\left.=0.927 ; \mathrm{I}^{2}=0.0 \%\right)$ compared with the lowest nut consumption in an analysis of 4 studies from 4 publications [68,70-72] involving 40,102 subjects with 12,814 events.

\section{Coronary Heart Disease}

The incidence of CHD (or CAD) was lowered by $17-34 \%$ as shown in 7 meta- analyses $[5,14,16,58$, $64,73,74]$ of prospective studies.

Aune et al. 2016 [14] found that nut consumption decreased the risk of CHD by $24 \%$ $(\mathrm{RR}=0.76 ; 95 \% \mathrm{CI} 0.69,0.84)$ comparing the highest with the lowest intakes. This finding came from 11 studies from 10 publications [17-19,22,26-28,37-39] involving 315,397 subjects with 12,331 events. Consumption of either tree nuts or peanuts also showed similar reductions in CHD risk with RRs of 0.79 and 0.76 respectively. An increase in 1 serving/day resulted in a 21\% decrease in CHD $(\mathrm{RR}=0.79 ; 95 \% \mathrm{CI} 0.70,0.88)$ in 12 studies from 12 publications $[17-19,21,22,25,27-29,31,35,36](376,228$ subjects with 18,655 events). Similar results were seen in earlier meta-analyses [5,16,58,64,74].

Bechthold et al. 2017 [73] showed a significant inverse association between nut consumption and risk of $\mathrm{CHD}\left(\mathrm{RR}=0.80 ; 95 \% \mathrm{CI} 0.62,1.03 ; \mathrm{I}^{2}=79 \%, p\right.$-het $\left.=0.002\right)$ in 4 prospective cohort studies from 4 publications [37-40] with 5480 cases of CHD comparing groups with the highest and the lowest nut consumption (overall nut consumption ranging from 0 to $28 \mathrm{~g} /$ day). An additional intake of $28 \mathrm{~g} /$ day of nuts showed a 33\% reduction in the risk of CHD ( $R R=0.67 ; 95 \%$ CI $0.43,1.05, \mathrm{I}^{2}=85 \%$, $p$-het $\left.=0.001\right)$. A non-linear dose-response was seen. An increase in nut consumption up to 10-15 g/day reduced the incidence of $\mathrm{CHD}$ by $\sim 21 \%$.

\section{Fatal/Non-Fatal Coronary Heart Disease}

A meta-analysis by Afshin et al. 2014 [57] showed that 4 serves/week of nuts lowered the risk of fatal CHD (RR = 0.76, 95\% CI 0.69, 0.84; $p$-het $\left.=0.227 ; \mathrm{I}^{2}=27.7 \%\right)$ and nonfatal CHD $(\mathrm{RR}=0.78,95 \% \mathrm{CI}$ $0.67,0.92 ; p$-het $=0.463 ; \mathrm{I}^{2}=0.0 \%$ ) by $24 \%$ and $22 \%$, respectively. Per daily serve they found significant reductions in risk of non-fatal CHD and fatal CHD with RRs of 0.78 and 0.76 respectively. Non-fatal CHD was analyzed from 4 studies (4 publications) [2,38,39,47] involving 141,390 subjects with 2101 events, while fatal CHD events came from 6 studies (5 publications) $[2,19,22,38,39]$ involving 206,114 subjects with 6749 events.

Stroke

All meta-analyses apart from two [14,75] which showed a 10-11\% reduction in stroke incidence, have found no effect of nuts on stroke incidence. However, the meta-analysis of Aune et al. 2016 [14] is much more convincing as it included a greater number of studies compared with other meta-analyses $[16,57,64,73]$.

Aune et al. 2016 [14] reported a 11\% reduction ( $R R=0.89 ; 95 \%$ CI $0.82,0.97 ; p$-het $\left.=0.90 ; I^{2}=0 \%\right)$ in the risk of stroke comparing the highest with the lowest nut consumption. This finding came from 10 studies (9 publications) [17,18,27,28,33,34,41-43] involving 396,768 subjects with 9272 events. One serve/day of reduced the risk of stroke by $7 \%\left(R R=0.93 ; 95 \%\right.$ CI $0.83,1.05 ; p$-het $\left.=0.31 ; I^{2}=13.7 \%\right)$ in 11 studies (9 publications) [17,18,27,28,33,34,41-43].

Shi et al. 2014 [75] also found an inverse association between nut consumption and risk of stroke in 4 prospective studies (3 publications) [34,42,43] including 228,799 subjects with 5669 strokes. Nut consumption reduced total stroke risk by $10 \%\left(R R=0.90 ; 95 \%\right.$ CI $0.81,0.99 ; p$-het $\left.=0.527, \mathrm{I}^{2}=0\right)$ 
comparing the highest with the lowest nut consumption. Female nut consumers ( $\mathrm{RR}=0.85,95 \% \mathrm{CI}$ $0.75,0.97$ ) showed a lower incidence of stroke than male nut consumers ( $R R=0.95,95 \% \mathrm{CI} 0.82,1.11)$.

However, Bechthold et al. 2017 [73] found no association between nut consumption and the risk of stroke $\left(\mathrm{RR}=0.94 ; 95 \% \mathrm{CI} 0.85,1.05, \mathrm{I}^{2}=18 \%\right.$, $p$-het $\left.=0.30\right)$ in 6 studies (6 publications) $[33,41-44,76]$ with 7490 cases for stroke which compared the highest with the lowest nut consumption (ranging from 0-30 g/day). An increase of $28 \mathrm{~g} /$ day was not associated with stroke ( $\mathrm{RR}=0.99 ; 95 \% \mathrm{CI}$ $0.84,1.17, \mathrm{I}^{2}=45 \%$, $p$-het $=0.11$ ) in a non-linear dose-response. In addition, other meta-analyses found no association between nut consumption and risk of stroke $[16,57,64]$. In comparison with the meta-analysis by Aune et al. 2016 [14], these meta-analyses [16,57,64,73] included fewer studies.

\subsubsection{Body Weight}

Li et al. 2018 [77] conducted a meta-analysis of prospective studies to examine the association between nut consumption and the risk of metabolic syndrome or overweight/obesity or obesity alone. The RR per one serving/week of nut consumption increase from 3 studies [78-80] was 0.96 (95\% CI $\left.0.92,0.99 ; \mathrm{I}^{2}=0\right)$ for risk of metabolic syndrome. The RRs for overweight/obesity $(0.97 ; 95 \%$ CI 0.95 , $\left.0.98 ; \mathrm{I}^{2}=0\right)$ and obesity $\left(0.95 ; 95 \% \mathrm{CI} 0.89,1.02 ; \mathrm{I}^{2}=74 \%\right)$ were similar per one serving/week of nuts from 2 studies [81,82] and 2 studies [82,83], respectively.

Pooled results of the slope coefficient from a meta-analysis of epidemiological studies, which was performed by Grosso et al. 2015 [15], showed that nut consumption was inversely associated with BMI (0.15\% (slope coefficient: -0.15 ; SE: $-0.24,-0.06))$ and smoking $(-0.59 \%$ (slope coefficient: $0.59 ; \mathrm{SE}-0.99,-0.20)$ ). An additional one serving of nuts per week was associated with increased consumption of vegetables ( 13 g/day (slope coefficient: 13.28; SE: 6.09, 20.46)), fruits ( 10 g/day (slope coefficient: 9.82; SE: 4.5, 15.14)) and alcohol ( 1 g/day (slope coefficient: 0.99; SE: 0.79, 1.20)) [15]. 
Table 2. Summary of nut consumption and incidence of T2DM, CVD, hypertension, CHD and stroke.

\begin{tabular}{|c|c|c|c|c|c|c|c|c|c|}
\hline Variables & Ref/Studies & Analyses & Nut Types & Sample Size & $\begin{array}{l}\text { Sample } \\
\text { Age }\end{array}$ & $\begin{array}{l}\text { Follow-up } \\
\text { Periods }\end{array}$ & $\begin{array}{l}\text { No. of Studies } \\
\text { Included }\end{array}$ & RRs & Effects \\
\hline \multirow{10}{*}{ T2DM } & \multirow{2}{*}{$\begin{array}{l}\text { Schwingshackl et al. } \\
2017 \text { [60]/8 prospective } \\
\text { studies from } 7 \\
\text { publications } \\
{[36,53-56,61,62]}\end{array}$} & High vs. Low & total nuts & 27,016 events & $20-80$ & $4-19.2$ & $\begin{array}{c}8 \text { studies from } 7 \\
\text { publications } \\
{[36,53-56,61,62]}\end{array}$ & $\begin{array}{l}0.95(95 \% \text { CI 0.85, 1.05; } \\
\left.p \text {-het }=0.004 ; \mathrm{I}^{2}=67 \%\right)\end{array}$ & NS \\
\hline & & $\begin{array}{l}\text { Dose response: } \\
28 \mathrm{~g} / \text { day } \\
\text { increase }\end{array}$ & total nuts & 27,016 events & $20-80$ & $4-19.2$ & $\begin{array}{l}7 \text { studies from } 6 \\
\text { publications } \\
{[36,53-56,61]}\end{array}$ & $\begin{array}{c}0.89(95 \% \text { CI } 0.71,1.12 ; \\
\left.p \text {-het }=0.0002 ; I^{2}=77 \%\right)\end{array}$ & NS \\
\hline & $\begin{array}{c}\text { Wu et al. } 2015[59] /(5 \\
\text { prospective studies } \\
\text { from } 5 \text { publications } \\
[53-56,84])\end{array}$ & High vs. Low & total nuts & $\begin{array}{l}\text { 263,406 subjects } \\
\text { with } 11,610 \\
\text { events }\end{array}$ & $20-87$ & $4-19.2$ & $\begin{array}{l}5 \text { studies from } 5 \\
\text { publications } \\
{[53-56,84]}\end{array}$ & $\begin{array}{c}0.98(95 \% \text { CI } 0.84,1.14 ; \\
\left.p \text {-het }=0.004 ; I^{2}=74.2 \%\right)\end{array}$ & NS \\
\hline & \multirow{4}{*}{$\begin{array}{c}\text { Guo et al. } 2015[63] / 8 \\
\text { prospective studies } \\
\text { from } 8 \text { publications } \\
{[53-56,65,68,70,72]}\end{array}$} & High vs. Low & total nuts & $\begin{array}{l}263,663 \text { subjects } \\
\text { with } 11,580 \\
\text { events }\end{array}$ & $35-77$ & $4.6-23$ & $\begin{array}{c}5 \text { studies from } 5 \\
\text { publications } \\
{[53-56,65]}\end{array}$ & $\begin{array}{c}0.98(95 \% \text { CI } 0.84,1.15 \\
\left.p \text {-het }=0.008, I^{2}=67.7 \%\right)\end{array}$ & NS \\
\hline & & \multirow{3}{*}{ Dose-response: } & $\begin{array}{c}<1 \text { serving nut } \\
\text { per week }\end{array}$ & $\begin{array}{l}263,663 \text { subjects } \\
\text { with } 11,580 \\
\text { events }\end{array}$ & $35-77$ & $4.6-23$ & $\begin{array}{c}3 \text { studies from } 3 \\
\text { publications } \\
\text { [53-55] }\end{array}$ & $\begin{array}{r}1.00(95 \% \text { CI 0.95, 1.04; } \\
\left.p \text {-het = 0.789, } \mathrm{I}^{2}=0.0 \%\right)\end{array}$ & NS \\
\hline & & & $\begin{array}{l}1 \text { to } 4 \text { servings } \\
\text { nut per week }\end{array}$ & $\begin{array}{l}263,663 \text { subjects } \\
\text { with } 11,580 \\
\text { events }\end{array}$ & $35-77$ & $4.6-23$ & $\begin{array}{l}3 \text { studies from } 3 \\
\text { publications } \\
\text { [53-55] }\end{array}$ & $\begin{array}{r}1.03(95 \% \text { CI 0.98, 1.08; } \\
\left.p \text {-het }=0.903, I^{2}=0.0 \%\right)\end{array}$ & NS \\
\hline & & & $\begin{array}{l}\geq 5 \text { servings } \\
\text { nut per week }\end{array}$ & $\begin{array}{l}263,663 \text { subjects } \\
\text { with } 11,580 \\
\text { events }\end{array}$ & $35-77$ & $4.6-23$ & $\begin{array}{c}3 \text { studies from } 3 \\
\text { publications } \\
\text { [53-55] }\end{array}$ & $\begin{array}{c}1.04(95 \% \text { CI } 0.95,1.14 ; \\
\left.p \text {-het }=0.067, \mathrm{I}^{2}=58.1 \%\right)\end{array}$ & NS \\
\hline & $\begin{array}{c}\text { Afshin et al. } 2014 \\
\text { [57]/16 studies } \\
\text { (14 prospective } \\
{[2,19,22,38-40,42,43,47} \\
53,55,56,65,85,86] \& \\
2 \text { RCTs }[2,66]\end{array}$ & $\begin{array}{l}\text { Dose-response: } \\
\text { for } 4 \text { servings } \\
(28.4 \mathrm{~g}) / \text { week of } \\
\text { total nuts }\end{array}$ & total nuts & $\begin{array}{l}230,216 \text { subjects } \\
\text { with } 13,308 \\
\text { events }\end{array}$ & $35-77$ & $4-19.2$ & $\begin{array}{l}6 \text { studies from } 5 \\
\text { publications } \\
{[53,55,56,65,66]}\end{array}$ & $\begin{array}{c}0.87(95 \% \text { CI } 0.81,0.94 \\
\left.p \text {-het }=0.269 ; \mathrm{I}^{2}=21.8 \%\right)\end{array}$ & S \\
\hline & \multirow{2}{*}{$\begin{array}{c}\text { Zhou et al. } 2014 \\
\text { [64]/23 prospective } \\
\text { from } 19 \text { publications } \\
{[22,34,38,40,42,43,48} \\
53-56,68,70-72,86-89]\end{array}$} & High vs. Low & total nuts & $\begin{array}{l}342,213 \text { subjects } \\
\text { with } 14,400 \\
\text { events }\end{array}$ & $34-87$ & $4.6-19.2$ & $\begin{array}{c}6 \text { studies from } 5 \\
\text { publications } \\
{[53-56,86]}\end{array}$ & $\begin{array}{c}0.92(95 \% \text { CI } 0.78,1.09 \\
\left.p \text {-het }=0.001 ; I^{2}=78.7 \%\right)\end{array}$ & NS \\
\hline & & $\begin{array}{l}\text { Dose-response: } \\
1 \text { serving/day } \\
\text { increase }\end{array}$ & total nuts & $\begin{array}{c}342,213 \text { subjects } \\
\text { with } 14,400 \\
\text { events }\end{array}$ & $34-87$ & $4.6-19.2$ & $\begin{array}{c}6 \text { studies from } 5 \\
\text { publications } \\
{[53-56,86]}\end{array}$ & $\begin{array}{c}0.80(95 \% \text { CI } 0.57,1.14 ; \\
\left.p \text {-het }<0.001 ; I^{2}=87.1 \%\right)\end{array}$ & NS \\
\hline
\end{tabular}


Table 2. Cont

\begin{tabular}{|c|c|c|c|c|c|c|c|c|c|}
\hline Variables & Ref/Studies & Analyses & Nut Types & Sample Size & $\begin{array}{l}\text { Sample } \\
\text { Age }\end{array}$ & $\begin{array}{c}\text { Follow-up } \\
\text { Periods }\end{array}$ & $\begin{array}{l}\text { No. of Studies } \\
\text { Included }\end{array}$ & RRs & Effects \\
\hline & \multirow{5}{*}{$\begin{array}{c}\text { Luo et al. } 2014 \text { [16]/18 } \\
\text { prospective studies } \\
\text { from } 18 \text { publications } \\
{[19,20,22,24-26,38-40,} \\
42-44,48,50,53-56]\end{array}$} & \multirow{2}{*}{$\begin{array}{c}\text { High }(\geq 2 \text { to } \geq 7 \\
\text { servings / week) } \\
\text { vs. Low ( } 0 \text { to } \leq 1 \\
\text { serving/week) }\end{array}$} & total nuts & $\begin{array}{l}2,982,852 \\
\text { subjects with } \\
12,655 \text { events }\end{array}$ & $35-72$ & 4 & $\begin{array}{c}1 \text { study from } 1 \\
\text { publication [55] }\end{array}$ & $\begin{array}{c}0.83(95 \% \text { CI } 0.74,0.93 \\
\left.p \text {-het }=0.278 ; I^{2}=14.9 \%\right) \\
\text { in multivariable-adjusted } \\
\text { model without BMI }\end{array}$ & $\mathrm{S}$ \\
\hline & & & total nuts & $\begin{array}{l}2,982,852 \\
\text { subjects with } \\
14,486 \text { events }\end{array}$ & $35-77$ & $4-19.2$ & $\begin{array}{l}4 \text { studies from } 4 \\
\text { publications } \\
\text { [53-56] }\end{array}$ & $\begin{array}{c}1.00(95 \% \text { CI } 0.84,1.19 ; \\
\left.p \text {-het }=0.008 ; I^{2}=67.7 \%\right) \\
\text { in multivariable-adjusted } \\
\text { model with BMI }\end{array}$ & NS \\
\hline & & \multirow{3}{*}{$\begin{array}{l}\text { Dose-response: } \\
1 \text { serving ( } 28 \\
\text { g)/day increase } \\
\text { in total nuts }\end{array}$} & total nuts & $\begin{array}{c}2,982,852 \\
\text { subjects with } \\
14,486 \text { events }\end{array}$ & $35-77$ & $4-19.2$ & $\begin{array}{c}4 \text { studies from } 4 \\
\text { publications } \\
\text { [53-56] }\end{array}$ & $\begin{array}{c}0.88(95 \% \text { CI } 0.84,0.92 ; \\
\left.p \text {-het }=0.60 ; I^{2}=0.0 \%\right) \\
\text { after adjustment for age }\end{array}$ & S \\
\hline & & & total nuts & $\begin{array}{c}116,4248 \\
\text { subjects with } \\
5121 \text { events }\end{array}$ & $35-77$ & 4 & $\begin{array}{c}1 \text { study from } 1 \\
\text { publication [55] }\end{array}$ & $\begin{array}{c}0.80(95 \% \text { CI } 0.69-0.94 ; \\
\left.p \text {-het }=0.151 ; \mathrm{I}^{2}=51.4 \%\right) \\
\text { in multivariable-adjusted } \\
\text { model without BMI }\end{array}$ & S \\
\hline & & & total nuts & $\begin{array}{l}2,918,625 \\
\text { subjects with } \\
13,878 \text { events }\end{array}$ & $35-77$ & $4-19.2$ & $\begin{array}{c}3 \text { studies from } 3 \\
\text { publications } \\
\text { [53-55] }\end{array}$ & $\begin{array}{c}1.03(95 \% \text { CI } 0.91,1.16 ; \\
\left.p \text {-het }=0.04 ; I^{2}=63.9 \%\right) \\
\text { in multivariable-adjusted } \\
\text { model with BMI }\end{array}$ & NS \\
\hline \multirow{3}{*}{ Total CVD } & \multirow{3}{*}{$\begin{array}{c}\text { Aune et al. } 2016[14] / 20 \\
\text { prospective studies } \\
\text { from } 29 \text { publications } \\
{[17-29,31-46]}\end{array}$} & \multirow{3}{*}{ High vs. Low } & total nuts & $\begin{array}{l}376,228 \text { subjects } \\
\text { with } 18,655 \\
\text { events }\end{array}$ & $35-79$ & $4-30$ & $\begin{array}{c}12 \text { studies from } 12 \\
\text { publications } \\
{[17-19,21,22,25} \\
27-29,31,35,36] \\
\end{array}$ & $\begin{array}{r}0.81(95 \% \text { CI } 0.74,0.89 \\
\left.p \text {-het }=0.02 ; \mathrm{I}^{2}=52.3 \%\right)\end{array}$ & S \\
\hline & & & peanuts & $\begin{array}{l}265,252 \text { subjects } \\
\text { with } 12,043 \\
\text { events }\end{array}$ & $40-79$ & $4-30$ & $\begin{array}{c}5 \text { studies from } 3 \\
\text { publications } \\
\text { [17-19] }\end{array}$ & $\begin{array}{c}0.76(95 \% \text { CI } 0.70,0.81 \\
\left.p \text {-het }=0.90 ; \mathrm{I}^{2}=0 \%\right)\end{array}$ & S \\
\hline & & & tree nuts & $\begin{array}{l}\text { 130,987 subjects } \\
\text { with } 9456 \\
\text { events }\end{array}$ & $55-61$ & $10-30$ & $\begin{array}{c}3 \text { studies from } 2 \\
\text { publications } \\
{[17,19]}\end{array}$ & $\begin{array}{c}0.76(95 \% \text { CI } 0.69-0.84 \\
\left.p \text {-het }=0.92 ; \mathrm{I}^{2}=0 \%\right)\end{array}$ & S \\
\hline
\end{tabular}


Table 2. Cont.

\begin{tabular}{|c|c|c|c|c|c|c|c|c|c|}
\hline Variables & Ref/Studies & Analyses & Nut Types & Sample Size & $\begin{array}{c}\text { Sample } \\
\text { Age }\end{array}$ & $\begin{array}{l}\text { Follow-up } \\
\text { Periods }\end{array}$ & $\begin{array}{l}\text { No. of Studies } \\
\text { Included }\end{array}$ & RRs & Effects \\
\hline & & \multirow{3}{*}{$\begin{array}{c}\text { Dose-response: } \\
28 \mathrm{~g} / \text { day } \\
\text { increase in total } \\
\text { nuts, } 10 \mathrm{~g} / \text { day } \\
\text { increase in } \\
\text { peanuts and } \\
\text { tree nuts }\end{array}$} & total nuts & $\begin{array}{c}376,228 \text { subjects } \\
\text { with } 18,655 \text { events }\end{array}$ & $35-89$ & $4-30$ & $\begin{array}{c}12 \text { studies from } 12 \\
\text { publications } \\
{[17-19,21,22,25,27-29} \\
31,35,36]\end{array}$ & $\begin{array}{c}0.79 \text { (95\% CI 0.70, } 0.88 \\
p \text {-het }=0.004 \\
\left.\mathrm{I}^{2}=59.6 \%\right)\end{array}$ & $S$ \\
\hline & & & peanuts & $\begin{array}{c}265,252 \text { subjects } \\
\text { with } 12,043 \text { events }\end{array}$ & $40-79$ & $4-30$ & $\begin{array}{c}5 \text { studies from } 2 \\
\text { publications [17-19] }\end{array}$ & $\begin{array}{c}0.64(95 \% \text { CI } 0.50-0.81 \\
\left.p \text {-het }=0.001 ; \mathrm{I}^{2}=77 \%\right)\end{array}$ & S \\
\hline & & & tree nuts & $\begin{array}{l}130,987 \text { subjects } \\
\text { with } 9456 \text { events }\end{array}$ & $40-79$ & $4-30$ & $\begin{array}{c}3 \text { studies from } 2 \\
\text { publications }[17,19]\end{array}$ & $\begin{array}{c}0.75(95 \% \text { CI } 0.67-0.84 ; \\
\left.p \text {-het }=0.84 ; I^{2}=0 \%\right)\end{array}$ & $S$ \\
\hline & \multirow{2}{*}{$\begin{array}{c}\text { Mayhew et al. } 2016 \\
\text { [5]/20 prospective } \\
\text { studies from } 20 \\
\text { publications } \\
{[18-20,22-26,33,37-40} \\
42-44,47-50]\end{array}$} & High vs. Low & total nuts & $\begin{array}{c}6309 \text { women with } \\
\text { diabetes } 634 \\
\text { events }\end{array}$ & $44.3-67.6$ & 22 & $\begin{array}{c}1 \text { study from } 1 \\
\text { publication [48] }\end{array}$ & $\begin{array}{c}0.56(95 \% \text { CI } 0.36-0.88 \\
\left.p \text {-het }=0.54 ; I^{2}=0 \%\right)\end{array}$ & S \\
\hline & & $\begin{array}{c}\text { Dose-response: } \\
4 \\
\text { servings/week } \\
\text { increase }\end{array}$ & total nuts & $\begin{array}{l}6309 \text { women with } \\
\text { diabetes } 634 \\
\text { events }\end{array}$ & $44.3-67.6$ & 22 & $\begin{array}{c}1 \text { study from } 1 \\
\text { publication [48] }\end{array}$ & $0.72(95 \%$ CI $0.55-0.96)$ & $S$ \\
\hline & \multirow{2}{*}{$\begin{array}{l}\text { Luo et al. } 2014[16] / 18 \\
\text { prospective studies } \\
\text { from } 18 \text { publications } \\
{[19,20,22,24-26,38-40} \\
42-44,48,50,53-56]\end{array}$} & $\begin{array}{l}\text { High }(\geq 2 \text { to } \geq 7 \\
\text { servings / week) } \\
\text { vs. Low ( } 0 \text { to } \leq 1 \\
\text { serving/week) }\end{array}$ & total nuts & 8862 events & $52-80$ & $4.8-30$ & $\begin{array}{c}4 \text { studies from } 4 \\
\text { publications } \\
{[19,22,25,48]}\end{array}$ & $\begin{array}{c}0.70(95 \% \text { CI } 0.60,0.81 \\
p \text {-het }=0.274 \\
\left.\mathrm{I}^{2}=22.8 \%\right)\end{array}$ & $\mathrm{S}$ \\
\hline & & $\begin{array}{l}\text { Dose-response: } \\
1 \text { serving ( } 28 \\
\text { g)/day increase }\end{array}$ & total nuts & 8862 events & $52-80$ & $4.8-30$ & $\begin{array}{c}4 \text { studies from } 4 \\
\text { publications } \\
[19,22,25,48])\end{array}$ & $\begin{array}{c}0.71(95 \% \text { CI } 0.59,0.85 \\
p \text {-het }=0.119 ; \\
\left.\mathrm{I}^{2}=48.8 \%\right)\end{array}$ & $\mathrm{S}$ \\
\hline \multirow{5}{*}{ Hypertension } & \multirow{2}{*}{$\begin{array}{l}\text { Schwingshackl et al. } \\
2017 \text { [67]/4 prospective } \\
\text { studies from } 4 \\
\text { publications [68-71] }\end{array}$} & High vs. Low & total nuts & 11,962 events & $20-95$ & $3-9$ & $\begin{array}{c}4 \text { studies from } 4 \\
\text { publications } \\
{[43,69-71]}\end{array}$ & $\begin{array}{c}0.85 \text { (95\% CI 0.78, } 0.92 \\
\left.\mathrm{I}^{2}=0 \% ; p \text {-het }=0.92\right)\end{array}$ & $\mathrm{S}$ \\
\hline & & $\begin{array}{l}\text { Dose response: } \\
28 \mathrm{~g} / \text { day } \\
\text { increase }\end{array}$ & total nuts & 11,962 events & $20-95$ & $3-9$ & $\begin{array}{c}4 \text { studies from } 4 \\
\text { publications } \\
{[43,69-71]}\end{array}$ & $\begin{array}{l}0.70 \text { (95\% CI } 0.45,1.08 \\
\left.\mathrm{I}^{2}=69 \% ; p \text {-het }=0.02\right)\end{array}$ & NS \\
\hline & \multirow{3}{*}{$\begin{array}{c}\text { Guo et al. } 2015[63] / 8 \\
\text { prospective studies } \\
\text { from } 8 \text { publications } \\
{[53-56,65,68,70,72]}\end{array}$} & High vs. Low & total nuts & $\begin{array}{l}\text { 30,189 subjects } \\
\text { with } 9554\end{array}$ & $34.6-87.1$ & $4.3-15$ & $\begin{array}{c}3 \text { studies from } 3 \\
\text { publications } \\
{[68,70,72]}\end{array}$ & $\begin{array}{l}0.84 \text { (95\% CI: } 0.76,0.93 \\
\left.p \text {-het }=0.831, \mathrm{I}^{2}=0.0 \%\right)\end{array}$ & $S$ \\
\hline & & \multirow{2}{*}{ Dose-response: } & $\begin{array}{l}\geq 1 \text { serving total } \\
\text { nuts per week }\end{array}$ & $\begin{array}{l}\text { 30,189 subjects } \\
\text { with } 9554\end{array}$ & $34.6-87.1$ & $4.3-15$ & $\begin{array}{c}3 \text { studies from } 3 \\
\text { publications } \\
{[68,70,72]}\end{array}$ & $\begin{array}{l}0.97(95 \% \text { CI } 0.83,1.13 \text {; } \\
\left.p \text {-het }=69.2, \mathrm{I}^{2}=0.039 \%\right)\end{array}$ & NS \\
\hline & & & $\begin{array}{l}\geq 2 \text { servings total } \\
\text { nuts per week }\end{array}$ & $\begin{array}{l}\text { 30,189 subjects } \\
\text { with } 9554\end{array}$ & $34.6-87.1$ & $4.3-15$ & $\begin{array}{c}\text { 3studies from } 3 \\
\text { publications } \\
{[68,70,72]}\end{array}$ & $\begin{array}{l}0.92(95 \% \text { CI } 0.87,0.97 \\
\left.p \text {-het }=0.590, \mathrm{I}^{2}=0.0 \%\right)\end{array}$ & $\mathrm{S}$ \\
\hline
\end{tabular}


Table 2. Cont

\begin{tabular}{|c|c|c|c|c|c|c|c|c|c|}
\hline Variables & Ref/Studies & Analyses & Nut Types & Sample Size & $\begin{array}{c}\text { Sample } \\
\text { Age }\end{array}$ & $\begin{array}{l}\text { Follow-up } \\
\text { Periods }\end{array}$ & $\begin{array}{l}\text { No. of Studies } \\
\text { Included }\end{array}$ & RRs & Effects \\
\hline & $\begin{array}{l}\text { Zhou et al. } 2014 \\
\text { [64]/23 prospective }\end{array}$ & High vs. Low & total nuts & $\begin{array}{c}40,102 \text { subjects } \\
\text { with } 1,2814 \\
\text { events }\end{array}$ & $18-84$ & $4.3-15$ & $\begin{array}{c}4 \text { studies from } 4 \\
\text { publications } \\
{[68,70-72]}\end{array}$ & $\begin{array}{c}0.85(95 \% \text { CI 0.79, } 0.92 ; \\
\left.p \text {-het }=0.927 ; \mathrm{I}^{2}=0.0 \%\right)\end{array}$ & $S$ \\
\hline & $\begin{array}{l}\text { from } 19 \text { publications } \\
{[22,34,38,40,42,43,48,} \\
53-56,68,70-72,86-89]\end{array}$ & $\begin{array}{l}\text { Dose-response: } \\
1 \text { serving/day } \\
\text { increase }\end{array}$ & total nuts & & $18-84$ & $4.3-15$ & $\begin{array}{c}4 \text { studies from } 4 \\
\text { publications } \\
{[68,70-72]}\end{array}$ & $\begin{array}{c}0.66(95 \% \text { CI } 0.44,1.00 \\
\left.p \text {-het }=0.006 ; I^{2}=75.9 \%\right)\end{array}$ & NS \\
\hline \multirow{8}{*}{ Total CHD } & $\begin{array}{l}\text { Bechthold et al. } 2017 \\
\text { [73]/4 prospective } \\
\text { studies from } 4 \\
\text { publications }[37-40]\end{array}$ & High vs. Low & total nuts & $\begin{array}{l}143,934 \text { subjects } \\
\text { with } 5,480 \\
\text { events }\end{array}$ & $30-84$ & $6-26$ & $\begin{array}{c}4 \text { studies from } 4 \\
\text { publications } \\
\text { [37-40] }\end{array}$ & $\begin{array}{l}0.80\left(95 \% \text { CI } 0.62,1.03 ; \mathrm{I}^{2}\right. \\
=79 \%, p \text {-het }=0.002)\end{array}$ & NS \\
\hline & \multirow{6}{*}{$\begin{array}{c}\text { Aune et al. } 2016[14] / 20 \\
\text { prospective studies } \\
\text { from } 29 \text { publications } \\
{[17-29,31-46]}\end{array}$} & \multirow{3}{*}{ High vs. Low } & total nuts & $\begin{array}{c}315,397 \text { subjects } \\
\text { with } 12,331 \\
\text { events }\end{array}$ & $40-80$ & $4-30$ & $\begin{array}{c}11 \text { studies from } 10 \\
\text { publications } \\
{[17-19,22,26-28} \\
37-39]\end{array}$ & $\begin{array}{r}0.76(95 \% \text { CI } 0.69,0.84 ; \\
\left.p \text {-het }=0.04 ; \mathrm{I}^{2}=47.5 \%\right)\end{array}$ & $\mathrm{S}$ \\
\hline & & & peanuts & $\begin{array}{l}265,252 \text { subjects } \\
\text { with } 7025 \\
\text { events }\end{array}$ & $40-79$ & $4-30$ & $\begin{array}{c}5 \text { studies from } 3 \\
\text { publications } \\
{[17-19]}\end{array}$ & $\begin{array}{c}0.76(95 \% \text { CI } 0.69,0.82 ; \\
\left.p \text {-het }=0.65 ; I^{2}=0 \%\right)\end{array}$ & $\mathrm{S}$ \\
\hline & & & tree nuts & $\begin{array}{l}\text { 130,987 subjects } \\
\text { with } 6394 \\
\text { events }\end{array}$ & $55-61$ & $10-30$ & $\begin{array}{c}3 \text { studies from } 2 \\
\text { publications } \\
{[17,19]}\end{array}$ & $\begin{array}{l}0.79(95 \% \text { CI 0.68, 0.92; } \\
\left.p \text {-het = 0.25; I } \mathrm{I}^{2}=28 \%\right)\end{array}$ & $\mathrm{S}$ \\
\hline & & \multirow{3}{*}{$\begin{array}{l}\text { Dose-response: } \\
28 \mathrm{~g} / \text { day } \\
\text { increase in total } \\
\text { nuts, } 10 \mathrm{~g} / \text { day } \\
\text { increase in } \\
\text { peanuts and } \\
\text { tree nuts }\end{array}$} & total nuts & $\begin{array}{l}315,397 \text { subjects } \\
\text { with } 12,331 \\
\text { events }\end{array}$ & $40-80$ & $2-30$ & $\begin{array}{c}11 \text { studies from } 10 \\
\text { publications } \\
{[17-19,22,26-28,} \\
37-39]\end{array}$ & $\begin{array}{c}0.71(95 \% \text { CI } 0.63,0.80 ; \\
\left.p \text {-het }=0.04 ; I^{2}=47.4 \%\right)\end{array}$ & $\mathrm{S}$ \\
\hline & & & peanuts & $\begin{array}{l}265,252 \text { subjects } \\
\text { with } 7025 \\
\text { events }\end{array}$ & $40-79$ & $4-30$ & $\begin{array}{c}5 \text { studies from } 3 \\
\text { publications } \\
\text { [17-19] }\end{array}$ & $\begin{array}{c}0.69(95 \% \text { CI 0.57, } 0.84 ; \\
\left.p \text {-het }=0.12 ; \mathrm{I}^{2}=45.1 \%\right)\end{array}$ & $\mathrm{S}$ \\
\hline & & & tree nuts & $\begin{array}{c}130,987 \text { subjects } \\
\text { with } 6394 \\
\text { events }\end{array}$ & $55-61$ & $10-30$ & $\begin{array}{c}3 \text { studies from } 2 \\
\text { publications } \\
{[17,19]}\end{array}$ & $\begin{array}{l}0.73(95 \% \text { CI } 0.63,0.85 ; \\
\left.p \text {-het }=0.44 ; I^{2}=0 \%\right)\end{array}$ & $\mathrm{S}$ \\
\hline & $\begin{array}{c}\text { Mayhew et al. } 2016 \\
\text { [5]/20 prospective } \\
\text { studies from } 20 \\
\text { publications } \\
{[18-20,22-26,33,37-40} \\
42-44,47-50]\end{array}$ & High vs. Low & total nuts & $\begin{array}{l}\text { 123,971 subjects } \\
\text { with } 4757 \\
\text { events }\end{array}$ & $\geq 24$ & $10-26$ & $\begin{array}{c}3 \text { studies from } 3 \\
\text { publications } \\
{[37,40,90]}\end{array}$ & $\begin{array}{c}0.66(95 \% \text { CI } 0.48-0.91 \\
\left.p \text {-het }=0.0002 ; I^{2}=88 \%\right)\end{array}$ & $\mathrm{S}$ \\
\hline
\end{tabular}


Table 2. Cont.

\begin{tabular}{|c|c|c|c|c|c|c|c|c|c|}
\hline Variables & Ref/Studies & Analyses & Nut Types & Sample Size & $\begin{array}{l}\text { Sample } \\
\text { Age }\end{array}$ & $\begin{array}{l}\text { Follow-up } \\
\text { Periods }\end{array}$ & $\begin{array}{l}\text { No. of Studies } \\
\text { Included }\end{array}$ & RRs & Effects \\
\hline & \multirow{2}{*}{$\begin{array}{c}\text { Weng et al. } 2016[58] / 14 \\
\text { studies from } 10 \\
\text { publications (13 } \\
\text { prospective\&1 RCT) } \\
{[20,25,38-40,47,48,91-93]}\end{array}$} & High vs. Low & total nuts & & $30-80$ & $4.8-26$ & $\begin{array}{c}14 \text { studies from } 10 \\
\text { publications } \\
{[20,25,38-40,47} \\
48,91-93]\end{array}$ & $\begin{array}{l}0.681(95 \% \text { CI } 0.592-0.783 \\
\left.p \text {-het }=0.001 ; I^{2}=62.7 \%\right)\end{array}$ & S \\
\hline & & $\begin{array}{l}\text { Dose-response: } \\
1 \text { serving } \\
(28 \text { g)/week } \\
\text { increase }\end{array}$ & total nuts & & $30-80$ & $4.8-26$ & $\begin{array}{c}13 \text { studies from } 9 \\
\text { publications } \\
{[20,25,38-40,47} \\
48,92,93]\end{array}$ & $\begin{array}{c}0.90(95 \% \text { CI 0.87-0.94; } \\
\left.p \text {-het }=0.000 ; \mathrm{I}^{2}=68.2 \%\right)\end{array}$ & S \\
\hline & \multirow{2}{*}{$\begin{array}{c}\text { Zhou et al. } 2014 \text { [64] } / 23 \\
\text { prospective from } 19 \\
\text { publications } \\
{[22,34,38,40,42,43,48,53-} \\
56,68,70-72,86-89]\end{array}$} & High vs. Low & total nuts & $\begin{array}{l}179,885 \text { subjects } \\
\text { with } 7236 \\
\text { events }\end{array}$ & $16-86$ & $3.8-26$ & $\begin{array}{l}9 \text { studies from } 7 \\
\text { publications [22, } \\
38,40,48,87-89]\end{array}$ & $\begin{array}{c}0.83(95 \% \text { CI } 0.74,0.93 ; \\
\left.p \text {-het }=0.010 ; I^{2}=59.9 \%\right) \\
\text { in risk of CAD }\end{array}$ & S \\
\hline & & $\begin{array}{l}\text { Dose-response: } \\
1 \text { serving ( } 28 \\
\text { g)/day increase }\end{array}$ & total nuts & $\begin{array}{l}179,885 \text { subjects } \\
\text { with } 7236 \\
\text { events }\end{array}$ & $16-86$ & $3.8-26$ & $\begin{array}{l}9 \text { studies from } 7 \\
\text { publications [22, } \\
38,40,48,87-89]\end{array}$ & $\begin{array}{c}0.81(95 \% \text { CI } 0.72,0.91 ; \\
\left.p \text {-het }=0.018 ; I^{2}=56.8 \%\right) \\
\text { in risk of CAD }\end{array}$ & S \\
\hline & \multirow{2}{*}{$\begin{array}{c}\text { Luo et al. } 2014[16] / 18 \\
\text { prospective studies from } \\
18 \text { publications } \\
{[19,20,22,24-26,38-40,42-} \\
44,48,50,53-56]\end{array}$} & High vs. Low & total nuts & 6623 events & $30-84$ & $7-26$ & $\begin{array}{l}6 \text { studies from } 6 \\
\text { publications } \\
{[22,26,38-40,48]}\end{array}$ & $\begin{array}{c}0.66(95 \% \text { CI } 0.55,0.78 \\
\left.p \text {-het }=0.02 ; I^{2}=62.5 \%\right)\end{array}$ & $S$ \\
\hline & & $\begin{array}{l}\text { Dose-response: } \\
1 \text { serving ( } 28 \\
\text { g)/day increase }\end{array}$ & total nuts & 6623 events & $30-84$ & $7-26$ & $\begin{array}{l}6 \text { studies from } 6 \\
\text { publications } \\
{[22,26,38-40,48]}\end{array}$ & $\begin{array}{r}0.72(95 \% \text { CI } 0.64,0.81 \\
\left.p \text {-het }=0.644 ; \mathrm{I}^{2}=0.0 \%\right)\end{array}$ & $\mathrm{S}$ \\
\hline & \multirow{5}{*}{$\begin{array}{c}\text { Ma et al. } 2014[74] \text { in } 13 \\
\text { prospective studies from } \\
9 \text { publications } \\
{[20,25,38-40,47,48,92,93]}\end{array}$} & High vs. Low & total nuts & $\begin{array}{l}493,081 \text { subjects } \\
\text { with } 6127 \\
\text { events }\end{array}$ & $34-84$ & $4.8-26$ & $\begin{array}{c}13 \text { prospective } \\
\text { studies from } 9 \\
\text { publications } \\
{[20,25,38-40,47} \\
48,92,93]\end{array}$ & $\begin{array}{c}0.660(95 \% \text { CI } 0.581,0.748 \\
\left.\mathrm{I}^{2}=39.6 \%\right) \text { in risk } \\
\text { of CAD }\end{array}$ & $\mathrm{S}$ \\
\hline & & \multirow{4}{*}{ Dose response } & $\begin{array}{c}1 \\
\text { serving/week } \\
\text { of nut intake }\end{array}$ & \multirow{4}{*}{$\begin{array}{l}236,008 \text { subjects } \\
\text { with } 4886 \\
\text { events }\end{array}$} & \multirow{4}{*}{$34-80$} & \multirow{4}{*}{$4.8-22$} & \multirow{4}{*}{$\begin{array}{l}7 \text { studies from } 4 \\
\text { publications } \\
{[25,38,47,48]}\end{array}$} & $\begin{array}{c}\text { in risk of CAD: } 0.96(95 \% \\
\text { CI } 0.89,1.02)\end{array}$ & NS \\
\hline & & & $\begin{array}{c}\text { for } 2 \\
\text { servings/week } \\
\text { of nut intake }\end{array}$ & & & & & $0.91(95 \%$ CI $0.82,0.99)$ & $\mathrm{S}$ \\
\hline & & & $\begin{array}{c}3 \\
\text { servings/week } \\
\text { of nut intake }\end{array}$ & & & & & $0.85(95 \%$ CI $0.77,0.95)$ & S \\
\hline & & & $\begin{array}{c}4 \\
\text { servings/week } \\
\text { of nut intake }\end{array}$ & & & & & $0.80(95 \%$ CI $0.72,0.89)$ & S \\
\hline
\end{tabular}


Table 2. Cont

\begin{tabular}{|c|c|c|c|c|c|c|c|c|c|}
\hline Variables & Ref/Studies & Analyses & Nut Types & Sample Size & $\begin{array}{c}\text { Sample } \\
\text { Age }\end{array}$ & $\begin{array}{l}\text { Follow-up } \\
\text { Periods }\end{array}$ & $\begin{array}{l}\text { No. of Studies } \\
\text { Included }\end{array}$ & RRs & Effects \\
\hline & & & $\begin{array}{c}5 \\
\text { servings/week } \\
\text { of nut intake }\end{array}$ & & & & & $0.75(95 \%$ CI $0.65,0.85)$ & $\mathrm{S}$ \\
\hline & & & $\begin{array}{c}6 \\
\text { servings/week } \\
\text { of nut intake }\end{array}$ & & & & & $0.70(95 \%$ CI $0.58,0.83)$ & S \\
\hline \multirow{4}{*}{$\begin{array}{l}\text { Non-fatalCHD/ } \\
\text { fatal CHD }\end{array}$} & \multirow{2}{*}{$\begin{array}{c}\text { Mayhew et al. } 2016 \\
\text { [5]/20 prospective } \\
\text { studies from } 20 \\
\text { publications }[18-20,22- \\
26,33,37-40,42-44,47-50]\end{array}$} & High vs. Low & total nuts & $\begin{array}{l}138,678 \text { subjects } \\
\text { with } 1565 \\
\text { events }\end{array}$ & $34-84$ & $14-17$ & $\begin{array}{c}3 \text { studies from } 3 \\
\text { publications } \\
{[38,39,47]}\end{array}$ & $\begin{array}{l}\text { Non-fatal CHD: } 0.71(95 \% \\
\text { CI 0.49-1.03; } p \text {-het = } 0.03 \text {; } \\
\left.\qquad \mathrm{I}^{2}=72 \%\right)\end{array}$ & NS \\
\hline & & $\begin{array}{l}\text { Dose response: } \\
4 \\
\text { servings/week } \\
\text { increase }\end{array}$ & total nuts & $\begin{array}{l}138,678 \text { subjects } \\
\text { with } 1565 \\
\text { events }\end{array}$ & $34-84$ & $14-17$ & $\begin{array}{c}3 \text { studies from } 3 \\
\text { publications } \\
{[38,39,47]}\end{array}$ & $\begin{array}{c}\text { Non-fatal CHD: } 0.81 \text { (95\% } \\
\text { CI 0.72-0.96) }\end{array}$ & S \\
\hline & \multirow{2}{*}{$\begin{array}{c}\text { Afshin et al. } 2014[57] / 16 \\
\text { studies }(14 \text { prospective } \\
{[2,19,22,38-40,42,43,47} \\
53,55,56,65,85,86] \& 2 \\
\text { RCTs }[2,66]\end{array}$} & \multirow{2}{*}{$\begin{array}{c}\text { Dose response: } \\
4 \text { servings } \\
(28.4 \mathrm{~g}) / \text { week }\end{array}$} & \multirow[t]{2}{*}{ total nuts } & $\begin{array}{l}\text { 141,390 subjects } \\
\text { with } 2101 \\
\text { events }\end{array}$ & $34-84$ & $14-17$ & $\begin{array}{c}4 \text { studies from } 4 \\
\text { publications } \\
{[2,38,39,47]}\end{array}$ & $\begin{array}{c}\text { Non-fatal CHD: } 0.78 \text { (95\% } \\
\text { CI 0.67-0.92; } \\
\left.p \text {-het }=0.463 ; \mathrm{I}^{2}=0.0 \%\right)\end{array}$ & $\mathrm{S}$ \\
\hline & & & & $\begin{array}{l}\text { 206,114 subjects } \\
\text { with } 6749 \\
\text { events }\end{array}$ & $40-84$ & $17-30$ & $\begin{array}{c}6 \text { studies from } 5 \\
\text { publications } \\
{[2,19,22,38,39]}\end{array}$ & $\begin{array}{c}\text { Fatal CHD: } 0.76(95 \% \text { CI } \\
0.69-0.84 ; p \text {-het }=0.227 \\
\left.I^{2}=27.7 \%\right)\end{array}$ & $\mathrm{S}$ \\
\hline \multirow{5}{*}{ Total stroke } & \multirow{2}{*}{$\begin{array}{l}\text { Bechthold et al. } 2017 \\
\text { [73]/4 prospective } \\
\text { studies from } 4 \\
\text { publications [37-40] }\end{array}$} & High vs. Low & total nuts & 7490 events & $30-84$ & $17-26$ & $\begin{array}{c}6 \text { studies from } 5 \\
\text { publications } \\
{[33,41-44]}\end{array}$ & $\begin{array}{l}0.94(95 \% \text { CI 0.85, 1.05, } \\
\left.\mathrm{I}^{2}=18 \%, p \text {-het }=0.30\right)\end{array}$ & NS \\
\hline & & $\begin{array}{l}\text { Dose response: } \\
28 \mathrm{~g} / \text { day } \\
\text { increase }\end{array}$ & total nuts & 7490 events & $30-84$ & $17-26$ & $\begin{array}{c}6 \text { studies from } 5 \\
\text { publications } \\
{[33,41-44]}\end{array}$ & $\begin{array}{l}0.99(95 \% \text { CI 0.84, 1.17, } \\
\left.\mathrm{I}^{2}=45 \%, p \text {-het }=0.11\right)\end{array}$ & NS \\
\hline & \multirow{3}{*}{$\begin{array}{c}\text { Aune et al. } 2016[14] / 20 \\
\text { prospective studies from } \\
29 \text { publications } \\
{[17-29,31-46]}\end{array}$} & \multirow{3}{*}{ High vs. Low } & total nuts & $\begin{array}{l}396,768 \text { subjects } \\
\text { with } 9272 \\
\text { events }\end{array}$ & $40-86.7$ & $4-26$ & $\begin{array}{c}10 \text { studies from } 9 \\
\text { publications } \\
{[17,18,27,28,33,34,} \\
41-43]\end{array}$ & $\begin{array}{c}0.89(95 \% \text { CI } 0.82,0.97 \\
\left.p \text {-het }=0.90 ; \mathrm{I}^{2}=0 \%\right)\end{array}$ & $\mathrm{S}$ \\
\hline & & & peanuts & $\begin{array}{l}265,252 \text { subjects } \\
\text { with } 3315 \\
\text { events. }\end{array}$ & $40-79$ & $4-30$ & $\begin{array}{c}5 \text { studies from } 3 \\
\text { publications } \\
{[18,19,50]}\end{array}$ & $\begin{array}{l}0.83(95 \% \text { CI } 0.69-1.00 ; \\
\left.p \text {-het }=0.12 ; I^{2}=45.9 \%\right)\end{array}$ & NS \\
\hline & & & tree nuts & $\begin{array}{l}\text { 130,987 subjects } \\
\text { with } 2130 \\
\text { events }\end{array}$ & $55-61$ & $10-30$ & $\begin{array}{c}3 \text { studies from } 2 \\
\text { publications } \\
{[17,19]}\end{array}$ & $\begin{array}{c}0.93(95 \% \text { CI } 0.77,1.13 ; \\
\left.p \text {-het }=0.44 ; \mathrm{I}^{2}=0 \%\right)\end{array}$ & NS \\
\hline
\end{tabular}


Table 2. Cont.

\begin{tabular}{|c|c|c|c|c|c|c|c|c|c|}
\hline Variables & Ref/Studies & Analyses & Nut Types & Sample Size & $\begin{array}{l}\text { Sample } \\
\text { Age }\end{array}$ & $\begin{array}{l}\text { Follow-up } \\
\text { Periods }\end{array}$ & $\begin{array}{l}\text { No. of Studies } \\
\text { Included }\end{array}$ & RRs & Effects \\
\hline & & \multirow{3}{*}{$\begin{array}{l}\text { Dose response: } \\
28 \mathrm{~g} / \text { day } \\
\text { increase in total } \\
\text { nuts, } 10 \mathrm{~g} / \text { day } \\
\text { increase in } \\
\text { peanuts and } \\
\text { tree nuts }\end{array}$} & total nuts & $\begin{array}{l}\text { 396,768 subjects } \\
\text { with } 9272 \\
\text { events }\end{array}$ & $30-86.7$ & $4-26$ & $\begin{array}{c}11 \text { studies from } 9 \\
\text { publications } \\
{[17,18,27,28,33,34,} \\
41-43]\end{array}$ & $\begin{array}{r}0.93(95 \% \text { CI 0.83, 1.05; } \\
\left.p \text {-het = 0.31; } \mathrm{I}^{2}=13.7 \%\right)\end{array}$ & NS \\
\hline & & & peanuts & $\begin{array}{l}265,252 \text { subjects } \\
\text { with } 3315 \\
\text { events }\end{array}$ & $40-79$ & $4-30$ & $\begin{array}{c}5 \text { studies from } 3 \\
\text { publications } \\
\text { [17-19] }\end{array}$ & $\begin{array}{c}0.63(95 \% \text { CI } 0.41,0.95 \\
\left.p \text {-het }=0.001 ; I^{2}=77.6 \%\right)\end{array}$ & S \\
\hline & & & tree nuts & $\begin{array}{l}\text { 130,987 subjects } \\
\text { with } 2130 \\
\text { events }\end{array}$ & $55-69$ & $10-30$ & $\begin{array}{c}3 \text { studies from } 2 \\
\text { publications } \\
{[19,50]}\end{array}$ & $\begin{array}{l}0.89(95 \% \text { CI } 0.69,1.14 \\
\left.p \text {-het }=0.58 ; I^{2}=0 \%\right)\end{array}$ & NS \\
\hline & $\begin{array}{l}\text { Mayhew et al. } 2016 \\
\text { [5]/20 prospective } \\
\text { studies from } 20 \\
\text { publications }[18-20,22- \\
26,33,37-40,42-44,47-50]\end{array}$ & High vs. Low & total nuts & $\begin{array}{l}157,826 \text { subjects } \\
\text { with } 4318 \\
\text { events }\end{array}$ & $30-75$ & $4-26$ & $\begin{array}{c}2 \text { studies from } 2 \\
\text { publications } \\
{[33,42]}\end{array}$ & $\begin{array}{l}1.05(95 \% \text { CI } 0.69-1.61 ; \\
\left.p \text {-het = 0.04; } I^{2}=77 \%\right)\end{array}$ & NS \\
\hline & $\begin{array}{c}\text { Afshin et al. } 2014[57] / 16 \\
\text { studies }(14 \text { prospective } \\
{[2,19,22,38-40,42,43,47} \\
53,55,56,65,85,86] \& 2 \\
\text { RCTs }[2,66]\end{array}$ & $\begin{array}{l}\text { Dose response: } \\
\text { for } 4 \text { servings } \\
(28.4 \mathrm{~g}) / \text { week }\end{array}$ & total nuts & $\begin{array}{l}155,685 \text { subjects } \\
\text { with } 5544 \\
\text { events }\end{array}$ & $30-86.7$ & $4.8-26$ & $\begin{array}{c}4 \text { studies from } 3 \\
\text { publications } \\
{[2,42,43]}\end{array}$ & $\begin{array}{c}0.89(95 \% \text { CI } 0.74,1.05 \\
\left.p \text {-het }=0.012 ; I^{2}=72.7 \%\right)\end{array}$ & NS \\
\hline & \multirow{2}{*}{$\begin{array}{c}\text { Zhou et al. } 2014[64] / 23 \\
\text { prospective from } 19 \\
\text { publications } \\
{[22,34,38,40,42,43,48,53-} \\
56,68,70-72,86-89]\end{array}$} & High vs. Low & total nuts & $\begin{array}{l}\text { 182,730 subjects } \\
\text { with } 5669 \\
\text { events }\end{array}$ & $30-86.7$ & $9-26$ & $\begin{array}{l}4 \text { studies from } 3 \\
\text { publications } \\
{[34,42,43]}\end{array}$ & $\begin{array}{c}0.87(95 \% \text { CI 0.74, 1.03; } \\
\left.p \text {-het }=0.317 ; I^{2}=15.0 \%\right)\end{array}$ & NS \\
\hline & & $\begin{array}{l}\text { Dose response: } \\
28 \text { g/day } \\
\text { increase }\end{array}$ & total nuts & $\begin{array}{l}\text { 182,730 subjects } \\
\text { with } 5669 \\
\text { events }\end{array}$ & $30-86.7$ & $9-26$ & $\begin{array}{l}4 \text { studies from } 3 \\
\text { publications } \\
{[34,42,43]}\end{array}$ & $\begin{array}{c}0.90(95 \% \text { CI } 0.71,1.14 ; \\
\left.p \text {-het }=0.114 ; I^{2}=49.6 \%\right)\end{array}$ & NS \\
\hline & $\begin{array}{c}\text { Luo et al. } 2014[16] / 18 \\
\text { prospective studies from } \\
18 \text { publications } \\
{[19,20,22,24-26,38-40,42-} \\
44,48,50,53-56]\end{array}$ & $\begin{array}{l}\text { High }(\geq 2 \text { to } \geq 7 \\
\text { servings / week }) \\
\text { vs. Low ( } 0 \text { to } \leq 1 \\
\text { serving/week) }\end{array}$ & total nuts & 6487 events & $30-86.7$ & $21-26$ & $\begin{array}{c}3 \text { studies from } 3 \\
\text { publications } \\
\text { [42-44] }\end{array}$ & $\begin{array}{c}0.91(95 \% \text { CI } 0.81,1.02 \\
\left.p \text {-het }=0.285 ; I^{2}=20.4 \%\right)\end{array}$ & NS \\
\hline & $\begin{array}{l}\text { Shi et al. } 2014[75] \text { in } 4 \\
\text { prospective studies from } \\
3 \text { publications }[34,42,43]\end{array}$ & High vs. Low & total nuts & $\begin{array}{l}468,887 \text { subjects } \\
\text { with } 10,493 \\
\text { events }\end{array}$ & $30-75$ & $9-26$ & $\begin{array}{c}3 \text { publications } \\
{[34,42,43]}\end{array}$ & $\begin{array}{c}0.90(95 \% \text { CI } 0.81,0.99 \\
\left.p \text {-het }=0.527, \mathrm{I}^{2}=0\right)\end{array}$ & $\mathrm{S}$ \\
\hline
\end{tabular}

CAD, coronary artery disease; CI, confidence interval; CVD, cardiovascular disease; d, day; HD, coronary heart disease; het, heterogeneity; NS, non-significant; RCT, randomized-controlled trial; Ref, references; RR, relative risk; $\mathrm{S}$, significant. 
3.2. Weight, Blood Glucose, Lipids and Inflammatory Markers, Endothelial Function and Blood Pressure in Meta-Analyses of Intervention Studies

\subsubsection{Body Weight}

A summary of nut consumption and anthropometric parameters in RCTs is shown in Table 3. Forest plots for Table 3 are presented in Supplementary Figures S22-S24.

In a recent meta-analysis of RCTs by Guasch-Ferre et al. 2018 [94], a diet rich in walnuts did not alter weight [weighted mean difference $(\mathrm{WMD})=-0.12 \mathrm{~kg} ; 95 \% \mathrm{CI}-2.12,1.88 ; p=0.90$ ] and body mass index (BMI) $\left(\mathrm{WMD}=-0.11 \mathrm{~kg} / \mathrm{m}^{2} ; 95 \% \mathrm{CI}-1.15,0.92 ; p=0.82\right)$ compared with control diets in 10 studies from 10 publications [95-104] and 6 studies from 6 publications [95-97,105-107] respectively.

Perna et al. 2016 [108] conducted a meta-analysis of 3 studies from 3 publications [109-111] using a Bayesian random effect meta-analysis of mean differences from baseline across treatments (MD $\Delta$ ) (e.g., a diet supplemented with hazelnuts vs. a control diet). They found that BMI [pooled MD $\Delta=0.062 \mathrm{~kg} / \mathrm{m}^{2}$; $95 \%$ highest posterior density interval $(\mathrm{HPD})=-0.293 ; 0.469]$ remained unchanged following hazelnut consumption (29-69 g/day; average $45 \mathrm{~g}$ /day) for 28-84 days (average 56 days). This meta-analysis was too small to provide accurate data and other metanalyses found no effect.

Banel et al. 2009 [112] conducted a meta-analysis of 13 studies (13 publications) [96,97,101-104, 113-119]. Both BMI [mean difference $(\mathrm{MD})=-0.4 \mathrm{~kg} / \mathrm{m}^{2} ; p=0.5$ ] and body weight $(\mathrm{MD}=-0.05 \mathrm{~kg}$; $p=0.97)$ remained unchanged compared with control diets. Publication bias was not observed [112].

Flores-Mateo et al. 2013 [120] undertook a meta-analysis of 33 RCTs published prior to December 2012 to investigate the effects of nut consumption on adiposity in comparison with control diets. The findings showed that nut consumption did not significantly affect body weight $(\mathrm{MD}=-0.47 \mathrm{~kg})$, BMI $\left(\mathrm{MD}=-0.40 \mathrm{~kg} / \mathrm{m}^{2}\right)$ and waist circumference $(\mathrm{MD}=-1.25 \mathrm{~cm})$ in comparison with control diets. The analysis of body weight [96,97,101-104,121-140], BMI [95-97,107,114,121,125,127,131,136,137, 140-143] and waist circumference [95,107,125,131,132] came from 26, 15 and 5 publications respectively. Publication bias was not detected in analyses [120].

Mejia et al. 2014 [144] undertook a meta-analysis to examine the effect of tree nuts on waist circumference (15 studies; $n=1050$ ) from 15 publications including healthy subjects [109], subjects with dyslipidemia [122], metabolic syndrome [99,105,107,132,137,145-148] and T2DM [95,125,149,150]. Tree nut consumption did not significantly alter waist circumference compared with a control diet.

None of the meta-analyses showed significant changes in body weight, waist circumference and BMI. The concerns that nut consumption may lead to body weight gain caused by the high-energy density with a high-fat content may be allayed. However, a recent meta-analysis [77] showed significant positive effects of nut consumption on body weight, waist circumference and BMI compared with a control diet. A meta-analysis [77] of 56 intervention studies (55 publications) [95,96,99-105,110,111,121, 122,124-129,132-140,146,147,151-175] showed a significant reduction in body weight (WMD = -0.22 kg; $95 \%$ CI $\left.-0.40,-0.04 ; \mathrm{I}^{2}=75 \%\right)$. Significant reductions in waist circumference $(-0.51 \mathrm{~cm} ; 95 \%$ CI $\left.-0.95,-0.07 ; \mathrm{I}^{2}=82 \%\right)$ and BMI $\left(-0.16 \mathrm{~kg} / \mathrm{m}^{2} ; 95 \% \mathrm{CI}-0.31,-0.01 ; \mathrm{I}^{2}=76 \%\right)$ have been shown in a data from 23 intervention studies (22 publications) [95,99,105,107,125,132,137,138,146, $147,151,153,154,156,159,161,163,164,169,172,173,176]$ and 39 intervention studies (38 publications) [95, $96,105,107,110,111,114,121,125,127,136-138,140-143,146,151-156,158,162-166,169,171-177]$, respectively. In this meta-analysis, eleven interventions [99,125,139,156,161,166-171] were weight interventions with nuts. Several interventions [111,128,132,135,174] and a prospective study [153] and non-randomized study [121] reported weight reduction during an intervention without a weight loss program. It is also noted that this meta-analysis included two prospective studies [125,153].

The effects of nut consumption on glycemic control, lipid profiles and blood pressure from meta-analyses of intervention studies are summarized in Table 4 . The summary of the effects of nut consumption on inflammation in meta-analyses of intervention studies is shown in Table 5. The effect of nut consumption on endothelial function from meta-analyses of intervention studies is summarized in Table 6. Forest plots for Tables 4-6 are presented in Supplementary Figures S25-S42. 
Table 3. Summary of nut consumption and anthropometric parameters in intervention studies.

\begin{tabular}{|c|c|c|c|c|c|c|}
\hline \multicolumn{7}{|c|}{ References Comparison } \\
\hline Variables & $\begin{array}{l}\text { Li et al. } 2018 \text { [77]: nuts } \\
\text { vs. controls }\end{array}$ & $\begin{array}{l}\text { Guasch-Ferre et al. } \\
2018 \text { [94]: walnuts vs. } \\
\text { controls }\end{array}$ & $\begin{array}{c}\text { Perna et al. } 2016 \text { [108]: } \\
\text { Bayesian meta-analysis } \\
\text { of hazelnuts }\end{array}$ & $\begin{array}{l}\text { Mejia et al. } 2014 \text { [144]: } \\
\sim 50 \text { g/ day of tree nuts } \\
\text { over } \sim 8 \text { weeks vs. } \\
\text { controls }\end{array}$ & $\begin{array}{l}\text { Flores-Mateo et al. } 2013 \\
\text { [120]: nuts vs. controls }\end{array}$ & $\begin{array}{l}\text { Banel et al. } 2009 \text { [112]: } \\
\text { walnuts vs. controls }\end{array}$ \\
\hline BW & $\begin{array}{c}\text { WMD }=-0.22 \mathrm{~kg} \\
(95 \% \text { CI }-0.40,-0.04) \\
\text { From 56 RCTs } \\
(55 \text { publications }) \\
{[95,96,99-105,110,111,} \\
121,122,124-129,132- \\
140,146,147,151-175]\end{array}$ & $\begin{array}{c}\text { WMD }=-0.12 \mathrm{~kg} \\
(95 \% \text { CI }-2.12,1.88 ; \\
p \text { overall effect }=0.90) \\
\text { From 10 RCTs } \\
\text { [95-104] }\end{array}$ & & & $\begin{array}{c}\text { WMD }=-0.47 \mathrm{~kg}(95 \% \\
\text { CI }-1.17,0.22 ; \mathrm{I}^{2}=7 \% ; \\
p \text { overall effect }=\mathrm{NS}) \\
\text { From } 26 \mathrm{RCTs} \\
{[96,97,101-104,121-140]}\end{array}$ & $\begin{array}{c}\text { WMD }=-0.05 \mathrm{~kg} \\
\text { ( } p \text { overall effect }=0.97) \\
\text { No } 95 \% \text { CI reported. } \\
\text { From 13 RCTs } \\
{[96,97,101-104,113-119]}\end{array}$ \\
\hline WC & $\begin{array}{c}\text { WMD }=-0.51 \mathrm{~cm} \\
(95 \% \text { CI }-0.95,-0.07) \\
\text { From } 23 \text { RCTs } \\
(22 \text { publications }) \\
{[95,99,105,107,125,132,} \\
137,138,146,147,151,153, \\
154,156,159,161,163,164, \\
169,172,173,176]\end{array}$ & & & $\begin{array}{c}\mathrm{MD}=-0.62 \mathrm{~cm}(95 \% \\
\mathrm{CI}-1.54,0.30 ; \mathrm{I}^{2}=67 \% ; \\
p \text {-het }<0.0001 ; p \text { overall } \\
\text { effect }=0.19) \\
\text { From } 15 \text { RCTs } \\
{[95,99,105,107,109,122} \\
125,132,137,145-150]\end{array}$ & $\begin{array}{c}\text { WMD }=-1.25 \mathrm{~cm}(95 \% \\
\mathrm{CI}-2.82,0.31 ; \mathrm{I}^{2}=28 \% \text {; } \\
p \text { overall effect }=\mathrm{NS}) \\
\text { From } 5 \text { RCTs } \\
{[95,107,125,131,132]}\end{array}$ & \\
\hline BMI & $\begin{array}{c}\text { WMD }=-0.16 \mathrm{~kg} / \mathrm{m}^{2} \\
(95 \% \text { CI }-0.31,-0.01) \\
\text { From 39 RCTs } \\
\text { (38 publications) }[95,96, \\
105,107,110,111,114,121, \\
125,127,136-138,140- \\
143,146,151-156,158, \\
162-166,169,171-177]\end{array}$ & $\begin{array}{c}\text { WMD }=-0.11 \mathrm{~kg} / \mathrm{m}^{2} \\
(95 \% \mathrm{CI}-1.15,0.92 ; p \\
\text { overall effect }=0.82) \\
\text { From } 6 \text { RCTs } \\
{[95-97,105,107,114]}\end{array}$ & $\begin{array}{c}\mathrm{MD} \Delta=0.062 \mathrm{~kg} / \mathrm{m}^{2} \\
(95 \% \mathrm{HPD}-0.293 \\
0.469) \\
\text { From } 3 \text { RTCs }[109-111]\end{array}$ & & $\begin{array}{c}\text { WMD }=-0.40 \mathrm{~kg} / \mathrm{m}^{2} \\
(95 \% \text { CI }-0.97,0.17 \\
\mathrm{I}^{2}=49 \% ; p \text { overall } \\
\text { effect = NS) } \\
\text { From } 15 \text { RCTs }[95-97 \\
107,114,121,125,127,131 \\
136,137,140-143]\end{array}$ & $\begin{array}{c}\text { WMD }=-0.4 \mathrm{~kg} / \mathrm{m}^{2} \\
(p \text { overall effect }=0.5) \\
\text { No } 95 \% \text { CI reported. } \\
\text { From } 13 \text { RCTs } \\
{[96,97,101-104,113-119]}\end{array}$ \\
\hline
\end{tabular}

BW body weight, BMI, body mass index; het, heterogeneity; $95 \% \mathrm{HPD}, 95 \%$ highest posterior density interval; MD $\Delta$, mean differences of Delta-changes from baseline across treatment; NS, non-significant; T2DM, type 2 diabetes mellitus; WC, waist circumference, WMD; weighted mean difference. 
Table 4. Summary of nut consumption and glucose, insulin, lipids and blood pressure in intervention studies.

\begin{tabular}{|c|c|c|c|c|c|c|c|c|c|c|c|}
\hline \multicolumn{12}{|c|}{ References Comparison } \\
\hline Variables & $\begin{array}{l}\text { Guasch-Ferre } \\
\text { et al. 2018 [94]: } \\
\text { walnuts vs. } \\
\text { controls }\end{array}$ & $\begin{array}{l}\text { Mazidi et al. } \\
\text { 2016 [178]: } \\
\text { nuts vs. } \\
\text { controls }\end{array}$ & $\begin{array}{l}\text { Musa-Veloso } \\
\text { et al. } 2016 \text { [179]: } \\
\text { almonds vs. } \\
\text { controls }\end{array}$ & $\begin{array}{l}\text { Perna et al. } 2016 \\
\text { [108]: Bayesian } \\
\text { meta-analysis of } \\
\text { hazelnuts }\end{array}$ & $\begin{array}{l}\text { Gobbo et al. } 2015 \\
\text { [180]: per } 1 \\
\text { serving/day of } \\
\text { tree nuts vs. } \\
\text { controls }\end{array}$ & $\begin{array}{l}\text { Mohammadifard } \\
\text { et al. } 2015 \text { [181]: } \\
\text { nuts vs. controls }\end{array}$ & $\begin{array}{c}\text { Mejia et al. } 2014 \\
\text { [144]: } \sim 50 \mathrm{~g} / \text { day } \\
\text { of tree nuts over } \\
\sim 8 \text { weeks vs. } \\
\text { controls }\end{array}$ & $\begin{array}{l}\text { Viguiliouk et al. } \\
2014 \text { [3]: tree } \\
\text { nuts vs. controls } \\
\text { in subjects with } \\
\text { T2DM }\end{array}$ & $\begin{array}{l}\text { Sabate et al } \\
2010 \text { [182]: } 67 \\
\text { g/day of nuts } \\
\text { vs. controls }\end{array}$ & $\begin{array}{c}\text { Phung et al. } \\
\text { 2009 [183]: } \\
\text { almonds } \\
\text { (25-169 g/day) } \\
\text { vs. controls } \\
\end{array}$ & $\begin{array}{l}\text { Banel et al. } \\
2009 \text { [112]: } \\
\text { walnuts vs. } \\
\text { controls }\end{array}$ \\
\hline FBG & & $\begin{array}{l}-1.45 \mathrm{mg} / \mathrm{dL} \\
(95 \% \mathrm{CI}-2.20, \\
0.70 ; p \text { overall } \\
\text { effect }<0.05) \\
=-0.08 \mathrm{mmol} / \mathrm{L} \\
(95 \% \mathrm{CI}-0.12 \\
0.039 \mathrm{mmol} / \mathrm{L})\end{array}$ & & & & & $\begin{array}{c}-0.08 \mathrm{mmol} / \mathrm{L} \\
(95 \% \mathrm{CI}-0.16 \\
-0.01 ; \mathrm{I}^{2}=41 \% \\
p \text {-het }=0.02 \\
p \text { overall effect }= \\
0.03)\end{array}$ & $\begin{array}{c}-0.15 \mathrm{mmol} / \mathrm{L} \\
(95 \% \mathrm{CI}-0.27 \\
-0.02 ; \mathrm{I}^{2}=35 \% \\
p \text {-het }=0.12 \\
p \text { overall effect }= \\
0.03)\end{array}$ & & & \\
\hline $\begin{array}{l}\text { Fasting } \\
\text { Insulin }\end{array}$ & & & & & & & & $\begin{array}{c}-3.42 \mathrm{pmol} / \mathrm{L} \\
(95 \% \mathrm{CI}-10.06 \\
3.21 ; \mathrm{I}^{2}=72 \% \\
p \text {-het }=0.0004 \\
p \text { overall effect }= \\
0.031)\end{array}$ & & & \\
\hline HOMA-IR & & & & & & & & $\begin{array}{c}-0.24(95 \% \text { CI } \\
-0.51,0.04 ; \\
\mathrm{I}^{2}=87 \% \\
p \text {-het = } 0.0005 ; \\
p \text { overall effect }= \\
0.10)\end{array}$ & & & \\
\hline $\mathrm{HbA1c}$ & & & & & & & & $\begin{array}{c}-0.07 \%(-95 \% \\
\text { CI } 0.10,-0.03 \\
\mathrm{I}^{2}=37 \% \\
p \text {-het }=0.13 \\
p \text { overall effect }= \\
0.0003)\end{array}$ & & & \\
\hline TC & $\begin{array}{c}-6.99 \mathrm{mg} / \mathrm{dL} ; \\
(95 \% \mathrm{CI}-9.39 \\
-4.58 \mathrm{mg} / \mathrm{dL} ; \\
p \text { overall effect } \\
<0.001 ; \\
\mathrm{I}^{2}=0.0 \% \\
p \text {-het }=0.64) \\
=-0.18 \mathrm{mmol} / \mathrm{L} \\
(95 \% \mathrm{CI}-0.24 \\
-0.118 \mathrm{mmol} / \mathrm{L})\end{array}$ & $\begin{array}{c}-0.82 \mathrm{mg} / \mathrm{dL} \\
(95 \% \mathrm{CI} \\
-1.53 \mathrm{mg} / \mathrm{dL} \\
-0.11 ; \\
p \text { overall effect } \\
<0.05)= \\
-0.021 \mathrm{mmol} / \mathrm{L} \\
(95 \% \mathrm{CI}-0.04 \\
-0.003 \mathrm{mmol} / \mathrm{L})\end{array}$ & $\begin{array}{c}-0.153 \mathrm{mmol} / \mathrm{L} \\
(95 \% \mathrm{CI}-0.235 \\
-0.070 \mathrm{mmol} / \mathrm{L} ; \\
p \text { overall effect }< \\
0.001)\end{array}$ & $\begin{array}{c}\mathrm{MD} \Delta=-0.127 \\
\mathrm{mmol} / \mathrm{L}(95 \% \\
\mathrm{HPD}-0.284 \\
0.014 \mathrm{mmol} / \mathrm{L})\end{array}$ & $\begin{array}{c}-4.7 \mathrm{mg} / \mathrm{dL} \\
(95 \% \mathrm{CI}-5.3 \\
-4.0 \mathrm{mg} / \mathrm{dL} ; \\
\mathrm{I}^{2}>30 \% ; p \text {-het }= \\
0.001 ; p \text { overall } \\
\text { effect }<0.05) \\
=-0.12 \mathrm{mmol} / \mathrm{L} \\
(95 \% \mathrm{CI}-0.14 \\
-0.1 \mathrm{mmol} / \mathrm{L})\end{array}$ & & & & $\begin{array}{c}-10.9 \mathrm{mg} / \mathrm{dL} \\
(95 \% \mathrm{CI}-14.1, \\
-7.8 \mathrm{mg} / \mathrm{dL}) \\
(5.1 \% \text { change; } \\
p<0.001) \\
=-0.28 \\
\mathrm{mmol} / \mathrm{L}(95 \% \\
C \mathrm{CI}-0.36 \\
-0.2 \mathrm{mmol} / \mathrm{L})\end{array}$ & $\begin{array}{l}-0.18 \mathrm{mmol} / \mathrm{L} \\
(95 \% \mathrm{CI}-0.34, \\
-0.02 \mathrm{mmol} / \mathrm{L} ; \\
\mathrm{I}^{2}=0.0 \% ; p \text {-het } \\
=\mathrm{NS} ; p \text { overall } \\
\text { effect }=0.030)\end{array}$ & $\begin{array}{c}-10.3 \mathrm{mg} / \mathrm{dL} \\
(95 \% \mathrm{CI} \\
-14.76, \\
-5.83 \mathrm{mg} / \mathrm{dL} ; \\
\mathrm{I}^{2}=0.0 \% ; p \text {-het } \\
=0.63 ; p \\
\text { overall effect < } \\
0.001) \\
= \\
-0.26 \mathrm{mmol} / \mathrm{L} \\
(95 \% \mathrm{CI}-0.38, \\
-0.15 \mathrm{mmol} / \mathrm{L})\end{array}$ \\
\hline
\end{tabular}


Table 4. Cont.

\begin{tabular}{|c|c|c|c|c|c|c|c|c|c|c|c|}
\hline \multicolumn{12}{|c|}{ References Comparison } \\
\hline Variables & $\begin{array}{l}\text { Guasch-Ferre } \\
\text { et al. } 2018 \text { [94]: } \\
\text { walnuts vs. } \\
\text { controls }\end{array}$ & $\begin{array}{l}\text { Mazidi et al. } \\
\text { 2016 [178]: } \\
\text { nuts vs. } \\
\text { controls }\end{array}$ & $\begin{array}{l}\text { Musa-Veloso et } \\
\text { al. } 2016 \text { [179]: } \\
\text { almonds vs. } \\
\text { controls }\end{array}$ & $\begin{array}{l}\text { Perna et al. } 2016 \\
\text { [108]: Bayesian } \\
\text { meta-analysis of } \\
\text { hazelnuts }\end{array}$ & $\begin{array}{l}\text { Gobbo et al. } 2015 \\
\text { [180]: per } 1 \\
\text { serving/day of } \\
\text { tree nuts vs. } \\
\text { controls }\end{array}$ & $\begin{array}{l}\text { Mohammadifard } \\
\text { et al. } 2015 \text { [181]: } \\
\text { nuts vs. controls }\end{array}$ & $\begin{array}{c}\text { Mejia et al. } 2014 \\
\text { [144]: } \sim 50 \mathrm{~g} / \text { day } \\
\text { of tree nuts over } \\
\sim 8 \text { weeks vs. } \\
\text { controls }\end{array}$ & $\begin{array}{l}\text { Viguiliouk et al. } \\
2014 \text { [3]: tree } \\
\text { nuts vs. controls } \\
\text { in subjects with } \\
\text { T2DM }\end{array}$ & $\begin{array}{c}\text { Sabate et al } \\
\text { 2010 [182]: } 67 \\
\text { g/day of nuts } \\
\text { vs. controls }\end{array}$ & $\begin{array}{l}\text { Phung et al. } \\
\text { 2009 [183]: } \\
\text { almonds } \\
\text { (25-169 g/day) } \\
\text { vs. controls } \\
\end{array}$ & $\begin{array}{c}\text { Banel et al. } \\
2009 \text { [112]: } \\
\text { walnuts vs. } \\
\text { controls }\end{array}$ \\
\hline LDL-C & $\begin{array}{c}-5.51 \mathrm{mg} / \mathrm{dL} \\
(95 \% \mathrm{CI}-7.72, \\
-3.29 \mathrm{mg} / \mathrm{dL} ; \\
p \text { overall effect } \\
<0.001 ; \mathrm{I}^{2}= \\
0.0 \% ; p \text {-het }= \\
0.49) \\
=-0.14 \\
\mathrm{mmol} / \mathrm{L}(95 \% \\
\mathrm{CI}-0.2, \\
-0.085 \\
\mathrm{mmol} / \mathrm{L})\end{array}$ & $\begin{array}{c}-0.69 \mathrm{mg} / \mathrm{dL} \\
(95 \% \mathrm{CI}-1.32, \\
-0.07 ; p \\
\text { overall effect }< \\
0.05) \\
=-0.017 \\
\mathrm{mmol} / \mathrm{L}(95 \% \\
\mathrm{CI}-0.03, \\
-0.002 \\
\mathrm{mmol} / \mathrm{L})\end{array}$ & $\begin{array}{c}-0.124 \mathrm{mmol} / \mathrm{L} \\
(95 \% \mathrm{CI}-0.196, \\
-0.051 \mathrm{mmol} / \mathrm{L} ; \\
p \text { overall effect = } \\
0.001)\end{array}$ & $\begin{array}{c}\mathrm{MD} \Delta=-0.150 \\
\mathrm{mmol} / \mathrm{L}(95 \% \\
\mathrm{HPD}-0.308 \\
-0.003)\end{array}$ & $\begin{array}{c}-4.8 \mathrm{mg} / \mathrm{dL} \\
(95 \% \mathrm{CI}-5.5 \\
-4.2 \mathrm{mg} / \mathrm{dL} ; \mathrm{I}^{2}> \\
30 \% ; p-\text { het }=0.01 ; \\
p \text { overall effect }< \\
0.05) \\
=-0.12 \mathrm{mmol} / \mathrm{L} \\
(95 \% \mathrm{CI}-0.14 \\
-0.11 \mathrm{mmol} / \mathrm{L})\end{array}$ & & & & $\begin{array}{c}-10.2 \mathrm{mg} / \mathrm{dL} \\
(95 \% \mathrm{CI}-13.1, \\
-7.4 \mathrm{mg} / \mathrm{dL} ; \\
7.4 \% \text { change; } p \\
\text { overall effect }< \\
0.001) \\
=-0.26 \\
\mathrm{mmol} / \mathrm{L}(95 \% \\
\mathrm{CI}-0.34 \\
-0.19 \\
\mathrm{mmol} / \mathrm{L})\end{array}$ & $\begin{array}{c}-0.15 \mathrm{mmol} / \mathrm{L} \\
(95 \% \mathrm{CI}-0.29, \\
0.0001 \\
\mathrm{mmol} / \mathrm{L} ; \mathrm{I}^{2}= \\
0.0 \% ; p \text {-het }= \\
\mathrm{NS} ; p \text { overall } \\
\text { effect }=0.05)\end{array}$ & $\begin{array}{c}-9.2 \mathrm{mg} / \mathrm{dL} \\
(95 \% \mathrm{CI}-13.1 \\
-5.36 \mathrm{mg} / \mathrm{dL} ; \\
\mathrm{I}^{2}=0.0 \% ; p \text {-het } \\
=0.65 ; p \\
\text { overall effect } \\
0.001) \\
=-0.24 \\
\mathrm{mmol} / \mathrm{L}(95 \% \\
\mathrm{CI}-0.34 \\
-0.138 \\
\mathrm{mmol} / \mathrm{L})\end{array}$ \\
\hline HDL-C & $\begin{array}{c}0.10 \mathrm{mg} / \mathrm{dL} \\
(95 \% \mathrm{CI}-0.78, \\
0.97 \mathrm{mg} / \mathrm{dL} ; p \\
\text { overall effect = } \\
0.83 ; \mathrm{I}^{2}=0.0 \% \text {; } \\
p \text {-het }=0.85) \\
=0.003 \\
\mathrm{mmol} / \mathrm{L}(95 \% \\
\mathrm{CI}-0.02,0.025 \\
\mathrm{mmol} / \mathrm{L})\end{array}$ & $\begin{array}{c}0.54 \mathrm{mg} / \mathrm{dL} \\
(95 \% \mathrm{CI} 0.17, \\
0.90 ; p \text { overall } \\
\text { effect }<0.05) \\
0.014 \mathrm{mmol} / \mathrm{L} \\
(95 \% \mathrm{CI} 0.004, \\
0.023 \mathrm{mmol} / \mathrm{L})\end{array}$ & $\begin{array}{c}-0.017 \mathrm{mmol} / \mathrm{L} \\
(95 \% \mathrm{CI}-0.043 \\
\mathrm{mmol} / \mathrm{L}, 0.009 ; p \\
\text { overall effect }= \\
0.207)\end{array}$ & $\begin{array}{c}\mathrm{MD} \Delta=0.002 \\
\mathrm{mmol} / \mathrm{L}(95 \% \\
\mathrm{HPD}-0.140, \\
0.147 \mathrm{mmol} / \mathrm{L})\end{array}$ & $\begin{array}{c}-0.3 \mathrm{mg} / \mathrm{dL} \\
(95 \% \mathrm{CI}-0.9,0.4 ; \\
\mathrm{I}^{2}>30 \% ; p \text {-het }= \\
0.33 ; p \text { overall } \\
\text { effect = NS) } \\
-0.007 \mathrm{mmol} / \mathrm{L} \\
(95 \% \mathrm{CI}-0.02 \\
0.01 \mathrm{mmol} / \mathrm{L})\end{array}$ & & $\begin{array}{c}0.00 \mathrm{mmol} / \mathrm{L} \\
(95 \% \mathrm{CI}-0.01 \\
0.01 \mathrm{mmol} / \mathrm{L} ; \mathrm{I}^{2} \\
=86 \% ; p \text {-het }< \\
0.00001 ; p \text { overall } \\
\text { effect }=0.93) .\end{array}$ & & $\begin{array}{c}0.09 \mathrm{mg} / \mathrm{dL} \\
(95 \% \mathrm{CI}-1.00 \\
1.19 \mathrm{mg} / \mathrm{dL} ; p \\
\text { overall effect }= \\
\mathrm{NS}) \\
=0.002 \\
\mathrm{mmol} / \mathrm{L}(95 \% \\
\mathrm{CI}-0.026,0.03 \\
\mathrm{mmol} / \mathrm{L})\end{array}$ & $\begin{array}{l}-0.05 \mathrm{mmol} / \mathrm{L} \\
(95 \% \mathrm{CI}-0.10, \\
0.01 \mathrm{mmol} / \mathrm{L} ; \\
\mathrm{I}^{2}=0.0 \% ; p \text {-het } \\
=\mathrm{NS} ; p \text { overall } \\
\text { effect }=0.08)\end{array}$ & $\begin{array}{c}-0.2 \mathrm{mg} / \mathrm{dL} \\
(95 \% \mathrm{CI}-1.79 \\
1.38 \mathrm{mg} / \mathrm{dL} ; \mathrm{I}^{2} \\
=0.0 \% ; p \text {-het }= \\
0.8 ; p \text { overall } \\
\text { effect }=0.8) \\
=-0.005 \\
\mathrm{mmol} / \mathrm{L}(95 \% \\
\mathrm{CI}-0.05,0.036 \\
\mathrm{mmol} / \mathrm{L}) \\
\end{array}$ \\
\hline TG & $\begin{array}{c}-4.69 \mathrm{mg} / \mathrm{dL} \\
(95 \% \mathrm{CI}-8.93, \\
-0.45 \mathrm{mg} / \mathrm{dL} ; \\
p \text { overall effect } \\
=0.03 ; \mathrm{I}^{2}= \\
0.0 \% ; p \text {-het }= \\
0.99) \\
=-0.052 \\
\mathrm{mmol} / \mathrm{L}(95 \% \\
\mathrm{CI}-0.1 \\
-0.005 \\
\mathrm{mmol} / \mathrm{L})\end{array}$ & $\begin{array}{c}-0.66 \mathrm{mg} / \mathrm{dL} \\
(95 \% \mathrm{CI}-1.34, \\
0.01 ; p \text { overall } \\
\text { effect }=\mathrm{NS}) \\
=-0.007 \\
\mathrm{mmol} / \mathrm{L}(95 \% \\
\mathrm{CI}-0.015,0.00 \\
\mathrm{mmol} / \mathrm{L})\end{array}$ & $\begin{array}{c}-0.067 \mathrm{mmol} / \mathrm{L} \\
(95 \% \mathrm{CI}-0.132, \\
-0.002 \mathrm{mmol} / \mathrm{L} ; \\
p \text { overall effect }= \\
0.042)\end{array}$ & $\begin{array}{c}\mathrm{MD} \Delta=0.045 \\
\mathrm{mmol} / \mathrm{L}(95 \% \\
\mathrm{HPD}-0.195 \\
0.269)\end{array}$ & $\begin{array}{c}-2.2 \mathrm{mg} / \mathrm{dL} \\
(95 \% \mathrm{CI}-3.8 \\
-0.5 \mathrm{mg} / \mathrm{dL} ; \mathrm{I}^{2}> \\
30 \% ; p \text {-het }=0.16 ; \\
p \text { overall effect }< \\
0.05) \\
=0.02 \mathrm{mmol} / \mathrm{L} \\
(95 \% \mathrm{CI}-0.04 \\
-0.006 \mathrm{mmol} / \mathrm{L})\end{array}$ & & $\begin{array}{c}-0.06 \mathrm{mmol} / \mathrm{L} \\
(95 \% \mathrm{CI}-0.09 \\
-0.03 ; \mathrm{I}^{2}=34 \% \\
p \text {-het }=0.02 ; p \\
\text { overall effect }< \\
0.0001)\end{array}$ & & $\begin{array}{c}-20.6 \mathrm{mg} / \mathrm{dL} \\
(10.2 \% \text { change; } \\
(95 \% \mathrm{CI}-30.7, \\
-9.9 \mathrm{mg} / \mathrm{dL} ; p \\
\text { overall effect }< \\
0.05) \text { in } \\
\text { subjects with } \\
\text { TG of } 150 \\
\mathrm{mg} / \mathrm{dL} . \\
=-0.23 \\
\mathrm{mmol} / \mathrm{L}(95 \% \\
\mathrm{CI}-0.3,-0.1 \\
\mathrm{mmol} / \mathrm{L})\end{array}$ & $\begin{array}{l}-0.04 \mathrm{mmol} / \mathrm{L} \\
(95 \% \mathrm{CI}-0.20, \\
0.11 \mathrm{mmol} / \mathrm{L} ; \\
\mathrm{I}^{2}=0.0 \% ; p \text {-het } \\
=\mathrm{NS} ; p \text { overall } \\
\text { effect }=0.58)\end{array}$ & $\begin{array}{c}-3.9 \mathrm{mg} / \mathrm{dL} \\
(95 \% \mathrm{CI} \\
-11.92,4.20 \\
\mathrm{mg} / \mathrm{dL} ; \mathrm{I}^{2} \\
=0.0 \% ; p \text {-het }= \\
0.99 ; p \text { overall } \\
\text { effect }=0.3) \\
=-0.04 \\
\mathrm{mmol} / \mathrm{L}(95 \% \\
\mathrm{CI}-0.13,0.05 \\
\mathrm{mmol} / \mathrm{L})\end{array}$ \\
\hline
\end{tabular}


Table 4. Cont.

\begin{tabular}{|c|c|c|c|c|c|c|c|c|c|c|c|}
\hline \multicolumn{12}{|c|}{ References Comparison } \\
\hline Variables & $\begin{array}{l}\text { Guasch-Ferre } \\
\text { et al. } 2018 \text { [94]: } \\
\text { walnuts vs. } \\
\text { controls }\end{array}$ & $\begin{array}{l}\text { Mazidi et al. } \\
2016 \text { [178]: } \\
\text { nuts vs. } \\
\text { controls }\end{array}$ & $\begin{array}{l}\text { Musa-Veloso et } \\
\text { al. 2016 [179]: } \\
\text { almonds vs. } \\
\text { controls }\end{array}$ & $\begin{array}{l}\text { Perna et al. } 2016 \\
\text { [108]: Bayesian } \\
\text { meta-analysis of } \\
\text { hazelnuts }\end{array}$ & $\begin{array}{l}\text { Gobbo et al. } 2015 \\
\text { [180]: per } 1 \\
\text { serving/day of } \\
\text { tree nuts vs. } \\
\text { controls }\end{array}$ & $\begin{array}{l}\text { Mohammadifard } \\
\text { et al. } 2015 \text { [181]: } \\
\text { nuts vs. controls }\end{array}$ & $\begin{array}{c}\text { Mejia et al. } 2014 \\
\text { [144]: } \sim 50 \mathrm{~g} / \text { day } \\
\text { of tree nuts over } \\
\sim 8 \text { weeks vs. } \\
\text { controls }\end{array}$ & $\begin{array}{l}\text { Viguiliouk et al. } \\
2014 \text { [3]: tree } \\
\text { nuts vs. controls } \\
\text { in subjects with } \\
\text { T2DM }\end{array}$ & $\begin{array}{l}\text { Sabate et al } \\
\text { 2010 [182]: } 67 \\
\text { g/day of nuts } \\
\text { vs. controls }\end{array}$ & $\begin{array}{l}\text { Phung et al. } \\
2009 \text { [183]: } \\
\text { almonds } \\
\text { (25-169 g/day) } \\
\text { vs. controls }\end{array}$ & $\begin{array}{c}\text { Banel et al. } \\
2009 \text { [112]: } \\
\text { walnuts vs. } \\
\text { controls }\end{array}$ \\
\hline ApoA1 & $\begin{array}{c}-2.91 \mathrm{mg} / \mathrm{dL} \\
(95 \% \mathrm{CI}-5.98, \\
0.08 \mathrm{mg} / \mathrm{dL} ; p \\
\text { overall effect }= \\
0.057 ; \mathrm{I}^{2}= \\
0.0 \% ; p \text {-het }= \\
0.822)\end{array}$ & $\begin{array}{c}1.38 \mathrm{mg} / \mathrm{dL} \\
(95 \% \mathrm{CI} 0.15 \\
2.61 \mathrm{mg} / \mathrm{dL} ; p \\
\text { overall effect }< \\
0.05)\end{array}$ & & & $\begin{array}{c}-0.6 \mathrm{mg} / \mathrm{dL} \\
(95 \% \mathrm{CI}-1.9,0.7 \\
\mathrm{mg} / \mathrm{dL} ; \mathrm{I}^{2}>30 \% ; \\
p \text {-het }=0.38 ; p \\
\text { overall effect }= \\
\text { NS) }\end{array}$ & & & & & & \\
\hline TC: HDL-C & & & $\begin{array}{c}-0.207(95 \% \text { CI } \\
-0 \cdot 362,-0.052 ; \\
p \text { overall effect }= \\
0.009)\end{array}$ & & & & & & $\begin{array}{c}-0.24(5.6 \% \\
\text { change; } p \\
\text { overall effect }= \\
0.001)\end{array}$ & & \\
\hline $\begin{array}{l}\text { LDL-C: } \\
\text { HDL-C }\end{array}$ & & & $\begin{array}{c}-0.089(95 \% \text { CI } \\
-0.209,0.031 ; p \\
\text { overall effect }= \\
0.145)\end{array}$ & & & & & & $\begin{array}{c}-0.22(8.3 \% \\
\text { change; } p \\
\text { overall effect }= \\
0.001)\end{array}$ & $\begin{array}{c}-0.04(95 \% \text { CI } \\
-0.21,0.14 ; I^{2} \\
=0.0 \% ; p \text {-het }= \\
\text { NS; } p \text { overall } \\
\text { effect }=0.670)\end{array}$ & \\
\hline ApoB & $\begin{array}{c}-3.74 \mathrm{mg} / \mathrm{dL} ; \\
95 \% \mathrm{CI}-6.51 ; \\
-0.97 \mathrm{mg} / \mathrm{dL} ; \\
p \text { overall effect } \\
=0.008 ; \mathrm{I}^{2}= \\
0.0 \% ; p \text {-het }= \\
0.793)\end{array}$ & & & & $\begin{array}{c}-3.7 \mathrm{mg} / \mathrm{dL} \\
(95 \% \mathrm{CI}-5.2 \\
-2.3 \mathrm{mg} / \mathrm{dL} ; \mathrm{I}^{2}> \\
30 \% ; p \text {-het }=0.17 ; \\
p \text { overall effect }< \\
0.05)\end{array}$ & & & & & & \\
\hline ApoB100 & & $\begin{array}{c}-1.50 \mathrm{mg} / \mathrm{dL} \\
(95 \% \mathrm{CI}-2.43, \\
0.57 ; p \text { overall } \\
\text { effect }=\mathrm{NS})\end{array}$ & & & $\begin{array}{c}-2.8 \mathrm{mg} / \mathrm{dL} \\
(95 \% \mathrm{CI}-6.2,0.7 \\
\mathrm{mg} / \mathrm{dL} ; \mathrm{I}^{2}>30 \% ; \\
p \text {-het }=0.31 ; p \\
\text { overall effect }= \\
\text { NS) }\end{array}$ & & & & & & \\
\hline
\end{tabular}


Table 4. Cont.

\begin{tabular}{|c|c|c|c|c|c|c|c|c|c|c|c|}
\hline \multicolumn{12}{|c|}{ References Comparison } \\
\hline Variables & $\begin{array}{l}\text { Guasch-Ferre } \\
\text { et al. } 2018 \text { [94]: } \\
\text { walnuts vs. } \\
\text { controls }\end{array}$ & $\begin{array}{l}\text { Mazidi et al. } \\
\text { 2016 [178]: } \\
\text { nuts vs. } \\
\text { controls }\end{array}$ & $\begin{array}{l}\text { Musa-Veloso et } \\
\text { al. 2016 [179]: } \\
\text { almonds vs. } \\
\text { controls }\end{array}$ & $\begin{array}{l}\text { Perna et al. } 2016 \\
\text { [108]: Bayesian } \\
\text { meta-analysis of } \\
\text { hazelnuts }\end{array}$ & $\begin{array}{l}\text { Gobbo et al. } 2015 \\
\text { [180]: per } 1 \\
\text { serving/day of } \\
\text { tree nuts vs. } \\
\text { controls } \\
\end{array}$ & $\begin{array}{l}\text { Mohammadifard } \\
\text { et al. } 2015 \text { [181]: } \\
\text { nuts vs. controls }\end{array}$ & $\begin{array}{l}\text { Mejia et al. } 2014 \\
\text { [144]: } \sim 50 \mathrm{~g} / \text { day } \\
\text { of tree nuts over } \\
\sim 8 \text { weeks vs. } \\
\text { controls }\end{array}$ & $\begin{array}{l}\text { Viguiliouk et al. } \\
2014 \text { [3]: tree } \\
\text { nuts vs. controls } \\
\text { in subjects with } \\
\text { T2DM }\end{array}$ & $\begin{array}{l}\text { Sabate et al } \\
2010 \text { [182]: } 67 \\
\text { g/day of nuts } \\
\text { vs. controls }\end{array}$ & $\begin{array}{l}\text { Phung et al. } \\
2009 \text { [183]: } \\
\text { almonds } \\
\text { (25-169 g/day) } \\
\text { vs. controls } \\
\end{array}$ & $\begin{array}{l}\text { Banel et al. } \\
2009 \text { [112]: } \\
\text { walnuts vs. } \\
\text { controls }\end{array}$ \\
\hline SBP & $\begin{array}{c}-0.72 \mathrm{mmHg} \\
(95 \% \mathrm{CI}-2.75, \\
1.30 \mathrm{mmHg} ; p \\
\text { overall effect }= \\
\text { NS) }\end{array}$ & $\begin{array}{c}-0.69 \mathrm{mmHg} \\
(95 \% \mathrm{CI}-1.34 \\
\mathrm{mmHg}, 0.03 ; p \\
\text { overall effect = } \\
\mathrm{NS})\end{array}$ & & & $\begin{array}{c}0.3 \mathrm{mmHg}(95 \% \\
\mathrm{CI}-0.8,1.4 \\
\mathrm{mmHg} ; \mathrm{I}^{2}>30 \% ; \\
p \text {-het }=0.001 ; p \\
\text { overall effect = } \\
\text { NS) }\end{array}$ & $\begin{array}{c}-0.91 \mathrm{mmHg}(95 \% \\
\text { CI }-2.18,0.36 \\
\text { mmHg; } \mathrm{I}^{2}=73.8 \% \\
p \text {-het }<0.001 ; p \\
\text { overall effect }=\mathrm{NS}) \\
\text { for all type nuts. } \\
-1.29 \mathrm{mmH} \\
\left(-2.35,-0.22 ; \mathrm{I}^{2}=\right. \\
53.3 \% ; p \text {-het }= \\
0.002 ; p \text { overall } \\
\text { effect }=0.02) \text { for all } \\
\text { type nuts } \# .-1.82 \\
\text { mmHg }(-2.97 \\
-0.67 ; p \text {-het: } \mathrm{NS} \\
p \text { overall effect }= \\
0.002) \text { for } \\
\text { pistachios }\end{array}$ & $\begin{array}{c}0.07 \mathrm{mmHg}(95 \% \\
\mathrm{CI}-1.54,1.69 \\
\mathrm{mmHg} ; \mathrm{I}^{2}=64 \% \text {; } \\
p \text {-het }<0.0001 ; p \\
\text { overall effect = } \\
\text { NS) }\end{array}$ & & & & \\
\hline DBP & $\begin{array}{c}0.10 \mathrm{mmHg} \\
(95 \% \mathrm{CI}-1.49, \\
1.30 \mathrm{mmHg} ; p \\
\text { overall effect= } \\
\text { NS) }\end{array}$ & $\begin{array}{c}-0.14 \mathrm{mmHg} \\
(95 \% \mathrm{CI}-0.54, \\
0.25 \mathrm{mmHg} ; p \\
\text { overall effect = } \\
\text { NS) }\end{array}$ & & & $\begin{array}{c}0.4 \mathrm{mmHg}(95 \% \\
\mathrm{CI}-0.8,1.6 \\
\mathrm{mmHg} ; \mathrm{I}^{2}>30 \% \text {; } \\
p \text {-het }=0.32 ; p \\
\text { overall effect }= \\
\text { NS) }\end{array}$ & $\begin{array}{c}0.21 \mathrm{mmHg}(95 \% \\
\text { CI }-0.54,0.97 \\
\text { mmHg; } \mathrm{I}^{2}=69.6 \% ; \\
p \text {-het }<0.001 ; p \\
\text { overall effect }=\mathrm{NS}) \\
\text { for all type nuts. } \\
-1.19 \text { mmHg }(95 \% \\
\mathrm{CI}-2.35,-0.03 ; p \\
\text { overall effect }= \\
0.04 ; p \text {-het }=\mathrm{NS}) \\
\text { for mixed nuts. } \\
-0.80 \mathrm{mmHg}(95 \% \\
\mathrm{CI}-1.43,-0.17 ; \\
p \text {-het }=0.44 ; \mathrm{I}^{2}= \\
0.0 \% ; p \text { overall } \\
\text { effect }=0.04) \text { for } \\
\text { pistachios } \#\end{array}$ & $\begin{array}{c}0.23 \mathrm{mmHg}(95 \% \\
\mathrm{CI}-0.38,0.83 \\
\mathrm{mmHg} ; \mathrm{I}^{2}=34 \% \text {; } \\
p \text {-het }=0.07 ; p \\
\text { overall effect = } \\
\text { NS) }\end{array}$ & & & & \\
\hline
\end{tabular}

\#: only in subjects without T2DM. ApoA1, apolipoprotein A1; ApoB, apolipoprotein B; ApoB100, apolipoprotein B100; CRP, C-reactive protein; DBP, diastolic blood pressure; FBG, fasting blood glucose; HOMA-IR, homeostasis model assessment of insulin resistance index; het, heterogeneity; HDL-C, high density lipoprotein cholesterol; $95 \%$ HPD, 95\% highest posterior from baseline across treatment; NS, non-significant; RCT, randomized controlled trial; SBP, systolic blood pressure; TC, total cholesterol; TG, triglyceride; T2DM, type 2 diabetes mellitus; TNF- $\alpha$, tumor necrosis factor-alpha; VCAM-1, vascular cell adhesion molecule-1. 
Table 5. Summary of nut consumption and adipokines and inflammatory markers in intervention studies.

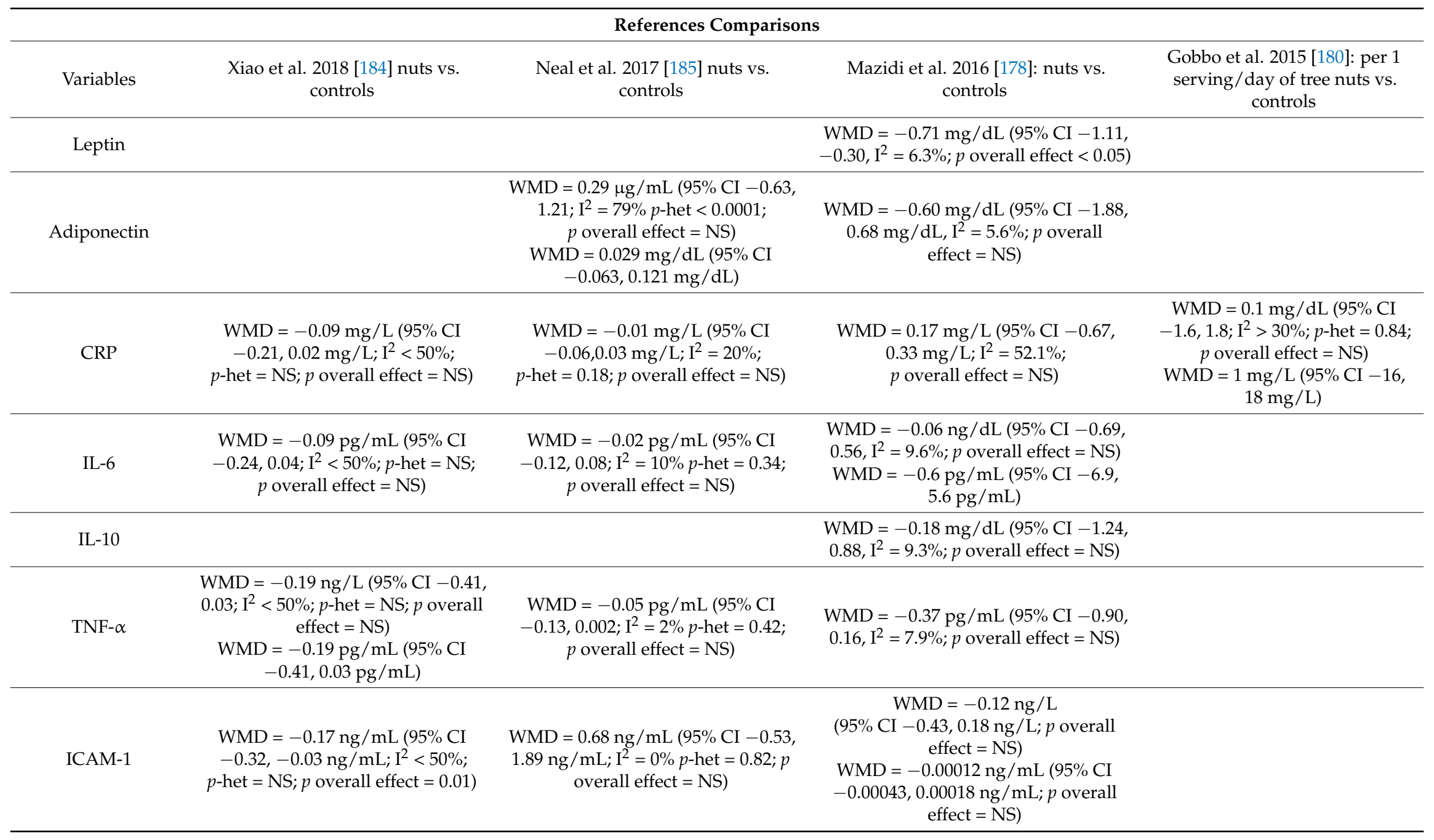


Table 5. Cont.

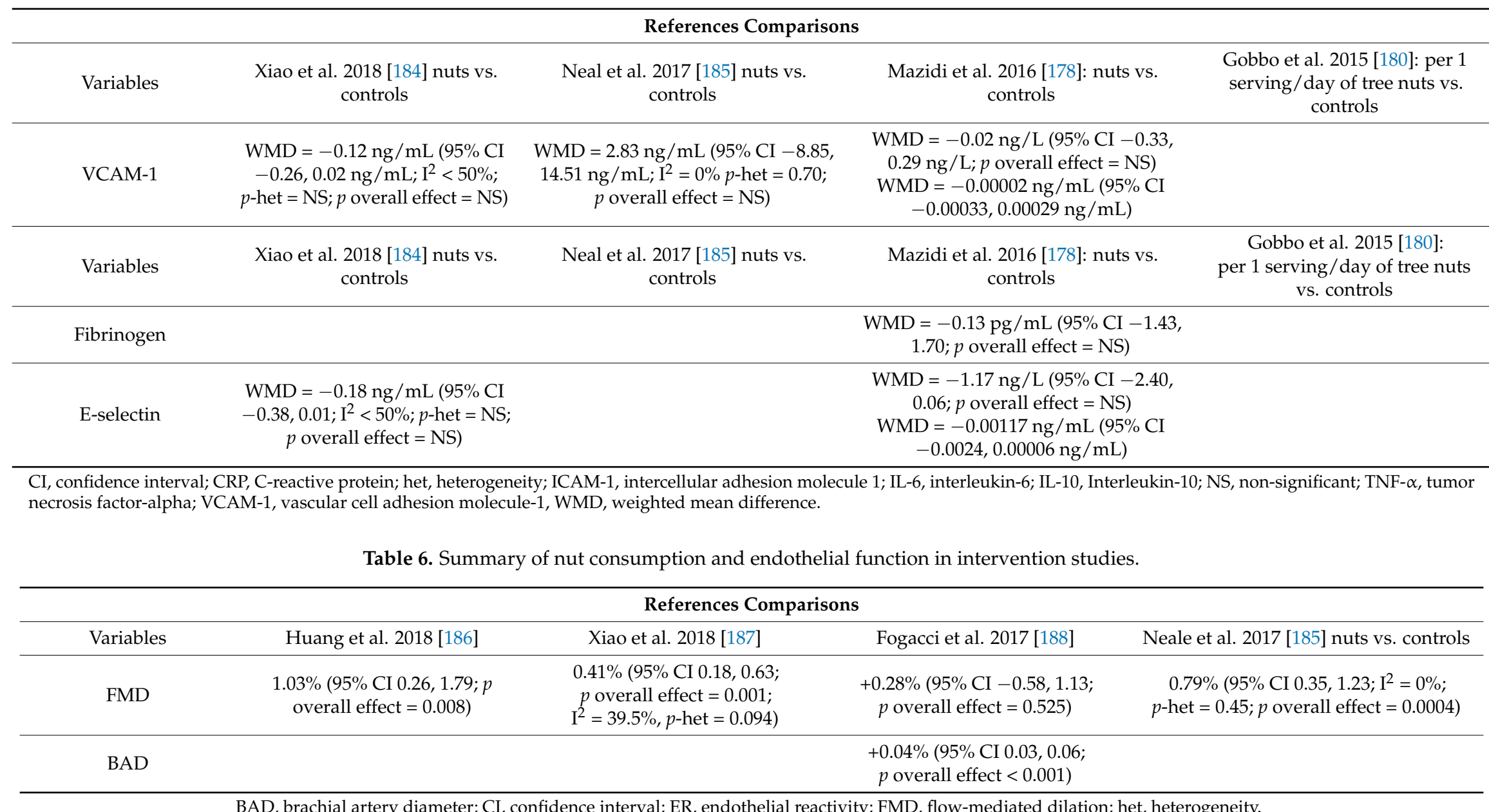

BAD, brachial artery diameter; CI, confidence interval; ER, endothelial reactivity; FMD, flow-mediated dilation; het, heterogeneity. 


\subsubsection{Glycemic Control}

Three meta-analyses $[3,144,178]$ of intervention studies have found nuts lowered fasting glucose levels with a reduction ranging from 0.08 to $0.15 \mathrm{mmol} / \mathrm{L}$ compared with control diets. In three meta-analyses, control diets were a low-fat diet, a high-fat diet, a libitum diet without nuts, a refined carbohydrate snack, a diet without nuts and a usual diet. The type of nuts were almonds, walnuts, cashews, hazelnuts, cashew and pistachios. The study durations ranged from 4 weeks to 12 months.

A meta-analysis of 20 RCTs [100,103,110,122,132,147,151,172-174,177,189-197] was conducted by Mazidi et al. 2016 [178]. Nut consumption significantly reduced fasting blood glucose to a small degree $(\mathrm{MD}=-1.45 \mathrm{mg} / \mathrm{dL}(0.08 \mathrm{mmol} / \mathrm{L}) ; 95 \% \mathrm{CI}-2.20,0.70 ; p<0.05)$.

Mejia et al. 2014 [144] conducted a meta-analysis of 49 RCTs with $\geq 3$-week durations ( $68.8 \%$ of studies were $<12$-week durations) in 2226 subjects with at least one characteristic of the metabolic syndrome A fasting glucose analysis (26 studies; $n=1360$ ) was conducted with otherwise healthy subjects $[133,196]$, subjects with dyslipidemia [198,199], metabolic syndrome $[99,105,107,132,137,145-$ $148,171,200]$ and T2DM [95,100,125,143,149,150,175,201-203]. Tree nut consumption at median dose of $\sim 50 \mathrm{~g} /$ day for a median of 8 weeks significantly reduced fasting blood glucose (Mean difference $(\mathrm{MD})=-0.08 \mathrm{mmol} / \mathrm{L}(95 \% \mathrm{CI}-0.16,-0.01 \mathrm{mmol} / \mathrm{L}))$ compared with control diets. Although statistically significant this is a trivial change clinically.

Viguiliouk et al. 2014 [3] focused on subjects with T2DM in a meta-analysis of 12 RCTs ( $n=450)$ of $\geq 3$ weeks duration to investigate the effects of tree nut consumption on glycemic control (glycosylated hemoglobin ( $\mathrm{HbA} 1 \mathrm{c})$, fasting glucose, fasting insulin and homeostasis model assessment of insulin resistance index (HOMA-IR)) compared with isocaloric diets without tree nuts. This meta-analysis showed that tree nut consumption at a median dose of $56 \mathrm{~g} /$ day over a median follow-up period of $\sim 8$ weeks significantly decreased $\mathrm{HbA1c}(\mathrm{MD}=-0.07 \% ; p=0.0003$ ) and fasting glucose (MD $=-0.15 \mathrm{mmol} / \mathrm{L} ; p=0.03$ ) compared with control diets. However, fasting insulin and HOMA-IR were not altered. Most RCTs analyzed in this meta-analysis had short study periods and poor study quality [3].

\subsubsection{Blood Lipids}

In a recent meta-analysis of RCTs by Guasch-Ferre et al. 2018 [94], a diet rich in walnuts significantly reduced total cholesterol $(\mathrm{TC})(\mathrm{WMD}=-6.99 \mathrm{mg} / \mathrm{dL} ; 95 \% \mathrm{CI}-9.39,-4.58 \mathrm{mg} / \mathrm{dL}$; $p<0.001 ; \mathrm{I}^{2}=0.0 \% ; p$-het $\left.=0.64\right)$, low density lipoprotein cholesterol (LDL-C) $(\mathrm{WMD}=-5.51 \mathrm{mg} / \mathrm{dL}$; $95 \% \mathrm{CI}-7.72,-3.29 \mathrm{mg} / \mathrm{dL} ; p<0.001 ; \mathrm{I}^{2}=0.0 \% ; p$-het $\left.=0.49\right)$ and TG $(\mathrm{WMD}=-4.69 ; 95 \% \mathrm{CI}$ $-8.93,-0.45 ; p=0.03 ; \mathrm{I}^{2}=0.0 \%$; $p$-het $\left.=0.99\right)$ by $3.25 \%, 3.73 \%$ and $5.52 \%$ respectively compared with control diets. HDL-C was not altered by a diet rich in walnuts compared with control diets $\left(\mathrm{WMD}=0.10 \mathrm{mg} / \mathrm{dL} ; 95 \% \mathrm{CI}-0.78,0.97 \mathrm{mg} / \mathrm{dL} ; p=0.83 ; \mathrm{I}^{2}=0.0 \% ; p\right.$-het $\left.=0.85\right)$. Apolipoprotein $\mathrm{B}$ $\left(\right.$ ApoB) was reduced $\left(\mathrm{WMD}=-3.74 \mathrm{mg} / \mathrm{dL} ; 95 \% \mathrm{CI}-6.51,-0.97 ; p=0.008 ; \mathrm{I}^{2}=0.0 \% ; p\right.$-het $\left.=0.793\right)$ with no significant change in ApoA1 (WMD $=-2.91 \mathrm{mg} / \mathrm{dL} ; 95 \% \mathrm{CI}-5.98,0.08 ; p=0.057 ; \mathrm{I}^{2}=0.0 \%$; $p$-het $=0.822$ ) compared with control diets [94].

In this meta-analysis, 24 studies from 23 publications [95-101,103-105,115-119,196,204-210] including 1020 subjects for TC and LDL-C were analyzed. Twenty-five studies from 24 publications [95107,115-119,196,204,206-208,210] including 1059 subjects for HDL-C were analyzed. Twenty-four studies from 23 publications [95-100,102-105,107,115-119,196,204,206-210] including 1059 subjects for TG were analyzed. Nine studies from 8 publications (370 subjects) [97-99,103,104,115,119,205] for ApoA1 and 11 studies from 10 publications (604 subjects) [97-99,103,104,115,119,196,204,205] for ApoB were analyzed.

Similar results were found by Banel et al. 2009 [112] who conducted a meta-analysis of 13 RCTs $[96,97,101-104,113-119]$ with a focus on the effects of walnuts (10-24\% of total calories; for 4-24 weeks (mean $=\sim 6$ weeks) on lipid profiles in comparison with control diets. Walnut consumption significantly reduced TC (MD $=-10.3 \mathrm{mg} / \mathrm{dL} ; p<0.001)$ and LDL-C $(\mathrm{MD}=-9.2 \mathrm{mg} / \mathrm{dL}$; $p<0.001)$ compared with control diets in 11 studies (11 publications) $[96,97,101,103,104,113,115-119]$. 
HDL-C (MD $=-0.2 \mathrm{mg} / \mathrm{dL} ; p=0.8)$ and triglyceride $(\mathrm{TG})(\mathrm{MD}=-3.9 \mathrm{mg} / \mathrm{dL} ; p=0.3)$ were not altered compared with control diets.

A meta-analysis of 27 studies (17 parallel and 10 crossover studies) from 18 publications [98,122, $125,133,134,137,139,143,146,156,159,162,202,203,211-214]$, which was conducted by Musa-Veloso et al. 2016 [179] showed a significantly decrease in fasting, TC, LDL-C and TG but no alteration in HDL-C; $-0.017 \mathrm{mmol} / \mathrm{L} ; p=0.207$ ), following almond consumption (20-113 $\mathrm{g} /$ day) in periods ranging from 4 weeks to 18 months. With regard to TC levels, $73 \%$ of all 27 studies were $<12$ weeks long and $63 \%$ used $\geq 45 \mathrm{~g}$ of almond/day. TC levels from all 27 strata was decreased by $-0.153 \mathrm{mmol} / \mathrm{L}(95 \% \mathrm{CI}$ $-0.235,-0.070 \mathrm{mmol} / \mathrm{L} ; p<0.001)$. LDL-C in 25 studies from 17 publications $[98,122,125,133,134,137$, $139,143,146,156,159,162,202,203,211,212,214]$ was reduced by $-0.124 \mathrm{mmol} / \mathrm{L}(95 \% \mathrm{CI}-0.196,-0.051$ $\mathrm{mmol} / \mathrm{L} ; p=0.001)$. TG levels from 25 studies from 17 publications $[98,122,125,133,137,139,143,146$, $156,159,162,202,203,211-214]$ were decreased by $-0.067 \mathrm{mmol} / \mathrm{L}(95 \% \mathrm{CI}-0.132,-0.002 \mathrm{mmol} / \mathrm{L}$; $p=0.042)$.

A meta-analysis of 20 RCTs [100,103,110,122,132,147,151,172-174,177,189-197] conducted by Mazidi et al. 2016 [178] showed that nut consumption significantly reduced TC (MD $=-0.82 \mathrm{mg} / \mathrm{dL})$, LDL-C (MD $=-0.69 \mathrm{mg} / \mathrm{dL}), \mathrm{HDL}-\mathrm{C}(\mathrm{MD}=0.54 \mathrm{mg} / \mathrm{dL})$ and ApoAI $(\mathrm{MD}=1.38 \mathrm{mg} / \mathrm{dL})$.

Perna et al. 2016 [108] investigated the effects of hazelnut consumption (29-69 g/day; average $45 \mathrm{~g} /$ day) for 28-84 days (average 56 days) on lipid profiles using a Bayesian random effect meta-analysis. Hazelnuts in 3 studies [109-111] significantly decreased LDL-C (pooled MD $\Delta=-0.150 \mathrm{mmol} / \mathrm{L} ; 95 \%$ highest posterior density interval $(-0.308 ;-0.003)$.

Gobbo et al. 2015 [180] conducted a meta-analysis of 61 intervention trials (RCTs and non RCTs; $n=2582$ ) ranging from 3 to 26 weeks (median 4 weeks) to investigate the effects of tree nuts (walnuts $(n=21)$, almonds $(n=16)$, pistachios $(n=7)$, hazelnuts $(n=6)$, macadamia nuts $(n=4)$, pecans $(n=2)$, cashews $(n=2)$, mixed tree nuts $(n=2)$ and Brazil nuts $(n=1)]$ on TC, LDL-C, HDL-C and TG, Apo A1, ApoB and apo lipoprotein B100 in adults without CVD. An inverse-variance fixed-effects meta-analysis was used to calculate mean differences between nut intervention and control arms, dose-standardized to 1 serving $(28.4 \mathrm{~g}) / \mathrm{d}$. Nut consumption (per serving/d) significantly decreased TC $(-4.7 \mathrm{mg} / \mathrm{dL})$, LDL-C $(-4.8 \mathrm{mg} / \mathrm{dL})$, apolipoprotein B (ApoB; $-3.7 \mathrm{mg} / \mathrm{dL})$ and TG $(-2.2 \mathrm{mg} / \mathrm{dL})$. Tree nut consumption at $\geq 60 \mathrm{~g} /$ day showed a stronger effect on TC and LDL-C in a nonlinear manner ( $p$-nonlinearity < 0.001) and $100 \mathrm{~g}$ tree nuts/day decreased LDL-C by up to $35 \mathrm{mg} / \mathrm{dL}$. The ApoB reduction was greater in subjects with T2DM $(-11.5 \mathrm{mg} / \mathrm{dL} ; 95 \% \mathrm{CI}-16.2,-6.8 \mathrm{mg} / \mathrm{dL})$ than in nondiabetic subjects $(-2.5 \mathrm{mg} / \mathrm{dL} ; 95 \% \mathrm{CI}-4.7,-0.3 \mathrm{mg} / \mathrm{dL})(p$-het $=0.015)$.

Mejia et al. 2014 [144] conducted an analysis (44 studies; $n=1690$ ) with 42 publications including healthy subjects $[110,115,118,133,141,142,196,208,209,212,215,216]$, subjects with dyslipidemia $[104,119$, $122,127,129,134,214,217]$, metabolic syndrome $[102,105,110,132,137,139,145-148,171]$ and T2DM [95,96, $100,125,143,149,150,175,201-203]$. They found a significantly decrease in TG (MD $=-0.06 \mathrm{mmol} / \mathrm{L}$ $(95 \% \mathrm{CI}-0.09,-0.03 \mathrm{mmol} / \mathrm{L}))$ compared with a control diet. HDL-C was not altered after tree nuts consumption compared with a control diet [144].

In this review, the TC-lowering effect of nuts ranged from 0.021 to $0.28 \mathrm{mmol} / \mathrm{L}$ and LDL-C was lowered by 0.017 to $0.26 \mathrm{mmol} / \mathrm{L}$ comparing nuts to control diets. Interestingly, Banel et al. 2009 [112] found a bigger reduction in TC comparing walnuts with control diets. Part of the reason for the bigger reduction may be related to larger amount of walnuts consumed (30-108 g/day representing $5-25 \%$ of total energy).

\subsubsection{Adipokines and Inflammatory Markers}

A meta-analysis of 32 RCTs (36 publications) $[91,95,98,102,103,105,106,110,122,132,147,151,154$, $167,172-174,176,177,190,196,197,205,211,212,218-228])$ with $\geq 3$-week follow-up period conducted by Neale et al. 2017 [185], showed no significant alteration in adipokines/inflammatory markers including CRP $(\mathrm{WMD}=-0.01 \mathrm{mg} / \mathrm{L} ; 95 \% \mathrm{CI}-0.06,0.03 ; p=0.5926$ strata from 25 studies), adiponectin 
$(\mathrm{WMD}=0.29 \mu \mathrm{g} / \mathrm{mL} ; p=0.53), \mathrm{TNF}-\alpha(\mathrm{WMD}=-0.05 \mathrm{pg} / \mathrm{mL} ; p=0.17), \mathrm{IL}-6(\mathrm{WMD}=-0.02 \mathrm{pg} / \mathrm{mL}$; $p=0.65)$, ICAM-1 (WMD $=-0.68 \mathrm{ng} / \mathrm{mL} ; p=0.27)$ and VCAM-1 $(\mathrm{WMD}=2.83 \mathrm{ng} / \mathrm{mL} ; p=0.63)$.

In a very recent meta-analysis of RCT studies by Xiao et al. 2018 [184], nut consumption was shown to decrease intercellular adhesion molecule 1 (ICAM-1; WMD $=-0.17 ; 95 \% \mathrm{CI},-0.32,-0.03$; $p=0.01$ ) from 14 studies (13 publications) $[98,103,106,110,151,174,196,205,218,220,223,224,227]$.

A sub-analysis showed that this decreased effect of nuts was observed in the long-term studies ( $\geq 12$ weeks) and in parallel RCTs. No significant effects on C-reactive protein (CRP), tumor necrosis factor alpha (TNF- $\alpha$ ), vascular cell adhesion molecule (VCAM), IL-6, E-selectin were observed [184].

A meta-analysis of 20 RCTs (20 publications) [100,103,110,122,132,147,151,172-174,177,189-197] by Mazidi et al. 2016 [178] showed a significant effect of nuts on leptin (WMD $=-0.71 \mathrm{mg} / \mathrm{dL}$ ), with no effect on CRP, IL-6, adiponectin, IL-10 and TNF- $\alpha$ [178]. Biomarkers of endothelial function including E-selectin $(\mathrm{WMD}=-1.17 \mathrm{ng} / \mathrm{L})$, fibrinogen $(\mathrm{WMD}=-0.13 \mathrm{pg} / \mathrm{mL})$, ICAM-1 $(\mathrm{WMD}=-0.12 \mathrm{ng} / \mathrm{L})$ and VCAM-1 (WMD $=-0.02 \mathrm{ng} / \mathrm{L}$ ) were not significantly altered after nut consumption.

Gobbo et al. 2015 [180] also found that tree nut consumption per 1 serving (28.4 g)/day of tree nuts did not significantly alter CRP levels (WMD $=0.1 \mathrm{mg} / \mathrm{dL} ; 95 \% \mathrm{CI}-1.6,1.8$ ) compared with a control diet [180].

Overall it would appear that nuts have very little effects on inflammatory markers.

\subsubsection{Endothelial Function}

Large meta-analyses have found a beneficial effect of nut consumption on endothelial function.

Neale et al. 2017 [185] showed a significant improvement in flow-mediated dilation (FMD) (MD $=0.79 \% ; 95 \%$ CI 0.35, 1.23; $p=0.0004$ ) from 9 studies (8 publications $[95,103,105,173,174,176,219,228]$; 652 subjects). According to another meta-analysis which was conducted by Xiao et al. 2018 [187] nut consumption significantly improved FMD (MD $=0.41 \% ; 95 \%$ CI $0.18,0.63 ; p=0.001 ; \mathrm{I}^{2}=39.5 \%$, $p$-het $=0.094)$. Walnuts significantly improved FMD (MD $=0.39 \% ; 95 \% \mathrm{CI} 0.16,0.63 ; p=0.001)$.

Huang et al. 2018 [186] examined the effect nut supplementation on FMD showing a significantly increased FMD (MD = 1.03\%; 95\% CI 0.26, 1.79; $p=0.008$ ) in 13 trials.

Fogacci et al. 2017 [188] investigated the effect of pistachios on endothelial reactivity (ER) by conducting a meta-analysis of 4 RCTs (4 publications) $[173,174,228,229]$. Pistachios did not alter FMD $(\mathrm{MD}=+0.28 \% ; 95 \% \mathrm{CI}-0.58,1.13 ; p=0.525)$.

\subsubsection{Blood Pressure}

Meta-analyses of intervention studies have shown no effect of nut consumption on blood pressure.

A recent meta-analysis of RCTs by Guasch-Ferre et al. 2018 [94] showed that a diet rich in walnuts did not alter systolic blood pressure (SBP) (WMD $=-0.72 \mathrm{~mm} \mathrm{Hg}$; 95\% CI $-2.75,1.30$; $p=0.48)$ or diastolic blood pressure (DBP) (WMD $=-0.10 \mathrm{~mm} \mathrm{Hg} ; 95 \% \mathrm{CI}-1.49,1.30 ; p=0.88)$ [94]. Eight studies from 8 publications (402 subjects) $[95,98,99,102,103,105,107,206]$ were analyzed in this meta-analysis [94].

In a meta-analysis of 21 RCTs (1652 subjects; 21 publications) [95,99,101-103,115,122,125,127, 132,137,139,143,149,191,193,228-232] by Mohammadifard et al. 2015 [181], nut consumption did not significantly alter SBP (MD: $-0.91 ; 95 \% \mathrm{CI}-2.18,0.36 ; \mathrm{P}=0.16$ ) overall but lowered it in subjects without T2DM (MD: $-1.29 \mathrm{mmHg} ; 95 \% \mathrm{CI}-2.35,-0.22 ; p=0.02)$. In sub-analysis stratified by nut types, only pistachios showed a significant reduction in $\mathrm{SBP}(\mathrm{MD}=-1.82 \mathrm{mmHg} ; 95 \% \mathrm{CI}-2.97,-0.67$; $p=0.002$ ), while DBP was significantly reduced by mixed nuts ( $\mathrm{MD}=-1.19 \mathrm{mmHg} ; 95 \% \mathrm{CI}-2.35$, $-0.03 ; p=0.04)$ and pistachios ( $\mathrm{MD}=-0.80 \mathrm{mmHg} ; 95 \% \mathrm{CI}-1.43,-0.17 ; p=0.01$ ) [181]. Mejia et al. 2014 [144] examined the effect of tree nuts on BP (20 studies; $n=1267)$. BP was not significantly changed following tree nut consumption compared with a control diet. Two other meta-analyses $[178,180]$ showed no effect on BP. Gobbo et al. 2015 [180] showed no significant alteration in BP after tree nut consumption (per 1 serving/day) when comparing with a control diet. Mazidi et al. 2016 [178] showed 
no significant changes in SBP (MD $=-0.69 \mathrm{mmHg} ; 95 \% \mathrm{CI}-1.34,-0.03)$ and $\mathrm{DBP}(\mathrm{MD}=-0.14 \mathrm{~mm}$ $\mathrm{Hg}$; 95\% CI $-0.54,0.25)$.

\section{Discussion}

Nuts are comprised of unique macro and micronutrients (e.g., monounsaturated fatty acids, polyunsaturated fatty acids, fiber, arginine and magnesium) and bioactive compounds (e.g., phytosterols and polyphenols). The synergistic beneficial effects of nuts on cardiometabolic disease result from these nutrient- and non-nutrient compositions of nuts. The potential mechanisms underlying these effects can be explained through the improvement of glucose, lipids, weight, endothelial function and gut microbiota modification $[7,233,234]$. The most important and most convincingly demonstrated is the reduction in LDL cholesterol which appears to be similar with all nuts and to be clinically significant.

This review highlights findings from meta-analyses of prospective studies that indicate that nut consumption appears to be associated with reduced all-cause mortality, CVD mortality and incidence, coronary heart disease incidence and mortality stroke incidence and mortality. No association between nut consumption and the incidence of T2DM was found in meta-analyses of prospective studies, even though the fasting glucose levels were reduced by a small degree in intervention studies. Moreover, the association between nut consumption and the incidence of hypertension was found in meta-analyses of prospective studies, whereas blood pressure was not favorably altered in meta-analyses of intervention studies.

In this review we have highlighted differences between meta-analyses and explained why they may differ and come to a conclusion about the likely effects of nuts. No other review has focused entirely on meta-analyses both of all nuts and individual nuts. In general, the more recent meta-analyses are larger and hopefully contain all the previous primary studies in earlier meta-analyses but sometimes this is not the case. Where the meta-analyses are reasonably large the results should be similar even if a few studies are added or omitted. All of the meta-analyses on total mortality and cause-specific mortality assessed the study quality using the Newcastle-Ottawa scale or a very similar scale and found no effect of study quality on the outcomes of the meta-analyses. There appears to be little differences between different nut types.

The major areas of new work required are intervention studies to examine the effects of nuts on glucose homeostasis and the incidence of T2DM in at risk populations as well as clarifying the effects on weight and blood pressure as here there are disparities between cohort studies and interventions.

Supplementary Materials: The following are available online at http:/ /www.mdpi.com/2072-6643/10/12/1935/ s1. Figure S1. Total nuts and all-cause mortality, high vs. low analysis, Figure S2. Total nuts and all-cause mortality, dose-response analysis, Figure S3. Peanuts and all-cause mortality, high vs. low analysis, Figure S4. Tree nuts and all-cause mortality, high vs. low analysis, Figure S5. Total nuts and coronary heart disease mortality, high vs. low analysis, Figure S6. Total nuts and coronary heart disease mortality, dose-response analysis, Figure S7. Total nuts and cardiovascular disease mortality, high vs. low analysis, Figure S8. Total nuts and cardiovascular disease mortality, dose-response analysis, Figure S9. Total nuts and stroke mortality, high vs. low analysis, Figure S10. Total nuts and stroke mortality, dose-response analysis, Figure S11. Total nuts and type 2 diabetes, high vs. low analysis, Figure S12. Total nuts and type 2 diabetes, dose-response analysis, Figure S13. Total nuts and total cardiovascular disease, high vs. low analysis, Figure S14. Total nuts and total cardiovascular disease, dose-response analysis, Figure S15. Total nuts and hypertension, high vs. low analysis, Figure S16. Total nuts and hypertension, dose-response analysis, Figure S17. Total nuts and total coronary heart disease, high vs. low analysis, Figure S18. Total nuts and total coronary heart disease, dose-response analysis, Figure S19. Total nuts and non-fatal/fatal coronary heart disease, dose-response analysis, Figure S20. Total nuts and total stroke, high vs. low analysis, Figure S21. Total nuts and total stroke, dose-response analysis, Figure S22. Nuts and body weight, Figure S23. Nuts and waist circumference, Figure S24. Nuts and body mass index, Figure S25. Nuts and fasting blood glucose, Figure S26. Nuts and total cholesterol, Figure S27. Nuts and low-density lipoprotein cholesterol, Figure S28. Nuts and high-density lipoprotein cholesterol, Figure S29. Nuts and triglycerides, Figure S30. Nuts and apolipoprotein A-1, Figure S31. Nuts and apolipoprotein B, Figure S32. Nuts and apolipoprotein B 100, Figure S33. Nuts and systolic blood pressure, Figure S34. Nuts and diastolic blood pressure, Figure S35. Nuts and adiponectin, Figure S36. Nuts and C-reactive protein (CRP), Figure S37. Nuts and interleukin-6 (IL-6), Figure S38. Nuts and tumor necrosis factor-alpha (TNF- $\alpha$ ), Figure S39. Nuts and intracellular adhesion molecule-1 (ICAM-1), 
Figure S40. Nuts and vascular cell adhesion molecule-1 (VCAM-1), Figure S41. Nuts and E-selectin, and Figure S42. Nuts and flow-mediated dilation (FMD).

Author Contributions: All authors (Y.K., J.K. and P.M.C.) conceived of the manuscript structure and contributed to the writing and editing.

Funding: This research received no external funding.

Acknowledgments: Peter Clifton is supported by a NHMRC Principal Research Fellowship.

Conflicts of Interest: The authors have no conflict of interest related to this manuscript.

\section{References}

1. Guo, F.; Moellering, D.R.; Garvey, W.T. The progression of cardiometabolic disease: Validation of a new cardiometabolic disease staging system applicable to obesity. Obesity 2014, 22, 110-118. [CrossRef] [PubMed]

2. Estruch, R.; Ros, E.; Salas-Salvadó, J.; Covas, M.-I.; Corella, D.; Arós, F.; Gómez-Gracia, E.; Ruiz-Gutiérrez, V.; Fiol, M.; Lapetra, J.; et al. Primary prevention of cardiovascular disease with a mediterranean diet. N. Engl. J. Med. 2013, 368, 1279-1290. [CrossRef] [PubMed]

3. Viguiliouk, E.; Kendall, C.W.; Blanco Mejia, S.; Cozma, A.I.; Ha, V.; Mirrahimi, A.; Jayalath, V.H.; Augustin, L.S.; Chiavaroli, L.; Leiter, L.A.; et al. Effect of tree nuts on glycemic control in diabetes: A systematic review and meta-analysis of randomized controlled dietary trials. PLoS ONE 2014, 9, e103376. [CrossRef] [PubMed]

4. Jaceldo-Siegl, K.; Haddad, E.; Oda, K.; Fraser, G.E.; Sabate, J. Tree nuts are inversely associated with metabolic syndrome and obesity: The adventist health study-2. PLoS ONE 2014, 9, e85133. [CrossRef] [PubMed]

5. Mayhew, A.J.; de Souza, R.J.; Meyre, D.; Anand, S.S.; Mente, A. A systematic review and meta-analysis of nut consumption and incident risk of CVD and all-cause mortality. Br. J. Nutr. 2016, 115, 212-225. [CrossRef] [PubMed]

6. Souza, R.G.; Gomes, A.C.; Naves, M.M.; Mota, J.F. Nuts and legume seeds for cardiovascular risk reduction: Scientific evidence and mechanisms of action. Nutr. Rev. 2015, 73, 335-347. [CrossRef] [PubMed]

7. Kim, Y.; Keogh, J.B.; Clifton, P.M. Benefits of nut consumption on insulin resistance and cardiovascular risk factors: Multiple potential mechanisms of actions. Nutrients 2017, 9, 1271. [CrossRef] [PubMed]

8. Cho, N.H.; Shaw, J.E.; Karuranga, S.; Huang, Y.; da Rocha Fernandes, J.D.; Ohlrogge, A.W.; Malanda, B. IDF diabetes atlas: Global estimates of diabetes prevalence for 2017 and projections for 2045. Diabetes Res. Clin. Pract. 2018, 138, 271-281. [CrossRef] [PubMed]

9. Kirpichnikov, D.; Sowers, J.R. Diabetes mellitus and diabetes-associated vascular disease. Trends Endocrinol. MeTable 2001, 12, 225-230. [CrossRef]

10. McEvoy, C.T.; Cardwell, C.R.; Woodside, J.V.; Young, I.S.; Hunter, S.J.; McKinley, M.C. A posteriori dietary patterns are related to risk of type 2 diabetes: Findings from a systematic review and meta-analysis. J. Acad. Nutr. Diet. 2014, 114, 1759-1775. [CrossRef] [PubMed]

11. Hodge, A.M.; Bassett, J.K.; Dugue, P.A.; Shivappa, N.; Hebert, J.R.; Milne, R.L.; English, D.R.; Giles, G.G. Dietary inflammatory index or mediterranean diet score as risk factors for total and cardiovascular mortality. Nutr. Metab. Cardiovasc. Dis. 2018, 28, 461-469. [CrossRef] [PubMed]

12. Chen, G.C.; Zhang, R.; Martinez-Gonzalez, M.A.; Zhang, Z.L.; Bonaccio, M.; van Dam, R.M.; Qin, L.Q. Nut consumption in relation to all-cause and cause-specific mortality: A meta-analysis 18 prospective studies. Food Funct. 2017, 8, 3893-3905. [CrossRef] [PubMed]

13. Schwingshackl, L.; Schwedhelm, C.; Hoffmann, G.; Lampousi, A.M.; Knuppel, S.; Iqbal, K.; Bechthold, A.; Schlesinger, S.; Boeing, H. Food groups and risk of all-cause mortality: A systematic review and meta-analysis of prospective studies. Am. J. Clin. Nutr. 2017, 105, 1462-1473. [CrossRef] [PubMed]

14. Aune, D.; Keum, N.; Giovannucci, E.; Fadnes, L.T.; Boffetta, P.; Greenwood, D.C.; Tonstad, S.; Vatten, L.J.; Riboli, E.; Norat, T. Nut consumption and risk of cardiovascular disease, total cancer, all-cause and cause-specific mortality: A systematic review and dose-response meta-analysis of prospective studies. BMC Med. 2016, 14, 207. [CrossRef]

15. Grosso, G.; Yang, J.; Marventano, S.; Micek, A.; Galvano, F.; Kales, S.N. Nut consumption on all-cause, cardiovascular and cancer mortality risk: A systematic review and meta-analysis of epidemiologic studies. Am. J. Clin. Nutr. 2015, 101, 783-793. [CrossRef] 
16. Luo, C.; Zhang, Y.; Ding, Y.; Shan, Z.; Chen, S.; Yu, M.; Hu, F.B.; Liu, L. Nut consumption and risk of type 2 diabetes, cardiovascular disease and all-cause mortality: A systematic review and meta-analysis. Am. J. Clin. Nutr. 2014, 100, 256-269. [CrossRef] [PubMed]

17. van den Brandt, P.A.; Schouten, L.J. Relationship of tree nut, peanut and peanut butter intake with total and cause-specific mortality: A cohort study and meta-analysis. Int. J. Epidemiol. 2015, 44, 1038-1049. [CrossRef] [PubMed]

18. Luu, H.N.; Blot, W.J.; Xiang, Y.B.; Cai, H.; Hargreaves, M.K.; Li, H.; Yang, G.; Signorello, L.; Gao, Y.T.; Zheng, W.; et al. Prospective evaluation of the association of nut/peanut consumption with total and cause-specific mortality. JAMA Intern. Med. 2015, 175, 755-766. [CrossRef]

19. Bao, Y.; Han, J.; Hu, F.B.; Giovannucci, E.L.; Stampfer, M.J.; Willett, W.C.; Fuchs, C.S. Association of nut consumption with total and cause-specific mortality. N. Engl. J. Med. 2013, 369, 2001-2011. [CrossRef]

20. Fraser, G.E.; Shavlik, D.J. Risk factors for all-cause and coronary heart disease mortality in the oldest-old. The adventist health study. Arch. Intern. Med. 1997, 157, 2249-2258. [CrossRef]

21. Hshieh, T.T.; Petrone, A.B.; Gaziano, J.M.; Djousse, L. Nut consumption and risk of mortality in the physicians' health study. Am. J. Clin. Nutr. 2015, 101, 407-412. [CrossRef] [PubMed]

22. Blomhoff, R.; Carlsen, M.H.; Andersen, L.F.; Jacobs, D.R., Jr. Health benefits of nuts: Potential role of antioxidants. Br. J. Nutr. 2006, 96 (Suppl. 2), S52-S60. [CrossRef] [PubMed]

23. Fernandez-Montero, A.; Bes-Rastrollo, M.; Barrio-Lopez, M.T.; Fuente-Arrillaga Cde, L.; Salas-Salvado, J.; Moreno-Galarraga, L.; Martinez-Gonzalez, M.A. Nut consumption and 5-y all-cause mortality in a mediterranean cohort: The sun project. Nutrition 2014, 30, 1022-1027. [CrossRef] [PubMed]

24. Fraser, G.E.; Sumbureru, D.; Pribis, P.; Neil, R.L.; Frankson, M.A. Association among health habits, risk factors and all-cause mortality in a black california population. Epidemiology 1997, 8, 168-174. [CrossRef] [PubMed]

25. Guasch-Ferre, M.; Bullo, M.; Martinez-Gonzalez, M.A.; Ros, E.; Corella, D.; Estruch, R.; Fito, M.; Aros, F.; Warnberg, J.; Fiol, M.; et al. Frequency of nut consumption and mortality risk in the predimed nutrition intervention trial. BMC Med. 2013, 11, 164. [CrossRef]

26. Mann, J.I.; Appleby, P.N.; Key, T.J.; Thorogood, M. Dietary determinants of ischaemic heart disease in health conscious individuals. Heart 1997, 78, 450-455. [CrossRef]

27. Bonaccio, M.; Di Castelnuovo, A.; De Curtis, A.; Costanzo, S.; Bracone, F.; Persichillo, M.; Donati, M.B.; de Gaetano, G.; Iacoviello, L. Nut consumption is inversely associated with both cancer and total mortality in a mediterranean population: Prospective results from the moli-sani study. Br. J. Nutr. 2015, 114, 804-811. [CrossRef]

28. Gopinath, B.; Flood, V.M.; Burlutksy, G.; Mitchell, P. Consumption of nuts and risk of total and cause-specific mortality over 15 years. Nutr. Metab. Cardiovasc. Dis. 2015, 25, 1125-1131. [CrossRef]

29. Eslamparast, T.; Sharafkhah, M.; Poustchi, H.; Hashemian, M.; Dawsey, S.M.; Freedman, N.D.; Boffetta, P.; Abnet, C.C.; Etemadi, A.; Pourshams, A.; et al. Nut consumption and total and cause-specific mortality: Results from the golestan cohort study. Int. J. Epidemiol. 2017, 46, 75-85. [CrossRef]

30. Sluik, D.; Boeing, H.; Li, K.; Kaaks, R.; Johnsen, N.F.; Tjonneland, A.; Arriola, L.; Barricarte, A.; Masala, G.; Grioni, S.; et al. Lifestyle factors and mortality risk in individuals with diabetes mellitus: Are the associations different from those in individuals without diabetes? Diabetologia 2014, 57, 63-72. [CrossRef]

31. Wang, J.B.; Fan, J.H.; Dawsey, S.M.; Sinha, R.; Freedman, N.D.; Taylor, P.R.; Qiao, Y.L.; Abnet, C.C. Dietary components and risk of total, cancer and cardiovascular disease mortality in the linxian nutrition intervention trials cohort in china. Sci. Rep. 2016, 6, 22619. [CrossRef] [PubMed]

32. Leenders, M.; Sluijs, I.; Ros, M.M.; Boshuizen, H.C.; Siersema, P.D.; Ferrari, P.; Weikert, C.; Tjonneland, A.; Olsen, A.; Boutron-Ruault, M.C.; et al. Fruit and vegetable consumption and mortality: European prospective investigation into cancer and nutrition. Am. J. Epidemiol. 2013, 178, 590-602. [CrossRef] [PubMed]

33. di Giuseppe, R.; Fjeld, M.K.; Dierkes, J.; Theoflylaktopoulou, D.; Arregui, M.; Boeing, H.; Weikert, C. The association between nut consumption and the risk of total and ischemic stroke in a german cohort study. Eur. J. Clin. Nutr. 2015, 69, 431-435. [CrossRef] [PubMed]

34. Yochum, L.A.; Folsom, A.R.; Kushi, L.H. Intake of antioxidant vitamins and risk of death from stroke in postmenopausal women. Am. J. Clin. Nutr. 2000, 72, 476-483. [CrossRef] [PubMed] 
35. Fitzgerald, K.C.; Chiuve, S.E.; Buring, J.E.; Ridker, P.M.; Glynn, R.J. Comparison of associations of adherence to a dietary approaches to stop hypertension (dash)-style diet with risks of cardiovascular disease and venous thromboembolism. J. Thromb. Haemost. 2012, 10, 189-198. [CrossRef] [PubMed]

36. von Ruesten, A.; Feller, S.; Bergmann, M.M.; Boeing, H. Diet and risk of chronic diseases: Results from the first 8 years of follow-up in the epic-potsdam study. Eur. J. Clin. Nutr. 2013, 67, 412-419. [CrossRef]

37. Haring, B.; Gronroos, N.; Nettleton, J.A.; von Ballmoos, M.C.; Selvin, E.; Alonso, A. Dietary protein intake and coronary heart disease in a large community based cohort: Results from the atherosclerosis risk in communities (aric) study [corrected]. PLoS ONE 2014, 9, e109552. [CrossRef]

38. Albert, C.M.; Gaziano, J.M.; Willett, W.C.; Manson, J.E. Nut consumption and decreased risk of sudden cardiac death in the physicians' health study. Arch. Intern. Med. 2002, 162, 1382-1387. [CrossRef]

39. Fraser, G.E.; Sabate, J.; Beeson, W.L.; Strahan, T.M. A possible protective effect of nut consumption on risk of coronary heart disease. The adventist health study. Arch. Intern. Med. 1992, 152, 1416-1424. [CrossRef]

40. Bernstein, A.M.; Sun, Q.; Hu, F.B.; Stampfer, M.J.; Manson, J.E.; Willett, W.C. Major dietary protein sources and risk of coronary heart disease in women. Circulation 2010, 122, 876-883. [CrossRef]

41. Haring, B.; Misialek, J.R.; Rebholz, C.M.; Petruski-Ivleva, N.; Gottesman, R.F.; Mosley, T.H.; Alonso, A. Association of dietary protein consumption with incident silent cerebral infarcts and stroke: The atherosclerosis risk in communities (aric) study. Stroke 2015, 46, 3443-3450. [CrossRef] [PubMed]

42. Bernstein, A.M.; Pan, A.; Rexrode, K.M.; Stampfer, M.; Hu, F.B.; Mozaffarian, D.; Willett, W.C. Dietary protein sources and the risk of stroke in men and women. Stroke 2012, 43, 637-644. [CrossRef] [PubMed]

43. Djousse, L.; Gaziano, J.M.; Kase, C.S.; Kurth, T. Nut consumption and risk of stroke in US male physicians. Clin. Nutr. 2010, 29, 605-609. [CrossRef] [PubMed]

44. Yaemsiri, S.; Sen, S.; Tinker, L.; Rosamond, W.; Wassertheil-Smoller, S.; He, K. Trans fat, aspirin and ischemic stroke in postmenopausal women. Ann. Neurol. 2012, 72, 704-715. [CrossRef]

45. Chiuve, S.E.; Cook, N.R.; Shay, C.M.; Rexrode, K.M.; Albert, C.M.; Manson, J.E.; Willett, W.C.; Rimm, E.B. Lifestyle-based prediction model for the prevention of CVD: The healthy heart score. J. Am. Heart Assoc. 2014, 3, e000954. [CrossRef] [PubMed]

46. Gopinath, B.; Buyken, A.E.; Flood, V.M.; Empson, M.; Rochtchina, E.; Mitchell, P. Consumption of polyunsaturated fatty acids, fish and nuts and risk of inflammatory disease mortality. Am. J. Clin. Nutr. 2011, 93, 1073-1079. [CrossRef] [PubMed]

47. Hu, F.B.; Stampfer, M.J.; Manson, J.E.; Rimm, E.B.; Colditz, G.A.; Rosner, B.A.; Speizer, F.E.; Hennekens, C.H.; Willett, W.C. Frequent nut consumption and risk of coronary heart disease in women: Prospective cohort study. BMJ 1998, 317, 1341-1345. [CrossRef]

48. Li, T.Y.; Brennan, A.M.; Wedick, N.M.; Mantzoros, C.; Rifai, N.; Hu, F.B. Regular consumption of nuts is associated with a lower risk of cardiovascular disease in women with type 2 diabetes. J. Nutr. 2009, 139, 1333-1338. [CrossRef]

49. Levitan, E.B.; Lewis, C.E.; Tinker, L.F.; Eaton, C.B.; Ahmed, A.; Manson, J.E.; Snetselaar, L.G.; Martin, L.W.; Trevisan, M.; Howard, B.V.; et al. Mediterranean and dash diet scores and mortality in women with heart failure: The women's health initiative. Circ. Heart Fail. 2013, 6, 1116-1123. [CrossRef]

50. van den Brandt, P.A. The impact of a mediterranean diet and healthy lifestyle on premature mortality in men and women. Am. J. Clin. Nutr. 2011, 94, 913-920. [CrossRef]

51. Ellsworth, J.L.; Kushi, L.H.; Folsom, A.R. Frequent nut intake and risk of death from coronary heart disease and all causes in postmenopausal women: The iowa women's health study. Nutr. Metab. Cardiovasc. Dis. 2001, 11, 372-377. [PubMed]

52. Djousse, L.; Petrone, A.; Gaziano, J. Abstract p067: Nut consumption is associated with a lower risk of death among US male physicians. Circulation 2014, 129, AP067.

53. Kochar, J.; Gaziano, J.M.; Djousse, L. Nut consumption and risk of type ii diabetes in the physicians' health study. Eur. J. Clin. Nutr. 2010, 64, 75-79. [CrossRef] [PubMed]

54. Parker, E.D.; Harnack, L.J.; Folsom, A.R. Nut consumption and risk of type 2 diabetes. Jama 2003, 290, 38-39. [CrossRef] [PubMed]

55. Pan, A.; Sun, Q.; Manson, J.E.; Willett, W.C.; Hu, F.B. Walnut consumption is associated with lower risk of type 2 diabetes in women. J. Nutr. 2013, 143, 512-518. [CrossRef] [PubMed] 
56. Villegas, R.; Gao, Y.T.; Yang, G.; Li, H.L.; Elasy, T.A.; Zheng, W.; Shu, X.O. Legume and soy food intake and the incidence of type 2 diabetes in the shanghai women's health study. Am. J. Clin. Nutr. 2008, 87, 162-167. [CrossRef] [PubMed]

57. Afshin, A.; Micha, R.; Khatibzadeh, S.; Mozaffarian, D. Consumption of nuts and legumes and risk of incident ischemic heart disease, stroke and diabetes: A systematic review and meta-analysis. Am. J. Clin. Nutr. 2014, 100, 278-288. [CrossRef]

58. Weng, Y.Q.; Yao, J.; Guo, M.L.; Qin, Q.J.; Li, P. Association between nut consumption and coronary heart disease: A meta-analysis. Coron. Artery Dis. 2016, 27, 227-232. [CrossRef]

59. Wu, L.; Wang, Z.; Zhu, J.; Murad, A.L.; Prokop, L.J.; Murad, M.H. Nut consumption and risk of cancer and type 2 diabetes: A systematic review and meta-analysis. Nutr. Rev. 2015, 73, 409-425. [CrossRef]

60. Schwingshackl, L.; Hoffmann, G.; Lampousi, A.M.; Knuppel, S.; Iqbal, K.; Schwedhelm, C.; Bechthold, A.; Schlesinger, S.; Boeing, H. Food groups and risk of type 2 diabetes mellitus: A systematic review and meta-analysis of prospective studies. Eur. J. Epidemiol. 2017, 32, 363-375. [CrossRef]

61. Asghari, G.; Ghorbani, Z.; Mirmiran, P.; Azizi, F. Nut consumption is associated with lower incidence of type 2 diabetes: The tehran lipid and glucose study. Diabetes MeTable 2017, 43, 18-24. [CrossRef] [PubMed]

62. Buijsse, B.; Boeing, H.; Drogan, D.; Schulze, M.B.; Feskens, E.J.; Amiano, P.; Barricarte, A.; Clavel-Chapelon, F.; de Lauzon-Guillain, B.; Fagherazzi, G.; et al. Consumption of fatty foods and incident type 2 diabetes in populations from eight european countries. Eur. J. Clin. Nutr. 2015, 69, 455-461. [CrossRef] [PubMed]

63. Guo, K.; Zhou, Z.; Jiang, Y.; Li, W.; Li, Y. Meta-analysis of prospective studies on the effects of nut consumption on hypertension and type 2 diabetes mellitus. J. Diabetes 2015, 7, 202-212. [CrossRef] [PubMed]

64. Zhou, D.; Yu, H.; He, F.; Reilly, K.H.; Zhang, J.; Li, S.; Zhang, T.; Wang, B.; Ding, Y.; Xi, B. Nut consumption in relation to cardiovascular disease risk and type 2 diabetes: A systematic review and meta-analysis of prospective studies. Am. J. Clin. Nutr. 2014, 100, 270-277. [CrossRef] [PubMed]

65. Montonen, J.; Jarvinen, R.; Heliovaara, M.; Reunanen, A.; Aromaa, A.; Knekt, P. Food consumption and the incidence of type ii diabetes mellitus. Eur. J. Clin. Nutr. 2005, 59, 441-448. [CrossRef] [PubMed]

66. Salas-Salvado, J.; Bullo, M.; Estruch, R.; Ros, E.; Covas, M.I.; Ibarrola-Jurado, N.; Corella, D.; Aros, F.; Gomez-Gracia, E.; Ruiz-Gutierrez, V.; et al. Prevention of diabetes with mediterranean diets: A subgroup analysis of a randomized trial. Ann. Intern. Med. 2014, 160, 1-10. [CrossRef] [PubMed]

67. Schwingshackl, L.; Schwedhelm, C.; Hoffmann, G.; Knuppel, S.; Iqbal, K.; Andriolo, V.; Bechthold, A.; Schlesinger, S.; Boeing, H. Food groups and risk of hypertension: A systematic review and dose-response meta-analysis of prospective studies. Adv. Nutr. 2017, 8, 793-803. [CrossRef] [PubMed]

68. Djousse, L.; Rudich, T.; Gaziano, J.M. Nut consumption and risk of hypertension in US male physicians. Clin. Nutr. 2009, 28, 10-14. [CrossRef]

69. Golzarand, M.; Bahadoran, Z.; Mirmiran, P.; Azizi, F. Protein foods group and 3-year incidence of hypertension: A prospective study from tehran lipid and glucose study. J. Ren. Nutr. 2016, 26, 219-225. [CrossRef]

70. Martinez-Lapiscina, E.H.; Pimenta, A.M.; Beunza, J.J.; Bes-Rastrollo, M.; Martinez, J.A.; Martinez-Gonzalez, M.A. Nut consumption and incidence of hypertension: The sun prospective cohort. Nutr. Metab. Cardiovasc. Dis. 2010, 20, 359-365. [CrossRef]

71. Weng, L.C.; Steffen, L.M.; Szklo, M.; Nettleton, J.; Chambless, L.; Folsom, A.R. A diet pattern with more dairy and nuts but less meat is related to lower risk of developing hypertension in middle-aged adults: The atherosclerosis risk in communities (aric) study. Nutrients 2013, 5, 1719-1733. [CrossRef]

72. Steffen, L.M.; Kroenke, C.H.; Yu, X.; Pereira, M.A.; Slattery, M.L.; Van Horn, L.; Gross, M.D.; Jacobs, D.R., Jr. Associations of plant food, dairy product and meat intakes with 15-y incidence of elevated blood pressure in young black and white adults: The coronary artery risk development in young adults (cardia) study. Am. J. Clin. Nutr. 2005, 82, 1169-1177. [CrossRef] [PubMed]

73. Bechthold, A.; Boeing, H.; Schwedhelm, C.; Hoffmann, G.; Knuppel, S.; Iqbal, K.; De Henauw, S.; Michels, N.; Devleesschauwer, B.; Schlesinger, S.; et al. Food groups and risk of coronary heart disease, stroke and heart failure: A systematic review and dose-response meta-analysis of prospective studies. Crit. Rev. Food Sci. Nutr. 2017, 17, 1-17. [CrossRef] [PubMed]

74. Ma, L.; Wang, F.; Guo, W.; Yang, H.; Liu, Y.; Zhang, W. Nut consumption and the risk of coronary artery disease: A dose-response meta-analysis of 13 prospective studies. Thromb. Res. 2014, 134, 790-794. [CrossRef] [PubMed] 
75. Shi, Z.Q.; Tang, J.J.; Wu, H.; Xie, C.Y.; He, Z.Z. Consumption of nuts and legumes and risk of stroke: A meta-analysis of prospective cohort studies. Nutr. Metab. Cardiovasc. Dis. 2014, 24, 1262-1271. [CrossRef] [PubMed]

76. Bernstein, A.M.; de Koning, L.; Flint, A.J.; Rexrode, K.M.; Willett, W.C. Soda consumption and the risk of stroke in men and women. Am. J. Clin. Nutr. 2012, 95, 1190-1199. [CrossRef] [PubMed]

77. Li, H.; Li, X.; Yuan, S.; Jin, Y.; Lu, J. Nut consumption and risk of metabolic syndrome and overweight/obesity: A meta-analysis of prospective cohort studies and randomized trials. Nutr. MeTable 2018, 15, 46. [CrossRef] [PubMed]

78. Lutsey, P.L.; Steffen, L.M.; Stevens, J. Dietary intake and the development of the metabolic syndrome: The atherosclerosis risk in communities study. Circulation 2008, 117, 754-761. [CrossRef] [PubMed]

79. Fernandez-Montero, A.; Bes-Rastrollo, M.; Beunza, J.J.; Barrio-Lopez, M.T.; de la Fuente-Arrillaga, C.; Moreno-Galarraga, L.; Martinez-Gonzalez, M.A. Nut consumption and incidence of metabolic syndrome after 6-year follow-up: The sun (seguimiento universidad de navarra, university of navarra follow-up) cohort. Public Health Nutr. 2013, 16, 2064-2072. [CrossRef] [PubMed]

80. Hosseinpour-Niazi, S.; Hosseini, S.; Mirmiran, P.; Azizi, F. Prospective study of nut consumption and incidence of metabolic syndrome: Tehran lipid and glucose study. Nutrients 2017, 9, 1056. [CrossRef] [PubMed]

81. Bes-Rastrollo, M.; Sabate, J.; Gomez-Gracia, E.; Alonso, A.; Martinez, J.A.; Martinez-Gonzalez, M.A. Nut consumption and weight gain in a mediterranean cohort: The sun study. Obesity 2007, 15, 107-116. [CrossRef]

82. Freisling, H.; Noh, H.; Slimani, N.; Chajes, V.; May, A.M.; Peeters, P.H.; Weiderpass, E.; Cross, A.J.; Skeie, G.; Jenab, M.; et al. Nut intake and 5-year changes in body weight and obesity risk in adults: Results from the epic-panacea study. Eur. J. Nutr. 2018, 57, 2399-2408. [CrossRef] [PubMed]

83. Bes-Rastrollo, M.; Wedick, N.M.; Martinez-Gonzalez, M.A.; Li, T.Y.; Sampson, L.; Hu, F.B. Prospective study of nut consumption, long-term weight change and obesity risk in women. Am. J. Clin. Nutr. 2009, 89, 1913-1919. [CrossRef] [PubMed]

84. Nettleton, J.A.; Hivert, M.F.; Lemaitre, R.N.; McKeown, N.M.; Mozaffarian, D.; Tanaka, T.; Wojczynski, M.K.; Hruby, A.; Djousse, L.; Ngwa, J.S.; et al. Meta-analysis investigating associations between healthy diet and fasting glucose and insulin levels and modification by loci associated with glucose homeostasis in data from 15 cohorts. Am. J. Epidemiol. 2013, 177, 103-115. [CrossRef]

85. Ezzati, M.; Hoorn, S.V.; Rodgers, A.; Lopez, A.D.; Mathers, C.D.; Murray, C.J. Estimates of global and regional potential health gains from reducing multiple major risk factors. Lancet 2003, 362, 271-280. [CrossRef]

86. Jiang, R.; Manson, J.E.; Stampfer, M.J.; Liu, S.; Willett, W.C.; Hu, F.B. Nut and peanut butter consumption and risk of type 2 diabetes in women. Jama 2002, 288, 2554-2560. [CrossRef] [PubMed]

87. Key, T.J.; Thorogood, M.; Appleby, P.N.; Burr, M.L. Dietary habits and mortality in 11,000 vegetarians and health conscious people: Results of a 17 year follow up. BMJ 1996, 313, 775-779. [CrossRef] [PubMed]

88. Trichopoulou, A.; Bamia, C.; Trichopoulos, D. Mediterranean diet and survival among patients with coronary heart disease in Greece. Arch. Intern. Med. 2005, 165, 929-935. [CrossRef]

89. Dilis, V.; Katsoulis, M.; Lagiou, P.; Trichopoulos, D.; Naska, A.; Trichopoulou, A. Mediterranean diet and chd: The greek European prospective investigation into cancer and nutrition cohort. Br. J. Nutr. 2012, 108, 699-709. [CrossRef] [PubMed]

90. Fraser, G.E.; Lindsted, K.D.; Beeson, W.L. Effect of risk factor values on lifetime risk of and age at first coronary event. The adventist health study. Am. J. Epidemiol. 1995, 142, 746-758. [CrossRef] [PubMed]

91. Urpi-Sarda, M.; Casas, R.; Chiva-Blanch, G.; Romero-Mamani, E.S.; Valderas-Martinez, P.; Salas-Salvado, J.; Covas, M.I.; Toledo, E.; Andres-Lacueva, C.; Llorach, R.; et al. The mediterranean diet pattern and its main components are associated with lower plasma concentrations of tumor necrosis factor receptor 60 in patients at high risk for cardiovascular disease. J. Nutr. 2012, 142, 1019-1025. [CrossRef] [PubMed]

92. Kushi, L.H.; Folsom, A.R.; Prineas, R.J.; Mink, P.J.; Wu, Y.; Bostick, R.M. Dietary antioxidant vitamins and death from coronary heart disease in postmenopausal women. N. Engl. J. Med. 1996, 334, 1156-1162. [CrossRef] [PubMed]

93. Prineas, R.J.; Kushi, L.H.; Folsom, A.R.; Bostick, R.M.; Wu, Y. Walnuts and serum lipids. N. Engl. J. Med. $1993,329,359$. 
94. Guasch-Ferre, M.; Li, J.; Hu, F.B.; Salas-Salvado, J.; Tobias, D.K. Effects of walnut consumption on blood lipids and other cardiovascular risk factors: An updated meta-analysis and systematic review of controlled trials. Am. J. Clin. Nutr. 2018, 108, 174-187. [CrossRef] [PubMed]

95. Ma, Y.; Njike, V.Y.; Millet, J.; Dutta, S.; Doughty, K.; Treu, J.A.; Katz, D.L. Effects of walnut consumption on endothelial function in type 2 diabetic subjects: A randomized controlled crossover trial. Diabetes Care 2010, 33, 227-232. [CrossRef] [PubMed]

96. Tapsell, L.C.; Gillen, L.J.; Patch, C.S.; Batterham, M.; Owen, A.; Bare, M.; Kennedy, M. Including walnuts in a low-fat/modified-fat diet improves hdl cholesterol-to-total cholesterol ratios in patients with type 2 diabetes. Diabetes Care 2004, 27, 2777-2783. [CrossRef]

97. Almario, R.U.; Vonghavaravat, V.; Wong, R.; Kasim-Karakas, S.E. Effects of walnut consumption on plasma fatty acids and lipoproteins in combined hyperlipidemia. Am. J. Clin. Nutr. 2001, 74, 72-79. [CrossRef]

98. Damasceno, N.R.; Perez-Heras, A.; Serra, M.; Cofan, M.; Sala-Vila, A.; Salas-Salvado, J.; Ros, E. Crossover study of diets enriched with virgin olive oil, walnuts or almonds. Effects on lipids and other cardiovascular risk markers. Nutr. Metab. Cardiovasc. Dis. 2011, 21 (Suppl. 1), S14-S20. [CrossRef]

99. Wu, H.; Pan, A.; Yu, Z.; Qi, Q.; Lu, L.; Zhang, G.; Yu, D.; Zong, G.; Zhou, Y.; Chen, X.; et al. Lifestyle counseling and supplementation with flaxseed or walnuts influence the management of metabolic syndrome. J. Nutr. 2010, 140, 1937-1942. [CrossRef]

100. Tapsell, L.C.; Batterham, M.J.; Teuss, G.; Tan, S.Y.; Dalton, S.; Quick, C.J.; Gillen, L.J.; Charlton, K.E. Long-term effects of increased dietary polyunsaturated fat from walnuts on metabolic parameters in type ii diabetes. Eur. J. Clin. Nutr. 2009, 63, 1008-1015. [CrossRef]

101. Spaccarotella, K.J.; Kris-Etherton, P.M.; Stone, W.L.; Bagshaw, D.M.; Fishell, V.K.; West, S.G.; Lawrence, F.R.; Hartman, T.J. The effect of walnut intake on factors related to prostate and vascular health in older men. Nutr. J. 2008, 7, 13. [CrossRef] [PubMed]

102. Mukuddem-Petersen, J.; Stonehouse Oosthuizen, W.; Jerling, J.C.; Hanekom, S.M.; White, Z. Effects of a high walnut and high cashew nut diet on selected markers of the metabolic syndrome: A controlled feeding trial. Br. J. Nutr. 2007, 97, 1144-1153. [CrossRef]

103. Ros, E.; Núñez, I.; Pérez-Heras, A.; Serra, M.; Gilabert, R.; Casals, E.; Deulofeu, R. A walnut diet improves endothelial function in hypercholesterolemic subjects: A randomized crossover trial. Circulation 2004, 109, 1609. [CrossRef]

104. Zambon, D.; Sabate, J.; Munoz, S.; Campero, B.; Casals, E.; Merlos, M.; Laguna, J.C.; Ros, E. Substituting walnuts for monounsaturated fat improves the serum lipid profile of hypercholesterolemic men and women. A randomized crossover trial. Ann. Intern. Med. 2000, 132, 538-546. [CrossRef] [PubMed]

105. Katz, D.L.; Davidhi, A.; Ma, Y.; Kavak, Y.; Bifulco, L.; Njike, V.Y. Effects of walnuts on endothelial function in overweight adults with visceral obesity: A randomized, controlled, crossover trial. J. Am. Coll. Nutr. 2012, 31, 415-423. [CrossRef]

106. Canales, A.; Sanchez-Muniz, F.J.; Bastida, S.; Librelotto, J.; Nus, M.; Corella, D.; Guillen, M.; Benedi, J. Effect of walnut-enriched meat on the relationship between VCAM, ICAM and LTB4 levels and PON-1 activity in ApoA4 360 and PON-1 allele carriers at increased cardiovascular risk. Eur. J. Clin. Nutr. 2011, 65, 703-710. [CrossRef] [PubMed]

107. Schutte, A.E.; Van Rooyen, J.M.; Huisman, H.W.; Mukuddem-Petersen, J.; Oosthuizen, W.; Hanekom, S.M.; Jerling, J.C. Modulation of baroreflex sensitivity by walnuts versus cashew nuts in subjects with metabolic syndrome. Am. J. Hypertens. 2006, 19, 629-636. [CrossRef]

108. Perna, S.; Giacosa, A.; Bonitta, G.; Bologna, C.; Isu, A.; Guido, D.; Rondanelli, M. Effects of hazelnut consumption on blood lipids and body weight: A systematic review and bayesian meta-analysis. Nutrients 2016, 8, 747. [CrossRef] [PubMed]

109. Tey, S.L.; Brown, R.; Gray, A.; Chisholm, A.; Delahunty, C. Nuts improve diet quality compared to other energy-dense snacks while maintaining body weight. J. Nutr. MeTable 2011, 2011, 357350. [CrossRef]

110. Tey, S.L.; Gray, A.R.; Chisholm, A.W.; Delahunty, C.M.; Brown, R.C. The dose of hazelnuts influences acceptance and diet quality but not inflammatory markers and body composition in overweight and obese individuals. J. Nutr. 2013, 143, 1254. [CrossRef] [PubMed]

111. Orem, A.; Yucesan, F.B.; Orem, C.; Akcan, B.; Kural, B.V.; Alasalvar, C.; Shahidi, F. Hazelnut-enriched diet improves cardiovascular risk biomarkers beyond a lipid-lowering effect in hypercholesterolemic subjects. J. Clin. Lipidol. 2013, 7, 123-131. [CrossRef] [PubMed] 
112. Banel, D.K.; Hu, F.B. Effects of walnut consumption on blood lipids and other cardiovascular risk factors: A meta-analysis and systematic review. Am. J. Clin. Nutr. 2009, 90, 56-63. [CrossRef] [PubMed]

113. Zhao, G.; Etherton, T.D.; Martin, K.R.; Gillies, P.J.; West, S.G.; Kris-Etherton, P.M. Dietary alpha-linolenic acid inhibits proinflammatory cytokine production by peripheral blood mononuclear cells in hypercholesterolemic subjects. Am. J. Clin. Nutr. 2007, 85, 385-391. [CrossRef] [PubMed]

114. Canales, A.; Benedi, J.; Nus, M.; Librelotto, J.; Sanchez-Montero, J.M.; Sanchez-Muniz, F.J. Effect of walnut-enriched restructured meat in the antioxidant status of overweight/obese senior subjects with at least one extra chd-risk factor. J. Am. Coll. Nutr. 2007, 26, 225-232. [CrossRef] [PubMed]

115. Iwamoto, M.; Imaizumi, K.; Sato, M.; Hirooka, Y.; Sakai, K.; Takeshita, A.; Kono, M. Serum lipid profiles in Japanese women and men during consumption of walnuts. Eur. J. Clin. Nutr. 2002, 56, 629-637. [CrossRef] [PubMed]

116. Morgan, J.M.; Horton, K.; Reese, D.; Carey, C.; Walker, K.; Capuzzi, D.M. Effects of walnut consumption as part of a low-fat, low-cholesterol diet on serum cardiovascular risk factors. Int. J. Vitam. Nutr. Res. 2002, 72, 341-347. [CrossRef] [PubMed]

117. Perez-Martinez, P.; Lopez-Miranda, J.; Blanco-Colio, L.; Bellido, C.; Jimenez, Y.; Moreno, J.A.; Delgado-Lista, J.; Egido, J.; Perez-Jimenez, F. The chronic intake of a mediterranean diet enriched in virgin olive oil, decreases nuclear transcription factor $\mathrm{kb}$ activation in peripheral blood mononuclear cells from healthy men. Atherosclerosis 2007, 194, e141-e146. [CrossRef]

118. Sabate, J.; Fraser, G.E.; Burke, K.; Knutsen, S.F.; Bennett, H.; Lindsted, K.D. Effects of walnuts on serum lipid levels and blood pressure in normal men. N. Engl. J. Med. 1993, 328, 603-607. [CrossRef]

119. Chisholm, A.; Mann, J.; Skeaff, M.; Frampton, C.; Sutherland, W.; Duncan, A.; Tiszavari, S. A diet rich in walnuts favourably influences plasma fatty acid profile in moderately hyperlipidaemic subjects. Eur. J. Clin. Nutr. 1998, 52, 12-16. [CrossRef]

120. Flores-Mateo, G.; Rojas-Rueda, D.; Basora, J.; Ros, E.; Salas-Salvadó, J. Nut intake and adiposity: Meta-analysis of clinical trials. Am. J. Clin. Nutr. 2013, 97, 1346-1355. [CrossRef]

121. O'Byrne, D.J.; Knauft, D.A.; Shireman, R.B. Low fat-monounsaturated rich diets containing high-oleic peanuts improve serum lipoprotein profiles. Lipids 1997, 32, 687-695. [CrossRef] [PubMed]

122. Jenkins, D.J.; Kendall, C.W.; Marchie, A.; Parker, T.L.; Connelly, P.W.; Qian, W.; Haight, J.S.; Faulkner, D.; Vidgen, E.; Lapsley, K.G.; et al. Dose response of almonds on coronary heart disease risk factors: Blood lipids, oxidized low-density lipoproteins, lipoprotein(a), homocysteine and pulmonary nitric oxide: A randomized, controlled, crossover trial. Circulation 2002, 106, 1327-1332. [CrossRef] [PubMed]

123. Jenkins, D.J.; Kendall, C.W.; Marchie, A.; Faulkner, D.; Vidgen, E.; Lapsley, K.G.; Trautwein, E.A.; Parker, T.L.; Josse, R.G.; Leiter, L.A.; et al. The effect of combining plant sterols, soy protein, viscous fibers and almonds in treating hypercholesterolemia. Metabolism 2003, 52, 1478-1483. [CrossRef]

124. Jenkins, D.J.; Popovich, D.G.; Kendall, C.W.; Vidgen, E.; Tariq, N.; Ransom, T.P.; Wolever, T.M.; Vuksan, V.; Mehling, C.C.; Boctor, D.L.; et al. Effect of a diet high in vegetables, fruit and nuts on serum lipids. Metabolism 1997, 46, 530-537. [CrossRef]

125. Wien, M.A.; Sabate, J.M.; Ikle, D.N.; Cole, S.E.; Kandeel, F.R. Almonds vs complex carbohydrates in a weight reduction program. Int. J. Obes. Relat. Metab. Disord. 2003, 27, 1365-1372. [CrossRef]

126. Hollis, J.; Mattes, R. Effect of chronic consumption of almonds on body weight in healthy humans. Br. J. Nutr. 2007, 98, 651-656. [CrossRef]

127. Sheridan, M.J.; Cooper, J.N.; Erario, M.; Cheifetz, C.E. Pistachio nut consumption and serum lipid levels. J. Am. Coll. Nutr. 2007, 26, 141-148. [CrossRef]

128. Olmedilla-Alonso, B.; Granado-Lorencio, F.; Herrero-Barbudo, C.; Blanco-Navarro, I.; Blazquez-Garcia, S.; Perez-Sacristan, B. Consumption of restructured meat products with added walnuts has a cholesterol-lowering effect in subjects at high cardiovascular risk: A randomised, crossover, placebo-controlled study. J. Am. Coll. Nutr. 2008, 27, 342-348. [CrossRef]

129. Gebauer, S.K.; West, S.G.; Kay, C.D.; Alaupovic, P.; Bagshaw, D.; Kris-Etherton, P.M. Effects of pistachios on cardiovascular disease risk factors and potential mechanisms of action: A dose-response study. Am. J. Clin. Nutr. 2008, 88, 651-659. [CrossRef]

130. Tapsell, L.; Batterham, M.; Tan, S.Y.; Warensjo, E. The effect of a calorie controlled diet containing walnuts on substrate oxidation during 8-h in a room calorimeter. J. Am. Coll. Nutr. 2009, 28, 611-617. [CrossRef] 
131. Razquin, C.; Martinez, J.A.; Martinez-Gonzalez, M.A.; Fernandez-Crehuet, J.; Santos, J.M.; Marti, A. A mediterranean diet rich in virgin olive oil may reverse the effects of the $-174 \mathrm{~g} / \mathrm{c}$ il6 gene variant on 3-year body weight change. Mol. Nutr. Food Res. 2010, 54 (Suppl. 1), S75-S82. [CrossRef] [PubMed]

132. Casas-Agustench, P.; López-Uriarte, P.; Bulló, M.; Ros, E.; Cabré-Vila, J.; Salas-Salvadó, J. Effects of one serving of mixed nuts on serum lipids, insulin resistance and inflammatory markers in patients with the metabolic syndrome. Nutr. Metab. Cardiovasc. Dis. 2011, 21, 126-135. [CrossRef]

133. Sabate, J.; Haddad, E.; Tanzman, J.S.; Jambazian, P.; Rajaram, S. Serum lipid response to the graduated enrichment of a step i diet with almonds: A randomized feeding trial. Am. J. Clin. Nutr. 2003, 77, 1379-1384. [CrossRef] [PubMed]

134. Spiller, G.A.; Jenkins, D.A.; Bosello, O.; Gates, J.E.; Cragen, L.N.; Bruce, B. Nuts and plasma lipids: An almond-based diet lowers LDL-C while preserving HDL-C. J. Am. Coll. Nutr. 1998, 17, 285-290. [CrossRef] [PubMed]

135. Lamarche, B.; Desroches, S.; Jenkins, D.J.; Kendall, C.W.; Marchie, A.; Faulkner, D.; Vidgen, E.; Lapsley, K.G.; Trautwein, E.A.; Parker, T.L.; et al. Combined effects of a dietary portfolio of plant sterols, vegetable protein, viscous fibre and almonds on LDL particle size. Br. J. Nutr. 2004, 92, 657-663. [CrossRef] [PubMed]

136. Sabate, J.; Cordero-Macintyre, Z.; Siapco, G.; Torabian, S.; Haddad, E. Does regular walnut consumption lead to weight gain? Br. J. Nutr. 2005, 94, 859-864. [CrossRef]

137. Wien, M.; Bleich, D.; Raghuwanshi, M.; Gould-Forgerite, S.; Gomes, J.; Monahan-Couch, L.; Oda, K. Almond consumption and cardiovascular risk factors in adults with prediabetes. J. Am. Coll. Nutr. 2010, 29, 189-197. [CrossRef]

138. Chisholm, A.; Mc Auley, K.; Mann, J.; Williams, S.; Skeaff, M. Cholesterol lowering effects of nuts compared with a canola oil enriched cereal of similar fat composition. Nutr. Metab. Cardiovasc. Dis. 2005, 15, 284-292. [CrossRef]

139. Foster, G.D.; Shantz, K.L.; Vander Veur, S.S.; Oliver, T.L.; Lent, M.R.; Virus, A.; Szapary, P.O.; Rader, D.J.; Zemel, B.S.; Gilden-Tsai, A. A randomized trial of the effects of an almond-enriched, hypocaloric diet in the treatment of obesity. Am. J. Clin. Nutr. 2012, 96, 249-254. [CrossRef]

140. Mercanligil, S.M.; Arslan, P.; Alasalvar, C.; Okut, E.; Akgul, E.; Pinar, A.; Geyik, P.O.; Tokgozoglu, L.; Shahidi, F. Effects of hazelnut-enriched diet on plasma cholesterol and lipoprotein profiles in hypercholesterolemic adult men. Eur. J. Clin. Nutr. 2007, 61, 212-220. [CrossRef]

141. Morgan, W.A.; Clayshulte, B.J. Pecans lower low-density lipoprotein cholesterol in people with normal lipid levels. J. Am. Diet. Assoc. 2000, 100, 312-318. [CrossRef]

142. Kocyigit, A.; Koylu, A.A.; Keles, H. Effects of pistachio nuts consumption on plasma lipid profile and oxidative status in healthy volunteers. Nutr. Metab. Cardiovasc. Dis. 2006, 16, 202-209. [CrossRef] [PubMed]

143. Li, S.C.; Liu, Y.H.; Liu, J.F.; Chang, W.H.; Chen, C.M.; Chen, C.Y. Almond consumption improved glycemic control and lipid profiles in patients with type 2 diabetes mellitus. Metabolism 2011, 60, 474-479. [CrossRef] [PubMed]

144. Blanco Mejia, S.; Kendall, C.W.; Viguiliouk, E.; Augustin, L.S.; Ha, V.; Cozma, A.I.; Mirrahimi, A.; Maroleanu, A.; Chiavaroli, L.; Leiter, L.A.; et al. Effect of tree nuts on metabolic syndrome criteria: A systematic review and meta-analysis of randomised controlled trials. BMJ Open 2014, 4, e004660. [CrossRef] [PubMed]

145. Wang, X.; Li, Z.; Liu, Y.; Lv, X.; Yang, W. Effects of pistachios on body weight in chinese subjects with metabolic syndrome. Nutr. J. 2012, 11, 20. [CrossRef] [PubMed]

146. Tan, S.Y.; Mattes, R.D. Appetitive, dietary and health effects of almonds consumed with meals or as snacks: A randomized, controlled trial. Eur. J. Clin. Nutr. 2013, 67, 1205-1214. [CrossRef] [PubMed]

147. Gulati, S.; Misra, A.; Pandey, R.M.; Bhatt, S.P.; Saluja, S. Effects of pistachio nuts on body composition, metabolic, inflammatory and oxidative stress parameters in asian indians with metabolic syndrome: A 24-wk, randomized control trial. Nutrition 2014, 30, 192-197. [CrossRef]

148. Somerset, S.M.; Graham, L.; Markwell, K. Isoenergetic replacement of dietary saturated with monounsaturated fat via macadamia nuts enhances endothelial function in overweight subjects. e-SPEN J. 2013, 8, e113-e119. [CrossRef]

149. Jenkins, D.J.; Kendall, C.W.; Banach, M.S.; Srichaikul, K.; Vidgen, E.; Mitchell, S.; Parker, T.; Nishi, S.; Bashyam, B.; de Souza, R.; et al. Nuts as a replacement for carbohydrates in the diabetic diet. Diabetes Care 2011, 34, 1706-1711. [CrossRef] 
150. Damavandi, R.D. The effects of cashew consumption on serum glucose, insulin and lipoprotein in type 2 diabetic patients. Iran. J. Endocrinol. MeTable 2012, 14, 325-334.

151. Lee, Y.J.; Nam, G.E.; Seo, J.A.; Yoon, T.; Seo, I.; Lee, J.H.; Im, D.; Bahn, K.N.; Jeong, S.A.; Kang, T.S.; et al. Nut consumption has favorable effects on lipid profiles of Korean women with metabolic syndrome. Nutr. Res. 2014, 34, 814-820. [CrossRef] [PubMed]

152. Alves, R.D.M.; Moreira, A.P.B.; Macedo, V.S.; Alfenas, R.; Bressan, J.; Mattes, R.; Costa, N.M.B. Regular intake of high-oleic peanuts improves fat oxidation and body composition in overweight/obese men pursuing a energy-restricted diet. Obesity 2014, 22, 1422-1429. [CrossRef] [PubMed]

153. Wien, M.; Oda, K.; Sabate, J. A randomized controlled trial to evaluate the effect of incorporating peanuts into an american diabetes association meal plan on the nutrient profile of the total diet and cardiometabolic parameters of adults with type 2 diabetes. Nutr. J. 2014, 13, 10. [CrossRef] [PubMed]

154. Barbour, J.A.; Howe, P.R.; Buckley, J.D.; Bryan, J.; Coates, A.M. Effect of 12 weeks high oleic peanut consumption on cardio-metabolic risk factors and body composition. Nutrients 2015, 7, 7381-7398. [CrossRef]

155. Jaceldo-Siegl, K.; Sabate, J.; Batech, M.; Fraser, G.E. Influence of body mass index and serum lipids on the cholesterol-lowering effects of almonds in free-living individuals. Nutr. Metab. Cardiovasc. Dis. 2011, 21 (Suppl. 1), S7-S13. [CrossRef] [PubMed]

156. Abazarfard, Z.; Salehi, M.; Keshavarzi, S. The effect of almonds on anthropometric measurements and lipid profile in overweight and obese females in a weight reduction program: A randomized controlled clinical trial. J. Res. Med. Sci. 2014, 19, 457-464. [PubMed]

157. Babio, N.; Toledo, E.; Estruch, R.; Ros, E.; Martinez-Gonzalez, M.A.; Castaner, O.; Bullo, M.; Corella, D.; Aros, F.; Gomez-Gracia, E.; et al. Mediterranean diets and metabolic syndrome status in the predimed randomized trial. Can. Med. Assoc. J. 2014, 186, E649-E657. [CrossRef] [PubMed]

158. Bento, A.P.; Cominetti, C.; Simoes Filho, A.; Naves, M.M. Baru almond improves lipid profile in mildly hypercholesterolemic subjects: A randomized, controlled, crossover study. Nutr. Metab. Cardiovasc. Dis. 2014, 24, 1330-1336. [CrossRef]

159. Berryman, C.E.; West, S.G.; Fleming, J.A.; Bordi, P.L.; Kris-Etherton, P.M. Effects of daily almond consumption on cardiometabolic risk and abdominal adiposity in healthy adults with elevated LDL-cholesterol: A randomized controlled trial. J. Am. Heart Assoc. 2015, 4, e000993. [CrossRef]

160. Jamshed, H.; Sultan, F.A.; Iqbal, R.; Gilani, A.H. Dietary almonds increase serum HDL cholesterol in coronary artery disease patients in a randomized controlled trial. J. Nutr. 2015, 145, 2287-2292. [CrossRef]

161. Dhillon, J.; Tan, S.Y.; Mattes, R.D. Almond consumption during energy restriction lowers truncal fat and blood pressure in compliant overweight or obese adults. J. Nutr. 2016, 146, 2513-2519. [CrossRef] [PubMed]

162. Ruisinger, J.F.; Gibson, C.A.; Backes, J.M.; Smith, B.K.; Sullivan, D.K.; Moriarty, P.M.; Kris-Etherton, P. Statins and almonds to lower lipoproteins (the stall study). J. Clin. Lipidol. 2015, 9, 58-64. [CrossRef]

163. Chen, C.M.; Liu, J.F.; Li, S.C.; Huang, C.L.; Hsirh, A.T.; Weng, S.F.; Chang, M.L.; Li, H.T.; Mohn, E.; Chen, C.O. Almonds ameliorate glycemic control in Chinese patients with better controlled type 2 diabetes: A randomized, crossover, controlled feeding trial. Nutr. MeTable 2017, 14, 51. [CrossRef] [PubMed]

164. Jung, H.; Chen, C.O.; Blumberg, J.B.; Kwak, H.K. The effect of almonds on vitamin e status and cardiovascular risk factors in korean adults: A randomized clinical trial. Eur. J. Nutr. 2018, 57, 2069-2079. [CrossRef] [PubMed]

165. Liu, Y.; Hwang, H.J.; Ryu, H.; Lee, Y.S.; Kim, H.S.; Park, H. The effects of daily intake timing of almond on the body composition and blood lipid profile of healthy adults. Nutr. Res. Pract. 2017, 11, 479-486. [CrossRef] [PubMed]

166. Le, T.; Flatt, S.W.; Natarajan, L.; Pakiz, B.; Quintana, E.L.; Heath, D.D.; Rana, B.K.; Rock, C.L. Effects of diet composition and insulin resistance status on plasma lipid levels in a weight loss intervention in women. J. Am. Heart Assoc. 2016, 5, e002771. [CrossRef] [PubMed]

167. Rock, C.L.; Flatt, S.W.; Pakiz, B.; Quintana, E.L.; Heath, D.D.; Rana, B.K.; Natarajan, L. Effects of diet composition on weight loss, metabolic factors and biomarkers in a 1-year weight loss intervention in obese women examined by baseline insulin resistance status. Metabolism 2016, 65, 1605-1613. [CrossRef] [PubMed]

168. Neale, E.P.; Tapsell, L.C.; Martin, A.; Batterham, M.J.; Wibisono, C.; Probst, Y.C. Impact of providing walnut samples in a lifestyle intervention for weight loss: A secondary analysis of the healthtrack trial. Food Nutr. Res. 2017, 61, 1344522. [CrossRef] 
169. Rock, C.L.; Flatt, S.W.; Barkai, H.S.; Pakiz, B.; Heath, D.D. Walnut consumption in a weight reduction intervention: Effects on body weight, biological measures, blood pressure and satiety. Nutr. J. 2017, 16, 76. [CrossRef]

170. Tapsell, L.C.; Lonergan, M.; Batterham, M.J.; Neale, E.P.; Martin, A.; Thorne, R.; Deane, F.; Peoples, G. Effect of interdisciplinary care on weight loss: A randomised controlled trial. BMJ Open 2017, 7, e014533. [CrossRef]

171. Li, Z.; Song, R.; Nguyen, C.; Zerlin, A.; Karp, H.; Naowamondhol, K.; Thames, G.; Gao, K.; Li, L.; Tseng, C.H.; et al. Pistachio nuts reduce triglycerides and body weight by comparison to refined carbohydrate snack in obese subjects on a 12-week weight loss program. J. Am. Coll. Nutr. 2010, 29, 198-203. [CrossRef] [PubMed]

172. Hernandez-Alonso, P.; Salas-Salvado, J.; Baldrich-Mora, M.; Juanola-Falgarona, M.; Bullo, M. Beneficial effect of pistachio consumption on glucose metabolism, insulin resistance, inflammation and related metabolic risk markers: A randomized clinical trial. Diabetes Care 2014, 37, 3098-3105. [CrossRef] [PubMed]

173. Kasliwal, R.R.; Bansal, M.; Mehrotra, R.; Yeptho, K.P.; Trehan, N. Effect of pistachio nut consumption on endothelial function and arterial stiffness. Nutrition 2015, 31, 678-685. [CrossRef] [PubMed]

174. Sauder, K.A.; McCrea, C.E.; Ulbrecht, J.S.; Kris-Etherton, P.M.; West, S.G. Effects of pistachios on the lipid/lipoprotein profile, glycemic control, inflammation and endothelial function in type 2 diabetes: A randomized trial. Metabolism 2015, 64, 1521-1529. [CrossRef]

175. Damavandi, R.D.; Eghtesadi, S.; Shidfar, F.; Heydari, I.; Foroushani, A.R. Effects of hazelnuts consumption on fasting blood sugar and lipoproteins in patients with type 2 diabetes. J. Res. Med. Sci. 2013, 18, 314-321.

176. Njike, V.Y.; Ayettey, R.; Petraro, P.; Treu, J.A.; Katz, D.L. Walnut ingestion in adults at risk for diabetes: Effects on body composition, diet quality and cardiac risk measures. BMJ Open Diabetes Res. Care 2015, 3, e000115. [CrossRef]

177. Parham, M.; Heidari, S.; Khorramirad, A.; Hozoori, M.; Hosseinzadeh, F.; Bakhtyari, L.; Vafaeimanesh, J. Effects of pistachio nut supplementation on blood glucose in patients with type 2 diabetes: A randomized crossover trial. Rev. Diab. Stud. 2014, 11, 190-196. [CrossRef]

178. Mazidi, M.; Rezaie, P.; Ferns, G.A.; Gao, H.K. Impact of different types of tree nut, peanut and soy nut consumption on serum c-reactive protein (CRP): A systematic review and meta-analysis of randomized controlled clinical trials. Medicine 2016, 95, e5165. [CrossRef]

179. Musa-Veloso, K.; Paulionis, L.; Poon, T.; Lee, H.Y. The effects of almond consumption on fasting blood lipid levels: A systematic review and meta-analysis of randomised controlled trials. J. Nutr. Sci. 2016, 5, e34. [CrossRef]

180. Del Gobbo, L.C.; Falk, M.C.; Feldman, R.; Lewis, K.; Mozaffarian, D. Effects of tree nuts on blood lipids, apolipoproteins and blood pressure: Systematic review, meta-analysis and dose-response of 61 controlled intervention trials. Am. J. Clin. Nutr. 2015, 102, 1347-1356. [CrossRef]

181. Mohammadifard, N.; Salehi-Abargouei, A.; Salas-Salvado, J.; Guasch-Ferre, M.; Humphries, K.; Sarrafzadegan, N. The effect of tree nut, peanut and soy nut consumption on blood pressure: A systematic review and meta-analysis of randomized controlled clinical trials. Am. J. Clin. Nutr. 2015, 101, 966-982. [CrossRef] [PubMed]

182. Sabate, J.; Oda, K.; Ros, E. Nut consumption and blood lipid levels: A pooled analysis of 25 intervention trials. Arch. Intern. Med. 2010, 170, 821-827. [CrossRef] [PubMed]

183. Phung, O.J.; Makanji, S.S.; White, C.M.; Coleman, C.I. Almonds have a neutral effect on serum lipid profiles: A meta-analysis of randomized trials. J. Am. Diet. Assoc. 2009, 109, 865-873. [CrossRef] [PubMed]

184. Xiao, Y.; Xia, J.; Ke, Y.; Cheng, J.; Yuan, J.; Wu, S.; Lv, Z.; Huang, S.; Kim, J.H.; Wong, S.Y.; et al. Effects of nut consumption on selected inflammatory markers: A systematic review and meta-analysis of randomized controlled trials. Nutrition 2018, 54, 129-143. [CrossRef] [PubMed]

185. Neale, E.P.; Tapsell, L.C.; Guan, V.; Batterham, M.J. The effect of nut consumption on markers of inflammation and endothelial function: A systematic review and meta-analysis of randomised controlled trials. BMJ Open 2017, 7, e016863. [CrossRef] [PubMed]

186. Huang, Y.; Zheng, S.; Wang, T.; Yang, X.; Luo, Q.; Li, H. Effect of oral nut supplementation on endothelium-dependent vasodilation-A meta-analysis. VASA Z. Gefasskrankh. 2018, 47, 203-207. [CrossRef] [PubMed] 
187. Xiao, Y.; Huang, W.; Peng, C.; Zhang, J.; Wong, C.; Kim, J.H.; Yeoh, E.K.; Su, X. Effect of nut consumption on vascular endothelial function: A systematic review and meta-analysis of randomized controlled trials. Clin. Nutr. 2018, 37, 831-839. [CrossRef]

188. Fogacci, F.; Cicero, A.F.G.; Derosa, G.; Rizzo, M.; Veronesi, M.; Borghi, C. Effect of pistachio on brachial artery diameter and flow-mediated dilatation: A systematic review and meta-analysis of randomized, controlled-feeding clinical studies. Crit. Rev. Food Sci. Nutr. 2017. [CrossRef]

189. Colpo, E.; Dalton, D.A.V.C.; Reetz, L.G.; Duarte, M.M.; Farias, I.L.; Meinerz, D.F.; Mariano, D.O.; Vendrusculo, R.G.; Boligon, A.A.; Dalla Corte, C.L.; et al. Brazilian nut consumption by healthy volunteers improves inflammatory parameters. Nutrition 2014, 30, 459-465. [CrossRef]

190. Rajaram, S.; Connell, K.M.; Sabate, J. Effect of almond-enriched high-monounsaturated fat diet on selected markers of inflammation: A randomised, controlled, crossover study. Br. J. Nutr. 2010, 103, 907-912. [CrossRef]

191. Bakhtiary, A.; Yassin, Z.; Hanachi, P.; Rahmat, A.; Ahmad, Z.; Jalali, F. Effects of soy on metabolic biomarkers of cardiovascular disease in elderly women with metabolic syndrome. Arch. Iran. Med. 2012, 15, 462-468. [PubMed]

192. Reverri, E.J.; LaSalle, C.D.; Franke, A.A.; Steinberg, F.M. Soy provides modest benefits on endothelial function without affecting inflammatory biomarkers in adults at cardiometabolic risk. Mol. Nutr. Food Res. 2015, 59, 323-333. [CrossRef] [PubMed]

193. Azadbakht, L.; Kimiagar, M.; Mehrabi, Y.; Esmaillzadeh, A.; Padyab, M.; Hu, F.B.; Willett, W.C. Soy inclusion in the diet improves features of the metabolic syndrome: A randomized crossover study in postmenopausal women. Am. J. Clin. Nutr. 2007, 85, 735-741. [CrossRef] [PubMed]

194. Brennan, A.M.; Sweeney, L.L.; Liu, X.; Mantzoros, C.S. Walnut consumption increases satiation but has no effect on insulin resistance or the metabolic profile over a 4-day period. Obesity 2010, 18, 1176-1182. [CrossRef] [PubMed]

195. Aronis, K.N.; Vamvini, M.T.; Chamberland, J.P.; Sweeney, L.L.; Brennan, A.M.; Magkos, F.; Mantzoros, C.S. Short-term walnut consumption increases circulating total adiponectin and apolipoprotein a concentrations but does not affect markers of inflammation or vascular injury in obese humans with the metabolic syndrome: Data from a double-blinded, randomized, placebo-controlled study. Metabolism 2012, 61, 577-582. [PubMed]

196. Wu, L.; Piotrowski, K.; Rau, T.; Waldmann, E.; Broedl, U.C.; Demmelmair, H.; Koletzko, B.; Stark, R.G.; Nagel, J.M.; Mantzoros, C.S.; et al. Walnut-enriched diet reduces fasting non-hdl-cholesterol and apolipoprotein $\mathrm{b}$ in healthy caucasian subjects: A randomized controlled cross-over clinical trial. Metabolism 2014, 63, 382-391. [CrossRef] [PubMed]

197. Moreira Alves, R.D.; Boroni Moreira, A.P.; Macedo, V.S.; Bressan, J.; de Cassia Goncalves Alfenas, R.; Mattes, R.; Brunoro Costa, N.M. High-oleic peanuts: New perspective to attenuate glucose homeostasis disruption and inflammation related obesity. Obesity 2014, 22, 1981-1988. [CrossRef] [PubMed]

198. Jenkins, D.J.; Kendall, C.W.; Marchie, A.; Josse, A.R.; Nguyen, T.H.; Faulkner, D.A.; Lapsley, K.G.; Blumberg, J. Almonds reduce biomarkers of lipid peroxidation in older hyperlipidemic subjects. J. Nutr. 2008, 138, 908-913. [CrossRef]

199. Holligan, S.; West, S.; Gebauer, S.; Kay, C.; Kris-Etherton, P. A moderate-fat diet with pistachios lowers small-dense LDL and improves markers of insulin sensitivity in subjects with moderately-elevated cholesterol levels. Faseb J. 2013, 27, 1057.

200. Anderson, A.; Anderson, M.; Jacobson, J.; Popko, M.; Young, J.; Limburg, P.; Wilson, T. Metabolic effects of bedtime pistachio consumption for 6 weeks in overweight persons. Faseb J. 2013, 27, 1072.

201. Sauder, K.; Mccrea, C.; Kris-Etherton, P.; Ulbrecht, J.; West, S. Effect of pistachios on lipids, lipoproteins, glucose metabolism and insulin sensitivity in type 2 diabetes. Faseb J. 2013, 27, 368.

202. Lovejoy, J.C.; Most, M.M.; Lefevre, M.; Greenway, F.L.; Rood, J.C. Effect of diets enriched in almonds on insulin action and serum lipids in adults with normal glucose tolerance or type 2 diabetes. Am. J. Clin. Nutr. 2002, 76, 1000-1006. [CrossRef] [PubMed]

203. Cohen, A.E.; Johnston, C.S. Almond ingestion at mealtime reduces postprandial glycemia and chronic ingestion reduces hemoglobin a(1c) in individuals with well-controlled type 2 diabetes mellitus. Metabolism 2011, 60, 1312-1317. [CrossRef] [PubMed] 
204. Bamberger, C.; Rossmeier, A.; Lechner, K.; Wu, L.; Waldmann, E.; Stark, R.G.; Altenhofer, J.; Henze, K.; Parhofer, K.G. A walnut-enriched diet reduces lipids in healthy caucasian subjects, independent of recommended macronutrient replacement and time point of consumption: A prospective, randomized, controlled trial. Nutrients 2017, 9, 1097. [CrossRef]

205. Burns-Whitmore, B.; Haddad, E.; Sabate, J.; Rajaram, S. Effects of supplementing n-3 fatty acid enriched eggs and walnuts on cardiovascular disease risk markers in healthy free-living lacto-ovo-vegetarians: A randomized, crossover, free-living intervention study. Nutr. J. 2014, 13, 29. [CrossRef] [PubMed]

206. Din, J.N.; Aftab, S.M.; Jubb, A.W.; Carnegy, F.H.; Lyall, K.; Sarma, J.; Newby, D.E.; Flapan, A.D. Effect of moderate walnut consumption on lipid profile, arterial stiffness and platelet activation in humans. Eur. J. Clin. Nutr. 2011, 65, 234-239. [CrossRef] [PubMed]

207. Perez-Martinez, P.; Ordovas, J.M.; Garcia-Rios, A.; Delgado-Lista, J.; Delgado-Casado, N.; Cruz-Teno, C.; Camargo, A.; Yubero-Serrano, E.M.; Rodriguez, F.; Perez-Jimenez, F.; et al. Consumption of diets with different type of fat influences triacylglycerols-rich lipoproteins particle number and size during the postprandial state. Nutr. Metab. Cardiovasc. Dis. 2011, 21, 39-45. [CrossRef] [PubMed]

208. Torabian, S.; Haddad, E.; Cordero-MacIntyre, Z.; Tanzman, J.; Fernandez, M.L.; Sabate, J. Long-term walnut supplementation without dietary advice induces favorable serum lipid changes in free-living individuals. Eur. J. Clin. Nutr. 2010, 64, 274-279. [CrossRef]

209. Rajaram, S.; Haddad, E.H.; Mejia, A.; Sabaté, J. Walnuts and fatty fish influence different serum lipid fractions in normal to mildly hyperlipidemic individuals: A randomized controlled study. Am. J. Clin. Nutr. 2009, 89, 1657S. [CrossRef]

210. Zhao, G.; Etherton, T.D.; Martin, K.R.; West, S.G.; Gillies, P.J.; Kris-Etherton, P.M. Dietary alpha-linolenic acid reduces inflammatory and lipid cardiovascular risk factors in hypercholesterolemic men and women. J. Nutr. 2004, 134, 2991-2997. [CrossRef] [PubMed]

211. Sweazea, K.L.; Johnston, C.S.; Ricklefs, K.D.; Petersen, K.N. Almond supplementation in the absence of dietary advice significantly reduces c-reactive protein in subjects with type 2 diabetes. J. Funct. Foods 2014, 10, 252-259. [CrossRef]

212. Kurlandsky, S.B.; Stote, K.S. Cardioprotective effects of chocolate and almond consumption in healthy women. Nutr. Res. 2006, 26, 509-516. [CrossRef]

213. Jia, X.; Li, N.; Zhang, W.; Zhang, X.; Lapsley, K.; Huang, G.; Blumberg, J.; Ma, G.; Chen, J. A pilot study on the effects of almond consumption on DNA damage and oxidative stress in smokers. Nutr. Cancer 2006, 54, 179-183. [CrossRef] [PubMed]

214. Tamizifar, B.; Rismankarzadeh, M.; Vosoughi, A.-A.; Rafieeyan, M.; Tamizifar, B.; Aminzade, A. A low-dose almond-based diet decreases LDL-C while preserving HDL-C. Arch. Iran. Med. 2005, 8, 45-51.

215. Rajaram, S.; Burke, K.; Connell, B.; Myint, T.; Sabate, J. A monounsaturated fatty acid-rich pecan-enriched diet favorably alters the serum lipid profile of healthy men and women. J. Nutr. 2001, 131, 2275-2279. [CrossRef] [PubMed]

216. Curb, J.D.; Wergowske, G.; Dobbs, J.C.; Abbott, R.D.; Huang, B. Serum lipid effects of a high-monounsaturated fat diet based on macadamia nuts. Arch. Intern. Med. 2000, 160, 1154-1158. [CrossRef]

217. Griel, A.E.; Cao, Y.; Bagshaw, D.D.; Cifelli, A.M.; Holub, B.; Kris-Etherton, P.M. A macadamia nut-rich diet reduces total and LDL-cholesterol in mildly hypercholesterolemic men and women. J. Nutr. 2008, 138, 761-767. [CrossRef]

218. Lopez-Uriarte, P.; Nogues, R.; Saez, G.; Bullo, M.; Romeu, M.; Masana, L.; Tormos, C.; Casas-Agustench, P.; Salas-Salvado, J. Effect of nut consumption on oxidative stress and the endothelial function in metabolic syndrome. Clin. Nutr. 2010, 29, 373-380. [CrossRef]

219. Chen, C.Y.; Holbrook, M.; Duess, M.A.; Dohadwala, M.M.; Hamburg, N.M.; Asztalos, B.F.; Milbury, P.E.; Blumberg, J.B.; Vita, J.A. Effect of almond consumption on vascular function in patients with coronary artery disease: A randomized, controlled, cross-over trial. Nutr. J. 2015, 14, 61. [CrossRef]

220. Chiang, Y.L.; Haddad, E.; Rajaram, S.; Shavlik, D.; Sabate, J. The effect of dietary walnuts compared to fatty fish on eicosanoids, cytokines, soluble endothelial adhesion molecules and lymphocyte subsets: A randomized, controlled crossover trial. Prostaglandins Leukot. Essent. Fatty Acids 2012, 87, 111-117. [CrossRef]

221. Djoussé, L.; Lu, B.; Gaziano, J. Effects of walnut consumption on endothelial function in people with type 2 diabetes: A randomized pilot trial. Curr. Nutr. Rep. 2016, 5, 1-8. [CrossRef] 
222. Hu, Y.; McIntosh, G.H.; Le Leu, R.K.; Somashekar, R.; Meng, X.Q.; Gopalsamy, G.; Bambaca, L.; McKinnon, R.A.; Young, G.P. Supplementation with brazil nuts and green tea extract regulates targeted biomarkers related to colorectal cancer risk in humans. Br. J. Nutr. 2016, 116, 1901-1911. [CrossRef] [PubMed]

223. Liu, J.F.; Liu, Y.H.; Chen, C.M.; Chang, W.H.; Chen, C.Y. The effect of almonds on inflammation and oxidative stress in Chinese patients with type 2 diabetes mellitus: A randomized crossover controlled feeding trial. Eur. J. Nutr. 2013, 52, 927-935. [CrossRef]

224. Casas, R.; Sacanella, E.; Urpi-Sarda, M.; Chiva-Blanch, G.; Ros, E.; Martinez-Gonzalez, M.A.; Covas, M.I.; Salas-Salvado, J.; Fiol, M.; Aros, F.; et al. The effects of the mediterranean diet on biomarkers of vascular wall inflammation and plaque vulnerability in subjects with high risk for cardiovascular disease. A randomized trial. PLoS ONE 2014, 9, e100084. [CrossRef] [PubMed]

225. Casas, R.; Sacanella, E.; Urpi-Sarda, M.; Corella, D.; Castaner, O.; Lamuela-Raventos, R.M.; Salas-Salvado, J.; Martinez-Gonzalez, M.A.; Ros, E.; Estruch, R. Long-term immunomodulatory effects of a mediterranean diet in adults at high risk of cardiovascular disease in the prevencion con dieta mediterranea (predimed) randomized controlled trial. J. Nutr. 2016, 146, 1684-1693. [CrossRef] [PubMed]

226. Lasa, A.; Miranda, J.; Bullo, M.; Casas, R.; Salas-Salvado, J.; Larretxi, I.; Estruch, R.; Ruiz-Gutiérrez, V.; Portillo, M. Comparative effect of two mediterranean diets versus a low-fat diet on glycaemic control in individuals with type 2 diabetes. Eur. J. Clin. Nutr. 2014, 68, 767-772. [CrossRef] [PubMed]

227. Sola, R.; Valls, R.M.; Godas, G.; Perez-Busquets, G.; Ribalta, J.; Girona, J.; Heras, M.; Cabre, A.; Castro, A.; Domenech, G.; et al. Cocoa, hazelnuts, sterols and soluble fiber cream reduces lipids and inflammation biomarkers in hypertensive patients: A randomized controlled trial. PLoS ONE 2012, 7, e31103. [CrossRef]

228. West, S.G.; Gebauer, S.K.; Kay, C.D.; Bagshaw, D.M.; Savastano, D.M.; Diefenbach, C.; Kris-Etherton, P.M. Diets containing pistachios reduce systolic blood pressure and peripheral vascular responses to stress in adults with dyslipidemia. Hypertension 2012, 60, 58-63. [CrossRef] [PubMed]

229. Sari, I.; Baltaci, Y.; Bagci, C.; Davutoglu, V.; Erel, O.; Celik, H.; Ozer, O.; Aksoy, N.; Aksoy, M. Effect of pistachio diet on lipid parameters, endothelial function, inflammation and oxidative status: A prospective study. Nutrition 2010, 26, 399-404. [CrossRef]

230. Ghadimi Nouran, M.; Kimiagar, M.; Abadi, A.; Mirzazadeh, M.; Harrison, G. Peanut consumption and cardiovascular risk. Public Health Nutr. 2010, 13, 1581-1586. [CrossRef]

231. Claesson, A.L.; Holm, G.; Ernersson, A.; Lindstrom, T.; Nystrom, F.H. Two weeks of overfeeding with candy but not peanuts, increases insulin levels and body weight. Scand. J. Clin. Lab. Investig. 2009, 69, 598-605. [CrossRef] [PubMed]

232. Estruch, R.; Martinez-Gonzalez, M.A.; Corella, D.; Salas-Salvado, J.; Ruiz-Gutierrez, V.; Covas, M.I.; Fiol, M.; Gomez-Gracia, E.; Lopez-Sabater, M.C.; Vinyoles, E.; et al. Effects of a mediterranean-style diet on cardiovascular risk factors: A randomized trial. Ann. Intern. Med. 2006, 145, 1-11. [CrossRef] [PubMed]

233. Coates, A.M.; Howe, P.R. Edible nuts and metabolic health. Curr. Opin. Lipidol. 2007, 18, 25-30. [CrossRef] [PubMed]

234. Hernandez-Alonso, P.; Camacho-Barcia, L.; Bullo, M.; Salas-Salvado, J. Nuts and dried fruits: An update of their beneficial effects on type 2 diabetes. Nutrients 2017, 9, 673. [CrossRef]

(C) 2018 by the authors. Licensee MDPI, Basel, Switzerland. This article is an open access article distributed under the terms and conditions of the Creative Commons Attribution (CC BY) license (http://creativecommons.org/licenses/by/4.0/). 